\title{
Functional Coupling of TRPM2 and NMDARs exacerbates excitotoxicity in ischemic brain injury
}

Pengyu Zong ${ }^{1}$, Jianlin Feng ${ }^{1}$, Zhichao Yue ${ }^{1}$, Gongxiong Wu², Baonan Sun ${ }^{1}$, Yanlin He${ }^{1}$, Barbara Miller ${ }^{3}$,

Albert S. Yu, Zhongping Su${ }^{1}$, Yasuo Mori" ${ }^{4}$ Jia Xie ${ }^{1}$, Lixia Yue ${ }^{1, *}$

${ }^{1}$ Department of Cell Biology, Calhoun Cardiology Center, University of Connecticut School of Medicine (UConn Health), Farmington, CT 06030, USA;

2 Department of Medicine, Brigham and Women's Hospital, Laboratory for Translational Research, Harvard Medical School, Cambridge, MA 02139, USA;

${ }^{3}$ Departments of Biochemistry and Molecular Biology, The Pennsylvania State University College of Medicine, P.O. Box 850, Hershey, Pennsylvania, 17033, USA;

${ }^{4}$ Laboratory of Molecular Biology, Department of Synthetic Chemistry and Biological Chemistry, Graduate School of Engineering, Kyoto University, Kyoto, 615-8510, Japan; The World Premier International Research InitiativeInstitute for Integrated Cell-Material Sciences, Kyoto University, Kyoto, 615-8510, Japan. Electronic

*Corresponding author: Lixia Yue: lyue@uchc.edu 


\section{SUMMARY}

Excitotoxicity caused by NMDA receptors (NMDARs) is a major cause of neuronal death in ischemic stroke. However, past efforts of directly targeting NMDARs have unfortunately failed in clinical ischemic stroke trials. Here we reveal an unexpected mechanism underlying NMDARs-mediated neurotoxicity, which leads to identification of a novel target and development of an effective therapeutic peptide for ischemic stroke. We show that NMDAR's excitotoxicity upon ischemic insults is mediated by physical and functional coupling to TRPM2. The physical interaction of TRPM2 with NMDARs results in markedly increase in the surface expression of NMDARs, leading to enhanced NMDAR function and increased neuronal death. We identified a specific NMDAR-interacting domain on TRPM2, and developed a cell-permeable peptide to uncouple TRPM2-NMDARs. The disrupting-peptide protects neurons against ischemic injury in vitro and protects mice against ischemic stroke in vivo. These findings provide an unconventional strategy to eliminate excitotoxic neuronal death without directly targeting NMDARs.

\section{KEY WORDS}

Ischemic stroke, TRPM2, NMDA receptors (NMDARs), $\mathrm{Ca}^{2+}$ signaling, excitotoxicity, neuronal death, therapeutic peptide 


\section{HIGHLIGHTS}

- $\quad$ TRPM2 physically and functionally interacts with NMDARs

- Interaction of TRPM2 with NMDARs exacerbates NMDAR's extrasynaptic excitotoxicity by increasing NMDAR's surface expression during ischemic injury

- TRPM2 recruits PKC $\gamma$ to the interacting complexes to increase NMDAR's surface expression

- Uncoupling the interaction between TRPM2 and NMDARs with a disrupting peptide (TAT-EE 3 ) protects neurons against ischemic stroke in vitro and in vivo

\section{GRAPHIC ABSTRACT}
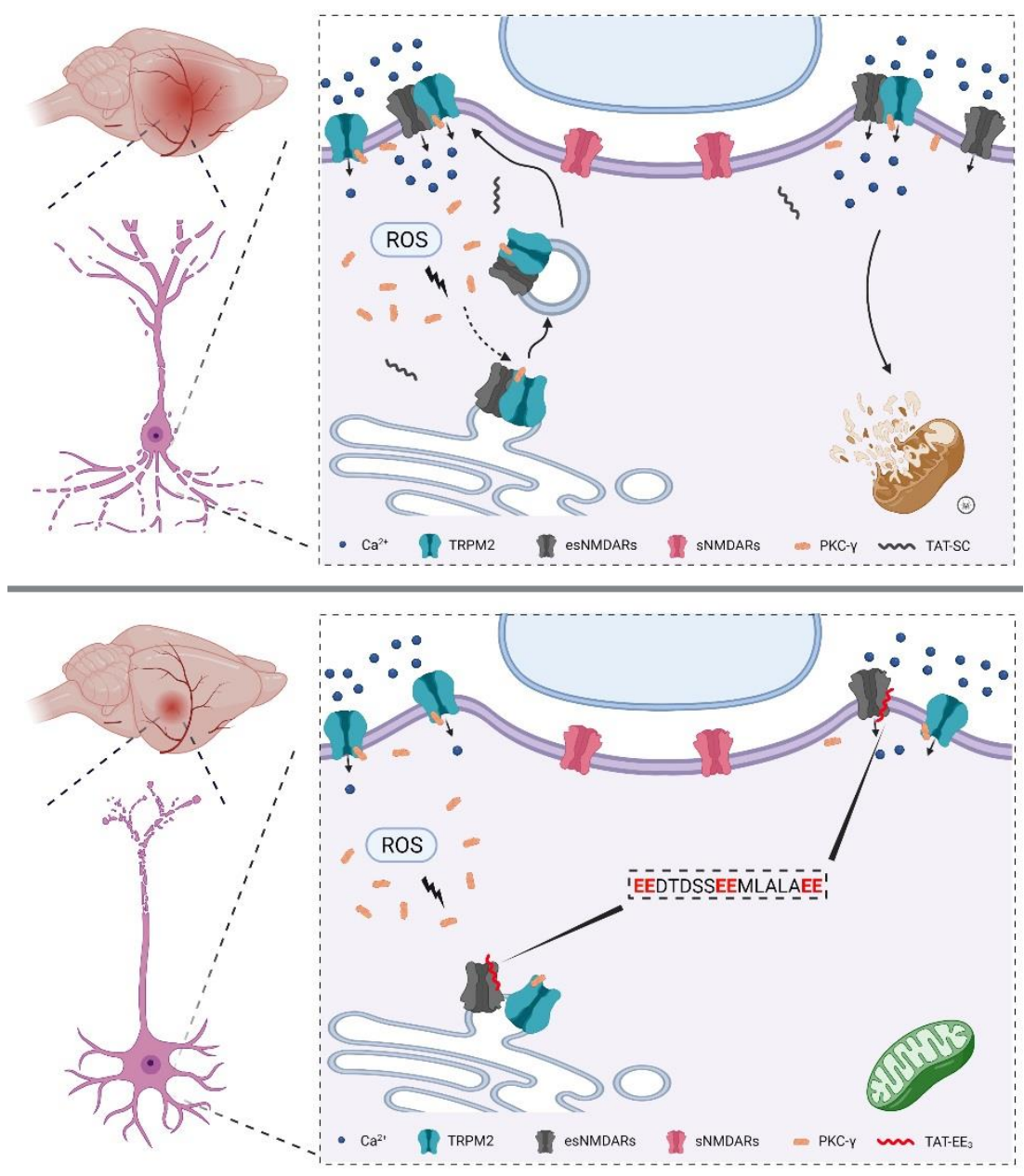

TRPM2 excerbates NMDAR's excitotoxicity by physically and functionally interacting with NMDARs. The disrupting pipette TAT-EE 3 protects neurons against ischemic injury in vitro and in vivo. 


\section{INTRODUCTION}

Neuronal death is a hallmark of ischemic stroke, a devastating neurological disease which remains a leading cause of disability and mortality worldwide (Virani et al., 2020). Numerous factors are involved in neuronal damage during ischemic stroke, among which $\mathrm{Ca}^{2+}$ overload plays a key role in neurotoxicity (Granzotto et al., 2020). $\mathrm{Ca}^{2+}$ overload caused by excitotoxic mechanisms through NMDA receptor (NMDAR) activation and non-excitotoxic $\mathrm{Ca}^{2+}$ entry mechanisms triggers a series of downstream signaling cascades, including reactive oxygen species (ROS) or reactive nitrogen species (RNS) generation, mitochondrial dysfunction, metabolic impairment, and activation of necrosis and apoptosis cascade, and ultimately leads to neuronal death (Choi, 2020). Since it was first discovered fifty years ago (Olney, 1969), excitotoxicity caused by NMDAR-mediated $\mathrm{Ca}^{2+}$ overload has been the center of extensive research for understanding the underlying mechanisms and for developing effective therapeutics for ischemic stroke. However, the development of stroke drugs by antagonizing NMDARs has been characterized by success in animal studies but subsequent failure in clinical trials (Sena et al., 2007).

The lack of clinical success with excitotoxic NMDAR antagonists prompted a shift of the focus of stroke neuroprotection research towards the identification of downstream intracellular signaling pathways triggered by NMDARs (Wu and Tymianski, 2018), and the investigation of subtype-dependent (Ge et al., 2020) as well as localization-dependent excitotoxic effects of NMDARs (Hardingham and Bading, 2010). Over a third of surface NMDARs are located extrasynaptically (Petit-Pedrol and Groc, 2021), which preferentially leads to neurotoxicity and cell death upon activation, whereas activation of synaptic NMDARs promotes a survival mechanism, likely through activation of differential signaling pathways triggered by intracellular $\mathrm{Ca}^{2+}$ (Bading, 2013; Hardingham, 2019). Moreover, the disappointing clinical trial outcome of NMDAR antagonists for stroke treatment also prompted a divergent focus on investigating non-excitotoxic $\mathrm{Ca}^{2+}$-permeable channels as potential therapeutic targets (Tymianski, 2011), including the $\mathrm{Ca}^{2+}$-permeable nonselective transient receptor potential (TRP) channels such as TRPM2 (Belrose and Jackson, 2018). 
TRPM2 was discovered as an oxidative stress-activated $\mathrm{Ca}^{2+}$-permeable non-selective cation channel (Hara et al., 2002; Perraud et al., 2001; Sano et al., 2001), belonging to the TRP superfamily, melastatin subfamily (Clapham, 2003; Montell et al., 2002). A rise of intracellular $\mathrm{Ca}^{2+}\left(\left[\mathrm{Ca}^{2+}\right]_{i}\right)$ and the binding of ADP ribose (ADPR) to the $\mathrm{N}$-terminal binding site (Huang et al., 2019; Huang et al., 2018; Kuhn et al., 2016), and/or C-terminal NUDT9-H domain (Csanady and Torocsik, 2009; Kuhn and Luckhoff, 2004; Perraud et al., 2001; Wang et al., 2018; Yu et al., 2017), triggers conformational changes and opens TRPM2. TRPM2 is susceptible to, and regulated by, stress conditions such as acidic intra- and extracellular pH (Du et al., 2009b; Starkus et al., 2010; Yang et al., 2010), glutathione (GSH) (Belrose et al., 2012), and $\mathrm{Zn}^{2+}$ (Mortadza et al., 2017). TRPM2 is also sensitive to temperature (Kashio et al., 2012) and is involved in body temperature sensation (Kashio and Tominaga, 2017; Song et al., 2016; Tan and McNaughton, 2016; Vilar et al., 2020). The ADPR and $\mathrm{Ca}^{2+-}$-gating features make TRPM2 a common molecular mechanism conferring the susceptibility to cell death induced by ROS and diverse pathological conditions.

TRPM2 is ubiquitously expressed in various cell types and most abundantly in the brain (Fonfria et al., 2006). In response to oxidative stress stimuli, TRPM2-mediated $\mathrm{Ca}^{2+}$ influx leads to cell death of various cell types including neurons (Belrose and Jackson, 2018; Mai et al., 2020; Takahashi et al., 2011). Contribution of TRPM2 to ischemic brain stroke has been demonstrated in ischemia-reperfusion brain damage mouse models (Alim et al., 2013; Gelderblom et al., 2014; Shimizu et al., 2013). However, the reported results have been controversial regarding whether TRPM2 in neurons or TRPM2 in immunocompetent cells plays a key role in causing ischemia-reperfusion brain damage (Alim et al., 2013; Gelderblom et al., 2014; Shimizu et al., 2013), and whether TRPM2 inhibition or knockdown (Jia et al., 2011; Shimizu et al., 2013) only protects against ischemic brain damage in male mice. Nonetheless, although the mechanisms by which TRPM2 results in deleterious effects during ischemic stroke require further investigation, TRPM2 has been implicated as a non-excitotoxic candidate target for ischemic stroke (Belrose and Jackson, 2018; Mai et al., 2020). 
Given the complexity of deleterious effects caused by both excitotoxic $\mathrm{Ca}^{2+}$ and non-excitotoxic $\mathrm{Ca}^{2+}$ signaling pathways during ischemic stroke, blocking just one of these pathways may not be effective in mitigating ischemic injury. We reasoned that a convergent inhibition of the divergent excitotoxic and non-excitotoxic pathways might produce a better therapeutic outcome. Here we unveil a previously unknown mechanism by which TRPM2 mediates deleterious effects leading to neuronal death during ischemic stroke. We found that TRPM2 exacerbates NMDARs' excitotoxicity by physically and functionally interacting with NMDARs triggered by oxidative stress. By discovering the binding domain and designing a disruptive peptide $\mathrm{TAT}-\mathrm{EE}_{3}$, we demonstrated that functional uncoupling of TRPM2 and NMDARs by TAT-EEз 3 protects neurons against ischemic injury in vitro and in vivo. Our results establish that TRPM2 is a molecule converging the excitotoxic and non-excitotoxic pathways. Targeting TRPM2 represents a new therapeutic strategy to eliminate excitotoxicity caused by NMDARs in ischemic stroke.

\section{RESULTS}

\section{TRPM2 deletion in neurons prevents ischemic injury and protects the brain against ischemic stroke}

The oxidative stress-activated TRPM2 is expressed in various types of cells (Fonfria et al., 2006). Inhibition of TRPM2 attenuates ischemic injury, yet the mechanism by which TRPM2 leads to deleterious effects is not fully understood, as both neurons and immune cells were suggested as the primary cause of ischemic injury mediated by TRPM2 (Alim et al., 2013; Gelderblom et al., 2014). To elucidate the underlying mechanisms and determine neuronal damage mediated by TRPM2 during ischemic stroke, we established a neuron-specific Trpm2 deletion model using nestin-cre mice crossed with TRPM2 $2^{\text {fl/1 }}$ mice. Global knockout of TRPM2 (TRPM2-KO) was used as a comparison. Trpm2 deletion was confirmed by detecting TRPM2 protein expression and functional current recording (Figure S1). Using a 120-min middle cerebral artery occlusion (MCAO) followed by reperfusion, we evaluated infarct volume $24 \mathrm{hrs}$ after MCAO by TTC staining. Successful MCAO was confirmed by monitoring blood flow reduction by $85 \%$ (Figure S2). Figure 1 shows that, similar to the protective effects produced by global 
Trpm2 knockout (gM2KO) as previously reported (Alim et al., 2013) (Figure 1A-C), neuron-specific Trpm2 deletion

$\left(\mathrm{Cre}^{+}\right.$, TRPM2 $\left.2^{\mathrm{fl} / \mathrm{f}} ; \mathrm{nM} 2 \mathrm{KO}\right)$ exhibited significantly reduced infarct volume and markedly improved neurological performance in comparison with wild-type (Cre-, TRPM2 $2^{\text {fl/1/1 }}$ WT) littermates (Figure 1D-F). These results establish that TRPM2 in neurons play a key role in mediating neuronal cell death. To further determine the mechanisms of TRPM2-mediated neuronal death during ischemic stroke, we evaluated neuronal cell death by TUNEL staining. At the ischemic penumbra, the numbers of TUNEL-positive neurons were significantly smaller in global TRPM2KO and neuron-specific TRPM2-KO brain slices than those of WT littermates (Figure 1G-L), indicating that attenuation of apoptosis by Trpm2 deletion mediates protective effects against ischemic stroke.

\section{TRPM2 enhances surface expression of NMDARs and exacerbates excitotoxicity}

Various mechanisms are involved in neuronal death, among which $\mathrm{Ca}^{2+}$ overload is a major factor. Using cultured cortical neurons, we applied oxygen-glucose deprivation (OGD) conditions to the neurons to mimic in vivo ischemic injury, and analyzed changes of intracellular $\mathrm{Ca}^{2+}$ and cell death as previously reported (Weilinger et al., 2016). OGD induced a persistent rise of intracellular $\mathrm{Ca}^{2+}$ (Figure $\left.1 \mathrm{M}-\mathrm{O}\right)$ until the lysis of neurons as reflected by a complete loss of Fura-2 fluorescence. The lysed neurons were counted as dead neurons, as neuronal lysis is a hallmark feature of necrosis (Weilinger et al., 2016). Throughout the 90 mins of OGD perfusion, a noticeable number of neurons $(6.8 \%)$ in the WT group died after 30 mins of OGD, and neuron death increased to $35.2 \%$ and $58.5 \%$ at 60 and 90 mins, respectively (Figure $1 \mathrm{M}-\mathrm{P}$ ). In contrast, there was only $1.9 \%, 8.7 \%$, and $16.3 \%$ dead neurons at 30, 60, and 90 mins in the TRPM2-KO group, respectively (Figure 1P), indicating that TRPM2 deletion protects neurons against OGD-induced neuronal death. Similar results were also observed in neurons isolated from neuron-specific Trpm2 deletion Cre+ mice in comparison with Cre- control littermates (Figure S3). Consistent with the higher percentage of neuronal death induced by OGD, the increase in intracellular $\mathrm{Ca}^{2+}$ was also remarkably higher in WT than in TRPM2-KO neurons (Figure 1M-O). It was previously shown that during in vitro 
ischemia of cultured cortical neurons, $80 \%$ of $\mathrm{Ca}^{2+}$ entry is mediated by NMDARs (Goldberg and Choi, 1993; Lipton, 1999). The drastic reduction of $\mathrm{Ca}^{2+}$ entry by Trpm2 deletion (Figure 1M-P) suggests that TRPM2 might have affected NMDAR functions during OGD exposure.

Mitochondrial dysfunction is another hallmark of excitotoxicity and is an early event leading to neuronal death (Keelan et al., 1999; Schinder et al., 1996; Vergun et al., 1999; White and Reynolds, 1996). Dysfunction of mitochondria is characterized by depolarized mitochondria membrane potential caused by opening of the mitochondrial permeability transition pore (mPTP) (Lemasters et al., 2009), which can be monitored by rhodamine 123 (Rh123) fluorescence dequenching assay (Nguyen et al., 1997). OGD-induced mitochondrial depolarization is indicated by increased Rh123 fluorescence in WT neurons, whereas TRPM2 deletion largely prevented mitochondrial depolarization (Figure 1Q-R). Since activation of NMDARs is known to cause mitochondrial depolarization (Abramov and Duchen, 2008; Qiu et al., 2013; Yan et al., 2020), the fact that TRPM2 deletion largely eliminated mitochondrial depolarization during OGD provides another line of evidence suggesting that TRPM2 might have influenced NMDAR function.

Since TRPM2 is sensitive to oxidative stress stimuli, we investigated how TRPM2 is regulated by ischemic stroke in vivo and in vitro. We used sub-optimal $\mathrm{Ca}^{2+}$ and ADPR concentrations (Du et al., 2009a) in the pipette solution for TRPM2 recording in neurons and exposed neurons to OGD. During OGD stimulation, TRPM2 current amplitude was significantly increased in neurons from WT mice (Figure 1S-T), indicating an enhanced channel activity, which might also happen during in vivo ischemic stroke. Moreover, TRPM2 expression level in the WT MCAO brains was 7.6-fold higher than in the WT sham control brains (Figure 1U-V).

As both OGD-induced neural death (Figure 1M-P) and mitochondrial dysfunction (Figure 1Q-R) results suggest that TRPM2 might influence NMDAR functions, we evaluated whether TRPM2 influences NMDAR functions in ischemic stroke in vivo. Using plasma membrane protein extracts from brains of TRPM2-KO (gM2KO) and WT littermate mice subjected to MCAO or sham procedure, we discovered that the surface expression levels of 
NMDARs, including GluN1, GluN2a, and GluN2b, were much higher in the WT MCAO mice in comparison with WT sham control mice. Remarkably, the increase in the surface expression level of NMDARs induced by MCAO mice was almost totally abolished by TRPM2 deletion (Figure 1W-Z). This novel finding prompted us to propose that, as an oxidative stress sensor, TRPM2 influences NMDAR surface expression and function during ischemic stroke, thereby exacerbating NMDAR's excitotoxicity.

\section{TRPM2 interacts with NMDARs}

To understand how TRPM2 may influence NMDAR surface expression in MCAO brains, we tested whether TRPM2 interacts with NMDARs. We first used HEK-293 cells heterologously expressing TRPM2 and the NMDAR subunits GluN1, GluN2a, and GluN2b (GluN1/GluN2a/GluN2b), and performed co-immunoprecipitation (co-IP) experiments. We found that TRPM2 can be pulled down by antibodies specifically against GluN1, GluN2a, and GluN2b, indicating that TRPM2 interacts with the NMDAR protein complex (Figure 2A). In reciprocal co-IP experiments, when TRPM2 was immunoprecipitated by anti-TRPM2, GluN1, GluN2a, and GluN2b were detected in the precipitated complex by western blot (Figure 2B), further indicating that TRPM2 interacts with NMDARs.

Since the subunits GluN1, GluN2a, and GluN2b form a heteromeric channel complex, antibodies against any of these subunits may pull down the entire heterotetrameric complex. To determine which subunits interact with TRPM2, we transfected TRPM2 individually with GluN1, GluN2a, or GluN2b. As shown in Figure 2C, TRPM2 interacted with both GluN2a and GluN2b but not GluN1 when they were separately transfected with TRPM2, indicating that in the GluN1/GluN2a/GluN2b complex, GluN2a and GluN2b subunits interact with TRPM2.

We then sought to determine whether endogenous TRPM2 interacts with the NMDAR complex. We used brain tissues from WT MCAO mice since TRPM2 is highly up-regulated by MCAO (Figure 1U-V). As shown in Figure 2D-E, GluN1, GluN2a, and GluN2b were able to pull down TRPM2, and reciprocally, anti-TRPM2 was able to pull down GluN1, GluN2a, and GluN2b in the WT MCAO brain lysates but not in the TRPM2-KO MCAO brain lysates. 
The interaction between TRPM2 and NMDARs in both exogenous expression systems and brains prompted us to determine the functional significance of the interaction.

\section{Functional coupling between NMDARs and TRPM2}

We tested NMDAR currents in cultured neurons from WT and TRPM2-KO mice. NMDAR currents elicited by 10 $\mu \mathrm{M}$ NMDA at holding potential of $-80 \mathrm{mV}$ were much bigger in WT neurons than that in the TRPM2-KO neurons (Figure 2F). We then tested functional interaction of NMDARs and TRPM2 in the overexpression system. In HEK293T cells overexpressing NMDARs with TRPM2, surface expression levels of GluN1, GluN2a, and GluN2b were higher than that in HEK293T cells overexpressing NMDARs with control EGFP vector plasmids (Figure 2GJ). Consistent with the enhanced surface expression levels, NMDAR currents elicited by NMDA in NMDARs/TRPM2-expressing cells were significantly larger than that in NMDARs/EGFP-expressing cells (Figure 2K-L). The increased surface expression levels and enhanced NMDAR channel functions were also observed in separate transfections when GluN1/GluN2a or GluN1/GluN2b were co-expressed with TRPM2 (Figure S4). These results suggest that TRPM2 and NMDARs physical interactions result in functional coupling reflected by the enhanced functional currents of NMDARs.

\section{Identification of the NMDAR-interacting domain $\mathrm{EE}_{3}$ at the N-terminus of TRPM2}

To understand how TRPM2 interacts with NMDARs, we generated N- and C-terminal fragments of TRPM2 tagged with Flag and GFP, respectively (Flag-TRPM2-NT, GFP-TRPM2-CT). When co-expressed with NMDARs, the TRPM2-N but not the TRPM2-C fragment was detected in the precipitate pulled down by GluN1, GluN2a, and GluN2b antibodies (Figure 3A-B). For NMDARs, the C-termini of GluN2a and GluN2b were pulled down by TRPM2, whereas the C-terminal domain-deleted GluN2a (GluN2a- $\Delta \mathrm{CT}$ ) and GluN2b (GluN2b- $\Delta \mathrm{CT}$ ) were absent in the precipitates pulled down by anti-TRPM2 (Figure 3C). These findings suggest that the C-terminal tail of 
NMARs interact with TRPM2's N-terminal domain.

To determine whether the TRPM2-NT fragment is sufficient to cause functional coupling with NMDARs, we cotransfected NMDARs with full-length TRPM2 (TRPM2-FL), TRPM2-NT, or TRPM2-CT plasmids. NMDAR currents recorded in NMDARs/TRPM2-NT expressing cells were similar to those in NMDARs/TRPM2-FL-expressing cells, whereas the currents in NMDARs/TRPM2-CT group were not different from those recorded in cells expressing NMDARs alone (Figure 3D-E), indicating that TRPM2-NT couples with NMDARs.

To further narrow down the NMDAR-interacting domain at the N-terminus of TRPM2 (Figure 3F), we generated a series of N-terminal truncation constructs by incrementally deleting about 50 residues, and tested which fragments interact with NMDARs (Figure 3G-I). We found that the N-terminal amino acid residues from 631 to 679 are critical for the TRPM2 interaction with NMDARs (Figure 3G-I), as both forward co-IP and reverse co-IP confirmed the interaction for the fragments of $1-727$ and 1-679, whereas the fragments shorter than 631 residues failed to interact with NMDARs. We further deleted the fragment 1-679 and found that the amino acid resides between 665 and 679 are essential for the interaction of TRPM2 and NMDARs (Figure 3J-L). Interestingly, the 15 residues between 665 and 679 contain two "glutamate-glutamate" (EE) repeats separated by five residues and followed by another EE repeat (Figure 3F), so we named the residues between 665 to 681 the "EE3" domain for simplicity. This $\mathrm{EE}_{3}$ domain is present in TRPM2 from different species, but not present in other TRPM channels (Figure S5). When the $\mathrm{EE}_{3}$ domain was deleted from the full-length TRPM2 (TRPM2- $\triangle \mathrm{EE} 3$ ), interaction between TRPM2 and NMDARs was completely disrupted (Figure $3 \mathrm{M}$ ). Intriguingly, when the middle "EE" of the $\mathrm{EE}_{3}$ domain was replaced by "QQ" (glutamine), the TRPM2-EQE mutant failed to interact with NMDARs, whereas mutations of the first and third EE repeats (TRPM2-QEE, TRPM2-EEQ) did not influence the TRPM2-NMDARs interaction (Figure 30). These results indicate that the EE residues in the middle of the EE3 domain are critical for the TRPM2NMDAR interaction.

To investigate the functional consequence of disrupting the physical interaction between TRPM2 and NMDARs, 
wild-type TRPM2 or TRPM2 $\mathrm{EE}_{3}$ mutants were co-expressed with NMDARs in HEK293 cells for current recording. Consistent with the disrupted interaction, TRPM2- $\triangle E E 3$ and TRPM2-EQE mutants failed to enhance NMDAR currents, whereas TRPM2-QEE and TRPM2-EEQ mutants increased NMDAR currents, similar to the potentiation of NMDAR currents induced by WT-TRPM2 when co-expressed with NMDARs in HEK-293T cells (Figure 3N \& 3P). It is noteworthy that the TRPM2 mutations did not affect TRPM2 channel function (Figure S5). These results indicate that the $\mathrm{EE}_{3}$ domain is essential for physical and functional coupling between TRPM2 and NMDARs.

\section{Mechanisms of TRPM2 and NMDARs functional coupling}

The above results indicate that TRPM2 interacts with NMDARs through the TRPM2-EE 3 domain thereby enhancing NMDAR function by increasing its surface localization (Figure $2 \& 3$ ). Next, we asked how the interaction of TRPM2 with NMDARs enhances surface expression of NMDARs. Since it has been previously shown that PKC regulates NMDAR trafficking to the cell surface (Lan et al., 2001; Zheng et al., 1999), we reasoned that PKC could be part of the NMDAR-TRPM2 complex. Thus, we investigated whether PKC interacts with TRPM2. Using WT and global TRPM2-KO (gM2KO) mouse brains after MCAO, we found that neuron-specific PKC $\gamma$ can be readily pulled down by anti-TRPM2 in WT but not in TRPM2-KO (gM2KO) brains (Figure 4A). In HEK293 cells over-expressing PKC $\gamma$ with full-length TRPM2 (TRPM2-FL), TRPM2-CT, or TRPM2-NT, we found that TRPM2-FL and TRPM2-NT, but not TRPM2-CT, pulled down PKC $\gamma$, indicating that PKC $\gamma$ interacts with TRPM2-NT (Figure 4B). Moreover, when we used brain tissues from both sham and MCAO mice, the amount of PKC $\gamma$ pulled down by anti-TRPM2 antibody in MCAO mice was about 1.5 -fold of that from sham mice (Figure 4CD), suggesting that oxidative stress during MCAO increased TRPM2 and PKC $\gamma$ interaction. Indeed, cultured neurons treated with $\mathrm{H}_{2} \mathrm{O}_{2}$ exhibited significantly increased TRPM2 and $\mathrm{PKC} \gamma$ interaction (Figure 4E-F).

In order to investigate whether PKC $\gamma$ influences surface expression levels of NMDARs, we co-expressed NMDARs and TRPM2 with wild-type PKC $\gamma$ or dominant-negative PKC $\gamma(\mathrm{PKC} \gamma$-DN). As shown in Figure 4G-J, 
over-expression of PKC $\gamma$ further increased surface expression of NMDARs, whereas dominate-negative PKC $\gamma$ abolished the increase of surface NMDAs by TRPM2, suggesting that a functional PKC $\gamma$ is required for increased NMDARs trafficking induced by TRPM2.

We also used pharmacological tools to probe the function of PKC in the TRPM2-mediated enhancement of surface NMDAR levels. HEK293T cells transfected with NMDARs and TRPM2 exhibited much higher level of surface NMDARs after treatment with PKC activator PMA (Figure 4K-L), whereas PKC inhibitor staurosporine abolished the enhanced surface expression level of NMDARs (Figure 4M-N). The effects of PKC on NMDARs were further confirmed by the effects of PKC inhibitor staurosporine on NMDAR currents recorded in the neurons from WT mice. Staurosporine normalized NMDAR current amplitude in the WT neuron to the similar amplitude level of NMDAR current in TRPM2-KO neurons (Figure 4O-P).

As trafficking of NMDARs to plasma membrane involves exocysts (Sans et al., 2003), we tested the effects of blocking exocysts on NMDARs surface expression. Endosidin 2, an exocyst inhibitor, prevented the increased surface expression level of NMDARs (Figure S6) induced by co-expression with TRPM2, and abolished the increased current amplitude of NMDARs by TRPM2 in WT neurons (Figure 4Q-R).

Taken together, the above results indicate that PKC $\gamma$ interacts with TRPM2, which can be promoted by an oxidative stress condition and by MCAO in vivo, and that interaction of PKC $\gamma$ with TRPM2 enhances NMDARs surface trafficking likely via exocysts.

\section{Disrupting peptide eliminates physical interaction and functional coupling of TRPM2 and NMDARs}

As the TRPM2 N-terminal $\mathrm{EE}_{3}$ domain is critical for TRPM2 and NMDARs interaction, we designed membranepermeable peptides, TAT-EE 3 and scrambled control peptide TAT-SC, to investigate whether disruption of physical interaction eliminates the functional coupling between TRPM2 and NMDARs. To determine whether TAT$\mathrm{EE}_{3}$ is able to disrupt the interaction of TRPM2 and NMDARs, we conducted co-IP experiments using NMDARs 
co-expressed with WT-TRPM2 and TRPM2 EE 3 mutants including TRPM2-QEE, TRPM2-EQE, TRPM2-EEQ, and the deletion mutant TRPM2- $\triangle \mathrm{EE}_{3}$. Similar to the TRPM2- $\Delta(\mathrm{EE})_{3}$ and TRPM2-EQE mutants, TAT-EE 3 treatment completely disrupted the interaction of TRPM2 with GluN2a (Figure 5A) and GluN2b (Figure 5B), whereas TAC-SC treatment and mutants TRPM2-QEE and TRPM2-EEQ did not influence TRPM2 and GluN2a and GluN2b interactions. We further determined the surface expression of NMDARs after TRPM2- and NMDARoverexpressing cells were treated with TAT-EE 3 or TAT-SC. TAT-EE 3 eliminated the enhancement of NMDAR surface expression by TRPM2 (Figure 5D, F), whereas TAT-SC exhibited no influence (Figure 5C, E). Consistent with the disrupted interaction between TRPM2 and NMDARs and the eliminated increase of NMDAR surface expression, the NMDAR current amplitude in NMDARs and TRPM2-overexpressing cells treated with TAT-EE 3 was significantly smaller than that in cells treated with TAT-SC (Figure 5G-H).

As PKC activation can both increase NMDAR trafficking to the cell surface and regulate channel activity (Lan et al., 2001), we sought to determine the PMA effects on NMDAR currents in neurons treated with TAT-EEз or TATSC. Cultured neurons from WT and TRPM2-KO mice were treated with TAT-EE 3 or TAT-SC overnight. NMDAR currents were elicited before and after 20 s perfusion with PMA. As shown in Figure $5 \mathrm{I}$ (top left), NMDA-induced current was increased from 1542.1 $\pm 117.1 \mathrm{pA}$ to $3642.1 \pm 180.8 \mathrm{pA}$ (Figure 5J) by PMA perfusion for $20 \mathrm{~s}$ (Figure 5J-K), about a 1.5-fold increase, in neurons from WT mice. However, in the neurons incubated with TAT-EE $E_{3}$, the NMDAR current amplitude was much smaller before and after PMA perfusion (Figure 5I, top right and Figure 5J), and the increase of current amplitude induced by PMA was also significantly smaller than that in neurons incubated with TAT-SC (Figure 5K). The smaller NMDAR currents before PMA and smaller increase of NMDAR currents after PMA in TAT-EE 3 -incubated neurons suggest that disruption of TRPM2 and NMDARs interaction may have affected surface trafficking of NMDAR-induced PKC activation. In the neurons from global TRPM2-KO (gM2KO) mice, the NMDAR current amplitude (Figure 5I, bottom) and PMA-induced increase in NMDAR currents were much smaller than that of WT neurons. Moreover, there was no difference in PMA-induced changes of 
NMDAR currents between TAT-SC and TAT-EE 3 incubated neurons from TRPM2-KO mice (Figure 5J-K). These results indicate that similar to TRPM2-KO, disruption of the interaction between TRPM2 and NMDARs in the WT neurons by TAT-EE 3 reduced NMDAR currents, and attenuated the PMA-induced increase in NMDAR currents. As PMA causes an increase of NMDAR channel activities and surface trafficking, it is conceivable that in TRPM2KO and TAT-EE 3 -treated neurons, PMA failed to cause surface trafficking of NMDARs. This notion is further supported by the fact that inhibition of exocysts abolished the increase of surface expression of NMDARs coexpressed with TRPM2 in HEK293T cells (Figure S6), and the enhancement of NMDAR currents by TRPM2 in WT neurons (Figure 4Q-R).

\section{Disruption of TRPM2 and NMDAR interaction protects neurons against ischemic injury in vitro}

The functional uncoupling of TRPM2 from NMDARs by TAT-EE 3 prompted us to investigate its significance during ischemic injury. Using cultured neurons, the disrupting peptide TAT-EE 3 efficiently reduced NMDAR currents in neurons from WT mice but not from TRPM2-KO mice, whereas TAT-SC exhibited no influence on NMDAR currents from WT and TRPM2-KO neurons (Figure 6A-B), indicating the specificity of TAT-EE 3 . To mimic in vivo ischemia conditions, we used OGD to treat cultured neurons. When neurons were exposed to OGD, the changes of intracellular $\mathrm{Ca}^{2+}$ were significantly reduced in WT but not in TRPM2-KO neurons by pretreatment with TAT$\mathrm{EE}_{3}$ (Figure 6C-D). The OGD-mediated neuronal death was $10.6 \%, 47.6 \%$, and $70.5 \%$ at 30,60 , and 90 min,

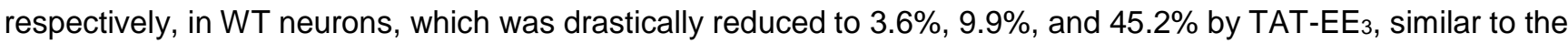
percentage death rate in the global TRPM2-KO (gM2KO) neurons (Figure 6E).

We next evaluated whether TAT-EE3 influences OGD-mediated mitochondrial dysfunction using the Rh123 fluorescence de-quenching assay (Nguyen et al., 1997). As shown in Figure 6F-G, OGD caused mitochondria depolarization as reflected by the increase of Rh123 fluorescence in WT neurons treated with TAT-SC. In contrast, mitochondrial membrane depolarization induced by OGD was markedly inhibited in WT neurons treated with TAT- 
$\mathrm{EE}_{3}$, similar to the inhibited depolarization in neurons from global TRPM2-KO (gM2KO) or neuron-specific TRPM2 deletion (nM2KO) mice (Figure 6F-G). The mitochondria membrane depolarization was mediated by $\mathrm{Ca}^{2+}$ entry because when neurons were perfused with $\mathrm{Ca}^{2+}$ free $\mathrm{OGD}$, membrane depolarization was inhibited (Figure 6F: micrographs at $2^{\text {nd }}$ row from bottom, and Figure $6 \mathrm{G}$ ). These results indicate that the TAT-EE 3 disrupting peptide abolishes the exacerbation of NMDAR excitotoxicity that results from functional coupling of TRPM2 and NMDARs, and protects neurons from ischemic injury in vitro.

\section{Disruption of TRPM2 and NMDAR interaction protects mice against ischemic stroke}

To determine if TAT-EE 3 inhibits ischemic injury in vivo, we administrated TAT-EE 3 or TAT-SC $(100 \mathrm{nmol} / \mathrm{kg}) 15$ mins before MCAO or sham surgery as previously reported (Weilinger et al., 2016). Infarct volume and neurological deficit scores were evaluated 24 hrs after MCAO (see description for Figure 1). As shown in Figure 6H-J, TAT-TTEз-treated mice exhibited significantly reduced infarct volume and improved neurological behavior scores in comparison with TAT-SC-treated mice. Similar to those in the global (gM2KO) (Figure 1W) and neuronal (nM2KO) TRPM2-KO mice (Figure 6K), the enhanced surface expression of NMDARs was abolished by TAT-EE 3 (Figure 6K-L) in MCAO mice, indicating that disruption of NMDARs and TRPM2 coupling is an effective approach to protect the brain against ischemia injury.

As synaptic NMDARs promote pro-survival signals whereas extrasynaptic NMDARs promote pro-death signals (Hardingham and Bading, 2010; Hardingham et al., 2002), we sought to determine whether the enhanced NMDAR surface expression by MCAO in WT mice is pro-survival or pro-death. Cultured neurons from global TRPM2-KO, neuronal TRPM2-KO (Cre+), or Cre- WT littermates (WT) were treated for $1 \mathrm{~h}$ with NMDA to activate both synaptic and extra-synaptic NMDARs, or 4-AP $(2.5 \mathrm{mM})$ plus $50 \mu \mathrm{M}$ bicuculline (Bic) to activate only synaptic NMDARs (Hardingham et al., 2002; Nicolai et al., 2010). WT neurons were pre-incubated with TAT-SC or TAT-EE 3 overnight. After induction of NMDAR activation by NMDA or 4-AP/Bic, neurons were harvested for quantitative analysis of 
CREB and ERK1/2 activation. In neurons pre-incubated with TAT-SC, treatment with 4-AP/Bic significantly increased neuronal survival signal, as indicated by the phosphorylated ERK1/2 (pERK1/2) and phosphorylated CREK (pCREB) in comparison with control group, whereas NMDA treatment drastically reduced the pro-survival signal pERK1/2 and pCREB in WT neurons treated with TAT-SC group (Figure 6M-O), similar to previous findings in neurons without any pre-incubation (Hardingham et al., 2002; Nicolai et al., 2010). In stark contrast, both 4AP/Bic and NMDA treatments increased pro-survival signal ( $p E R K 1 / 2$ and $p C R E B$ ) levels in WT neurons preincubated with $\mathrm{TAT}_{-E_{3}}$, as well as in neurons from mice with global or neuronal-specific TRPM2 deletion, indicating that disruption of the TRPM2-NMDAR interaction by TAT-EE 3 or by deletion of TRPM2 largely eliminated extrasynaptic activation of NMDARs by NMDA.

To determine whether TRPM2 exacerbates NMDAR extrasynaptic excitotoxicity in WT MCAO mice, we further analyzed CREB and ERK1/2 activities in brain tissues from MCAO mice treated with TAT-SC or TAT-EE3. In TAT-SC-treated mice, pERK1/2 and pCREB levels were significantly lower in MCAO brains than sham control brains, whereas MCAO induced no reduction in pERK1/2 and pCREB levels in TAT-EE $E_{3}$-treated brains (Figure 6P-R). Similarly, neuronal TRPM2-KO (Figure 6P-R) and global TRPM2-KO (Figure S7A-C) prevented reduction of $\mathrm{pERK} 1 / 2$ and $\mathrm{pCREB}$ levels after MCAO, indicating that in vivo disruption of TRPM2-NMDAR interaction, as well as deletion of TRPM2, protects brain against ischemia injury likely by inhibiting the extrasynaptic NMDARinduced large reduction in pro-survival signals.

\section{DISCUSSION}

In this study, we report a previously unknown mechanism underlying ischemic brain stroke. We discovered that the up-regulation of TRPM2 during ischemic stroke is physically and functionally coupled to NMDARs, which results in enhanced extrasynaptic NMDAR activity and thereby leads to increased excitotoxicity. We revealed that the mechanism by which TRPM2 increases extrasynaptic NMDAR excitotoxicity is through interaction between 
TRPM2 and PKC $\gamma$, which leads to increased NMDAR protein trafficking and surface expression. Moreover, we identified the specific binding domain in the N-terminus of TRPM2 for coupling of TRPM2 with NMDARs, and designed a membrane-permeable disrupting peptide to uncouple TRPM2 from NMDARs. By disrupting the TRPM2-NMDAR interaction, the peptide TAT-EE 3 not only inhibited OGD-induced excitotoxicity in vitro, but also efficiently protected mice against ischemic stroke injury in vivo. Our results indicate that by exacerbating the excitotoxicity of NMDARs, TRPM2 converges both excitotoxic and non-excitotoxic $\mathrm{Ca}^{2+}$ signaling pathways in mediating neuronal death in ischemic stroke. This new mechanism may serve as a foundation for designing and developing effective strategies for future ischemic stroke therapies.

\section{TRPM2 in neurons plays a key role in ischemic injury by exacerbating NMDARs excitotoxicity}

The $\mathrm{Ca}^{2+}$-permeable TRPM2 was discovered as an oxidative stress-activated non-selective cation channel (Hara et al., 2002; Perraud et al., 2001; Sano et al., 2001). Although TRPM2 has been implicated in ischemic stroke (Belrose and Jackson, 2018), the mechanisms by which TRPM2 mediates ischemic injury have been controversial. Some studies suggest that neuronal TRPM2 contributes to ischemic injury (Alim et al., 2013; Jia et al., 2011), whereas others report that TRPM2 expressed in immune cells contributes (Gelderblom et al., 2014). As all the previous studies used global TRPM2-KO (gM2KO), we established neuron-specific TRPM2 knockout (nM2KO) and found that neuronal TRPM2 plays a key role in mediating ischemic brain injury. Our results not only establish that TRPM2 expressed in neurons is critical for mediating ischemic injury, but also reveal a previously unknown mechanism that TRPM2 exacerbates NMDAR excitotoxicity in mediating neuronal death during ischemic injury.

\section{A specific domain mediates physical and functional coupling of TRPM2 with NMDARs and exacerbation}

\section{of NMDAR's excitotoxicity}

Cerebral ischemic injury is characterized by excitotoxicity caused by overactivation of NMDARs which leads to mitochondria dysfunction (Schinder et al., 1996), and $\mathrm{Ca}^{2+}$ overload results from both excitotoxic and non- 
excitotoxic $\mathrm{Ca}^{2+}$ signaling pathways (Choi, 2020). The excitotoxic glutamate-dependent NMDAR channels have been targets for stroke intervention for over 50 years (Choi, 2020). TRPM2 is one of the glutamate-independent, non-excitotoxic $\mathrm{Ca}^{2+}$ channels which has been implicated as a potential target for ischemic stroke in recent years (Tymianski, 2011). The intriguing result in this study is that, as a non-excitotoxic $\mathrm{Ca}^{2+-}$ permeable channel, TRPM2 exacerbates NMDAR's excitotoxicity, implying that intervention of TRPM2 may attenuate both non-excitotoxicity as well as excitotoxicity during ischemic stroke.

How could TRPM2 converge the non-excitotoxic $\mathrm{Ca}^{2+}$ signaling and NMDAR-dependent excitotoxic $\mathrm{Ca}^{2+}$ signaling pathways to mediate neuronal death during MCAO? We revealed that TRPM2 physically interacts with GluN2a and GluN2b in both heterologous-expressing HEK293T cells and in mouse brains. The physical interaction leads to enhanced surface expression and increased current amplitude of NMDARs. More importantly, we uncovered that the C-termini of GluN2a and GluN2b bind to the $\mathrm{EE}_{3}$ domain at the N-terminus of TRPM2, which is a stretch of 16 residues containing three "EE" repeats separated by five residues. When the physical interaction is disrupted by mutations or truncation of the $\mathrm{EE}_{3}$ domain, or by the disrupting peptide TAT-EE $\mathrm{E}_{3}$, interaction of TRPM2 and NMDARs in HEK-293T cells or in neurons is abolished, leading to elimination of both the enhanced surface expression of NMDARs and its increased channel activity (Figure 2-3).

\section{TRPM2 controls NMDAR surface expression by interacting with $P K C \gamma$ during ischemic stroke}

NMDARs have been shown to be regulated by PKC through modulating intrinsic channel properties and NMDAR trafficking (Carroll and Zukin, 2002; Lan et al., 2001; Xiong et al., 1998). Interestingly, we found that the neuronspecific isoform of PKC, PKC $\gamma$, interacts with TRPM2 through the N-terminal domain of TRPM2. More importantly, the interaction of TRPM2 and PKC $\gamma$ was significantly increased in MCAO brains in vivo, and by $\mathrm{H}_{2} \mathrm{O}_{2}$ in the heterologous expressing HEK293T cells in vitro, indicating that oxidative stress and ischemic injury conditions promote TRPM2 and PKC $\gamma$ association (Figure 4). Consistent with our results, oxidative stress-induced interaction 
of PKCa with a non-functional shorter isoform of TRPM2 (the alternative slice variant, TRPM2-S) was reported in a previous study, albeit full-length TRPM2 was found not to interact with PKCa (Hecquet et al., 2014). As PKC modulates NMDAR trafficking, it is conceivable that increased interaction of PKC $\gamma$ and TRPM2 under oxidative stress conditions underlies the mechanism of elevated surface expression of NMDARs in MCAO mouse brains. PKC-mediated NMDAR trafficking to the cell surface has been proposed with different mechanisms. Some studies demonstrated that PKC mediates NMDAR surface trafficking through phosphorylation of serine residues (Ser896 and Ser897) in close proximity to the ER-retention motifs of GluN1 (Horak and Wenthold, 2009; Scott et al., 2001; Standley et al., 2000), whereas others showed that PKC-induced increase of NMDAR activity is not mediated by phosphorylation of NMDARs (Zheng et al., 1999). The latter is supported by another study showing that PKCinduced NMDAR trafficking is mediated by triggering auto-phosphorylation of CaMKII, which is associated with NMDARs (Yan et al., 2011). Nonetheless, it seems that phosphorylation function is important for PKC to mediate NMDAR trafficking regardless of whether it directly phosphorylates NMDARs or indirectly phosphorylates their partners. Indeed, whereas PKC $\gamma$ co-expressed with TRPM2 and NMDARs caused enhancement of NMDARs surface expression, the dominate-negative PKC $\gamma$ failed to do so (Figure 4). Moreover, PKC activator PMA induced higher NMDARs surface expression, whereas PKC inhibitor staurosporine blocked PKC-induced effects (Figure 4). The effects of PKC $\gamma$ in the absence of PMA could be attributed to the basal activity under cell culture conditions when cells are surrounded with various growth factors. As both PKC $\gamma$ and GluN2a/GluN2b interact with the Nterminus of TRPM2 (Figures 3-4), it is conceivable that under oxidative stress conditions, the increased binding of $\mathrm{PKC} \gamma$ to TRPM2 (Figure 5E-F) may bring PKC $\gamma$ in closer proximity to NMDARs or their interacting protein partners, thereby increasing PKC $\gamma$-induced phosphorylation and subsequent surface trafficking of NMDARs.

Another line of evidence supporting the notion of PKC $\gamma$-mediated TRPM2-NMDAR complex trafficking to the cell surface is the inhibitory effects produced by endosidine2, an inhibitor of one component of the exocyst complex (Sans et al., 2003), EXO70 (Zhang et al., 2016). NMDARs interact with the exocyst (Sans et al., 2003) for PKC- 
induced surface delivery via a secretory pathway (Hirschberg et al., 1998). We found that endosidine2 abolished TRPM2-induced enhancement of NMDAR surface expression, and inhibited NMDAR currents in neurons from WT mice (Figure 4Q-R, Figure S6), suggesting that TRPM2-induced NMDAR surface trafficking involves a PKC activation-induced secretory pathway. Furthermore, disruption of the TRPM2-NMDAR interaction largely eliminated increases in surface expression of NMDARs as well as functional NMDAR currents elicited by PMA (Figure 5), strongly supporting that the PKC $\gamma / T R P M 2 / N M D A R$ complex is critical for NMDAR surface trafficking. Although further studies are needed to fully understand the exact mechanism by which $\mathrm{PKC} \gamma$ mediates trafficking of the TRPM2/NMDAR complex, we propose the following working model based on our results. Interactions of TRPM2 with NMDARs and PKC $\gamma$ create a microenvironment where releasing of NMDARs from ER and trafficking of NMDARs to the cell surface are significantly increased under oxidative stress conditions, conferring enhanced excitotoxicity during cerebral ischemic injury.

\section{Functional coupling of TRPM2 to NMDARs exacerbates extrasynaptic excitotoxicity during ischemic}

\section{injury}

Our data show that functional coupling between TRPM2 and NMDARs appears to only exacerbate extrasynaptic NMDAR excitotoxicity. Using the disrupting peptide $T A T-E_{3}$, we found that $T A T-E_{3}$ not only effectively eliminated the increase of NMDAR surface expression and functional increase of NMDAR currents induced by PKC activation (Figures 5-6), but also sufficiently eliminated OGD-induced excitotoxicity in vitro (Figure 6), and significantly attenuated MCAO-induced neuron death in vivo (Figure 6). Intriguingly, TAT-EE 3 prevented the decreases in pERK1/2 and PCREB levels in OGD-treated neurons and MCAO brains, a hallmark of pro-survival signaling pathway which can be shut off by extrasynaptic NMDAR activation during ischemic injury (Hardingham and Bading, 2010; Hardingham et al., 2002). Similar to what we demonstrated, pCREB level can be reduced by more than 50\% 24 hrs after MCAO (Zhang et al., 2020). The ability of TAT-EE 3 and TRPM2 knockout to prevent 
the decreases in PCREB and pERK1/2 levels in MCAO mice strongly indicates that disruption of TRPM2 and NMDAR coupling largely eliminates extrasynaptic excitotoxicity during ischemic brain injury.

It is not surprising that the TRPM2-NMDAR coupling only exacerbates extrasynaptic NMDAR functions (Figure 6), as TRPM2 is absent from the synapse based on the synaptome databases (Bayes et al., 2012). Other studies have also demonstrated a predominantly extrasynaptic distribution of TRPM2 in cultured hippocampal neurons (Olah et al., 2009). Moreover, a previous study demonstrated that NMDAR expression in hippocampal slices from mice not subjected to MCAO was not influenced by TRPM2-KO (Xie et al., 2011), which is consistent with our results showing that NMDAR surface expression level in sham mice is not different between WT and TRPM2-KO (Figure 1W). Thus, it is unlikely that TRPM2-KO can cause abnormal functions of NMDARs under normal physiological conditions. Indeed, TRPM2-KO mice were behaviorally indistinguishable from WT littermates (Yamamoto et al., 2008), which is consistent with our observation that global TRPM2-KO as well as neuronspecific TRPM2-KO mice behave the same as their WT littermates. Furthermore, TRPM2 only enhanced NMDAR function under ischemic injury conditions, which promotes PKC $\gamma$ to interact with TRPM2 and subsequently NMDAR trafficking to the cell surface. Therefore, disrupting the interaction between TRPM2 and NMDARs to specifically target extrasynaptic NMDAs and to mitigate ischemic stroke will unlikely generate side effects caused by directly antagonizing NMDARs.

\section{Disrupting peptide TAT-EE 3 protects neurons against ischemic injury in vitro and in vivo}

The most exciting result in this study is the effectiveness of the disruption of NMDAR-TRPM2 coupling in protecting mice against ischemic brain stroke. Cell-permeable peptides such as TAT-fused peptides have been well characterized and are considered powerful tools for both medical applications and fundamental basic research (Xie et al., 2020). By disrupting the TRPM2-NMDAR interaction, TAT-EE $E_{3}$ effectively eliminated the enhanced extrasynaptic toxicity of NMDARs induced by TRPM2. In neurons treated with $10 \mu \mathrm{M}$ TAT-EE 3 and in 


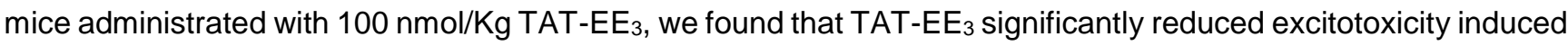
by OGD, prevented the reduction of pCREB and pERK1/2 level in OGD-treated neurons and MCAO mice, and reduced infarct volume as well as neurological deficits in MCAO mice (Figure 6 and Figure S7). These results indicate that disrupting TRPM2 and NMDAR coupling is a promising therapeutic strategy for ischemic stroke.

Although NMDARs interact with various proteins (Petit-Pedrol and Groc, 2021), including recently reported TRPM4 (Yan et al., 2020), there are several unique aspects about the TRPM2-NMDAR interaction. First, TRPM2 is an oxidative stress-activated $\mathrm{Ca}^{2+}$-permeable channel; second, as an ion channel interacting with NMDARs, TRPM2 alters NMDAR surface expression by enhancing NMDAR surface trafficking; third, TRPM2 orchestrates the PLC $\gamma$ to NMDAR interacting complex to enhance NMDAR surface trafficking; fourth, TRPM2 exacerbates extrasynaptic NMDAR function to increase neuronal death. Since TRPM2 is a non-excitotoxic $\mathrm{Ca}^{2+}$-permeable channel, the TRPM2-NMDAR interaction-induced exacerbation of NMDAR excitotoxicity makes TRPM2 a unique molecule that converges non-excitotoxic and excitotoxic $\mathrm{Ca}^{2+}$ signaling pathways, which may serve as an effective target for ischemic stroke.

\section{Translational implications}

Our data in this study provide a new strategy of targeting excitotoxicity without directly antagonizing NMDARs. Excitotoxicity by glutamate acting on NMDARs has long been established as the dominant conceptual model underlying neuronal cell death associated with ischemic stroke. However, the outcomes from clinical trials of directly antagonizing NMDARs by NMDAR antagonists have been disappointing (Choi, 2020). The adverse effect of inhibiting NMDAR physiological functions is one of many factors contributing to failed NMDAR antagonists in translational applications. In this study, we show that disrupting the physical and functional coupling of NMDARs and TRPM2 can largely eliminate the extrasynaptic toxicity of NMDARs during ischemic injury while preserving the pro-survival synaptic NMDAR function. Thus, targeting TRPM2 may represent an effective strategy for future 
design and development of treatments for ischemic stroke.

In summary, we found that TRPM2 in neurons plays a key role in mediating the deleterious effects of TRPM2 in ischemic brain stroke. We uncovered that physical and functional coupling of TRPM2 with NMDARs leads to enhanced extrasynaptic NMDAR toxicity under oxidative stress conditions. We revealed that interaction of TRPM2 with PKC $\gamma$ underlies the mechanism by which TRPM2 mediates enhancement of NMDAR surface expression and functional increase of NMDAR currents. We identified a specific NMDAR-binding domain of TRPM2, and designed a membrane-permeable disrupting peptide TAT-EE 3 . We demonstrated that uncoupling TRPM2 from NMDARs leads to protective effects in vitro and in vivo. As TRPM2 is a non-excitotoxic $\mathrm{Ca}^{2+}$-permeable channel, our results provide a new conceptual strategy that targeting TRPM2 can eliminate ischemic neuronal toxicity mediated by both non-excitotoxic and excitotoxic $\mathrm{Ca}^{2+}$ signaling pathways and protect animals against ischemic stroke.

\section{STAR * METHODS}

Detailed methods are provided in the online version of this paper and include the following:

- KEY RESOURCE TABLE

- $\quad$ CONTACT FOR REAGENT AND RESOURCE SHARING

- EXPERIMENTAL MODEL AND SUBJECT DETAILS

- Animal models

- Cortical neuronal cultures

- Cultured cell lines

- METHOD DETAILS

- Knockout of TRPM2 in mice

- Middle cerebral artery occlusion (MCAO, filament method)

- Neurological deficit score evaluation

- Infarct volume assessment by Triphenyl Tetrazolium chloride (TTC) staining

- Subcloning of TRPM2 and NMDARs

- Neuron isolation, culture and treatment 
- In vitro oxygen-glucose deprivation model

- Cell culture and transfection

- Real-time monitoring of mitochondrial function

- Ratio calcium imaging experiments

- Surface protein isolation

- Protein extraction and immunoblotting

- Co-immunoprecipitation

$\circ$ Electrophysiology

- Immunofluorescence staining

- QUANTIFICATION AND STATISTICAL ANALYSIS

- DATA AND SOFTWARE AVAILBILITY

\section{ACKNOWLEDGMENTS}

We would like to thank Drs Rajkumar Verma (UCONN Health) and Louise McCullough (UT Health) for constructive discussions about this project. We thank Dr. Rindy Jaffe for helpful comments to the manuscript. We thank Dr. Andrew M. Scharenberg (University of Washington) for kindly providing TRPM2 plasmid.

This work was partially supported by the National Institute of Health (R01-HL143750) and American Heart Association (19TPA34890022) to LY.

\section{AUTHOR CONTRIBUTIONS}

L.Y. conceived and designed the research. P.Z. designed and performed most of the in vitro experiments. Z.Y. and J.F. performed most of the in vivo experiments. Z.Y., B.S., Y.H., Z.S., A.S.Y., and J.X. conducted some of the in vitro experiments. G.W. conducted MCAO surgeries and supervised others doing in vivo MCAO surgeries. B.M. and Y. M. generated knockout mice and provided input to discussion of the research. P.Z. and J.F. helped in preparation of the manuscript. L.Y. wrote the manuscript with help from P. Z. All authors commented on the manuscript.

\section{DECLARATION OF INTERESTS}

The authors declare no competing financial interests related to this work. 


\section{FIGURE LEGENDS}

Figure 1 | Neuron-specific Trpm2 knockout protects the brain against ischemic damage via reducing

\section{excitotoxicity during ischemia stroke}

(A-C), Global Trpm2 deletion (gM2KO) reduces infarct volume and improves neurological deficit (ND) score in mice subjected to MCAO. In (A), representative images of TTC staining of slices at $1 \mathrm{~mm}$ thickness from brains of wild-type (WT) and gM2KO mice 24 hrs after sham or MCAO surgery. In (B), mean infarct volume after MCAO from WT $(n=12)$ and gM2KO brains $(n=15)$. In $(C)$, average ND score 24 hrs after MCAO from 12 WT and 15 gM2KO mice ${ }^{* * *}: p<0.001 ;$ ANOVA, Bonferroni's test; mean \pm SEM).

(D-F), Neuron-specific Trpm2 deletion by nestin Cre (Cre ${ }^{+}$nM2KO for simplicity) produces similar protective effects to that of gM2KO for ischemic stroke as shown in (A-C). (D), Representative images of TTC staining of $\mathrm{Cre}^{+}$Trpm2 knockout mice (nM2KO) and Cre- control littermates (WT: for simplicity) 24 hrs after sham or MCAO surgery. (E), Average infarct volume after MCAO from $15 \mathrm{WT}$ and $15 \mathrm{nM} 2 \mathrm{KO}$ mouse brains $\left({ }^{* * *}, p<0.001\right.$; ANOVA, Bonferroni's t-test, mean \pm SEM). $(F)$, Mean ND score of the 15 nM2KO and WT mice $24 \mathrm{hrs}$ after MCAO.

(G-L), Neuronal death evaluated by TUNEL staining of brain sections from gM2KO versus WT mice (G-I) and nM2KO versus Cre- control WT littermates. $(G, J)$, Representative merged images of TUNEL staining of brain sections of WT and M2KO (G), and brain sections of nM2KO and WT 24 hrs after MCAO or sham surgery (Red: NeuN; Blue: DAPI; Green: TUNEL). (H, K), Quantification of TUNEL-positive neurons of WT and gM2KO $(\mathrm{H})$, as well as nM2KO and WT sections from 5 mice/group ( ${ }^{* *}, p<0.01$; ANOVA, Bonferroni's t-test, mean \pm SEM). (I, L), Mean percentage of TUNNEL positive neurons in all NeuN positive cells $\left({ }^{* * *}, p<0.001\right.$; ANOVA, Bonferroni's test; mean \pm SEM).

M-P, Evaluation of $\mathrm{Ca}^{2+}$ overload and neuronal death caused by OGD using ratio $\mathrm{Ca}^{2+}$ imaging. Cortical neurons isolated from WT and gM2KO mice were cultured for 7 to 14 days before OGD experiments. (M), Ratio $\mathrm{Ca}^{2+}$ imaging showing intracellular $\mathrm{Ca}^{2+}$ changes induced by OGD in WT and gM2KO neurons. Fluorescence ratio $F_{340 / 380}$ was used to represent intracellular $\mathrm{Ca}^{2+}$ changes (scale bar in bottom left image $=100 \mu \mathrm{m}$ ). Neurons with increasingly elevated $\mathrm{Ca}^{2+}$ levels, such as the ones indicated with arrows, die with time, as reflected by the disappearance of the fluorescence at the next time point. Ionomycin was used to induce the maximum $\mathrm{Ca}^{2+}$ influx for normalization (not shown). (N), Representative real-time changes of $\mathrm{Ca}^{2+}$ induced by OGD in the first 45 min. The averaged traces were from 20 neurons randomly chosen from a representative culture dish of WT and gM2KO groups $\left(^{* * *}, p<0.001\right.$, unpaired $t$-test, mean \pm SEM). (O), Quantification of OGD-induced Fura-2 fluorescence 
changes 30 min after OGD. A cohort of 238 neurons from three WT mice in 6 culture dishes and 233 neurons from gM2KO mice in 6 culture dishes were used for analysis $\left(^{* * *}, p<0.001\right.$, unpaired $t$-test, mean $\left.\pm S E M\right)$. (P), OGD-induced neuronal death at $30 \mathrm{~min}, 60 \mathrm{~min}$, and $90 \mathrm{~min}$ after OGD ${ }^{* * *}, \mathrm{p}<0.001$, unpaired $t$-test, mean \pm SEM, $n=238$ and 233 neurons in WT and gM2KO groups). Neuronal death was monitored as gradually reduced and eventually disappeared $\mathrm{F}_{340 / 380}$ fluorescence after the fluorescence reached maximal level (see representative dead cells indicated by arrows) (ns, $p>0.05$; ${ }^{* *}, p<0.001$; ANOVA, Bonferroni's test; mean \pm SEM). (see also Figure S3 for nM2KO versus WT results).

$(\mathrm{Q}-\mathrm{R})$, Effects of Trpm2 deletion on mitochondrial function of cortical neurons during OGD evaluated by dequenching of R123 fluorescence. (Q), Representative images of R123-labelled mitochondria before and 30 min after OGD in cultured WT and gM2KO cortical neurons. Control group (no OGD treatment) was used to show the rapid photo bleaching of R123. (R), Average changes of R123 fluorescence 30 min after OGD. WT neurons ( $n=74$ for OGD, $n=39$ for control) and gM2KO neurons $(n=123)$ were from 4 dishes of cultured neurons isolated from 3 mice ${ }^{* * *}, p<0.001 ;$ ANOVA, Bonferroni's test; mean \pm SEM).

(S-T), Enhanced TRPM2 function by OGD. TRPM2 currents elicited by a ramp protocol ranging from -100 to +100 $\mathrm{mV}$ in cultured cortical neurons from WT mice using pipette solutions containing $100 \mathrm{nM} \mathrm{Ca}^{2+}$ and $10 \mu \mathrm{M}$ ADPR (S). Averaged current amplitude (measured at $+100 \mathrm{mV}$ ) was increased by 1.4 -fold after OGD (T) $\left(^{*}, p<0.05\right.$, unpaired $t$-test, mean \pm SEM, $\mathrm{n}=7$ ).

(U-V), Up-regulation of TRPM2 by MCAO. TRPM2 expression level analyzed by western blotting (U) was increased by 7.6 -fold $(\mathrm{V})$ in the infarcted (right) hemisphere of brains from MCAO WT mice in comparison with sham-operated WT mice 24 hrs after surgery. Protein levels were normalized by $\beta$-Tubulin $\left({ }^{* \star *}, p<0.001\right.$, unpaired $t$-test, mean \pm SEM, $\mathrm{n}=12 /$ group) .

(W-Z), Deletion of TRPM2 abolishes the increase of surface expression of NMDARs induced by MCAO. (W), Representative western blotting of the surface expression of GluN1a, GluN2a, and GluN2b in cell membrane protein extractions of 3 brains from WT and gM2KO mice subjected to either sham surgery or MCAO. (X-Z), changes of surface expression levels of GluN1a, GluN2a, and GluN2b in WT and gM2KO mice subjected to either sham surgery or MCAO. The entire hemisphere at the operation side (right hemisphere) was harvested 24 hrs after surgery for protein extraction. Protein levels were normalized to the membrane protein loading control pancadherin for quantification (ns, no statistical significance, ${ }^{*}, p<0.05,{ }^{* *}, p<0.01,{ }^{* * *}, p<0.001$; ANOVA, Bonferroni's test; mean \pm SEM, $n=12$ ). 
(A-B), Co-immunoprecipitation (Co-IP) of NMDARs and TRPM2 expressed in HEK-293T cells. NMDAR subunits, GluN1a, GluN2A, and GluN2B were transfected with Flag-tagged TRPM2 (Flag-TRPM2) or EGFP empty vector plasmids. (A), TRPM2 was immunoprecipitated (IP'd) using anti-GluN1a, anti-GluN2a and anti-GluN2b agarose, and detected using western blotting (WB) with anti-Flag. (B), Cell lysates were IP'd using anti-Flag agarose and were probed using WBs with anti-GluN1a, anti-GluN2a, and anti-GluN2b. All the transfections, IP, and WB were replicated 3 times.

(C), Co-IP of each subunit of NMDARs and TRPM2 in HEK-293T cells in which Flag-TRPM2 was co-transfected with GluN1a (TRPM2/GluN1a), GluN2a (TRPM2/GluN2a), or GluN2b (TRPM2/GluN2b). EGFP plasmid was used as a control for transfection with each subunit of NMDARs. Cell lysates were IP'd using anti-TRPM2 agarose and were probed using WBs with anti-GluN1a, anti-GluN2a, or anti-GluN2b. TRPM2 interacted with GluN2a and GluN2b, but not GluN1a. All the transfections, Co-IP, and WB were replicated 3 times.

(D-E), Endogenous TRPM2 and NMDARs interaction. Co-IP of NMDARs and TRPM2 using protein extractions from brains of WT mice after MCAO. Brain extracts of gM2KO mice subjected to MCAO were used as control. (D) Brain lysates were IP'd using anti-GluN1a, anti-GluN2a, and anti-GluN2b agarose and were probed using WB with anti-TRPM2. (E) Brain lysates were Co-IP'd using anti-TRPM2 antibody and were probed using WBs with GluN1a, GluN2a, and GluN2b. All Co-IP and WB were replicated using at least 3 mouse brains.

(F), Representative NMDAR currents recorded by holding at $-80 \mathrm{mV}$ in cortical neurons cultured for 14 days from WT and gM2KO mice. NMDA at $10 \mu \mathrm{M}$ was applied for $\sim 5$ to $10 \mathrm{~s}$. Average peak current amplitude of NMDARs from WT and gM2KO neurons is shown in inset $\left({ }^{* * *}, p<0.001\right.$, unpaired $t$-test, mean $\pm S E M, n=20 /$ group).

(G-J), Surface expression changes of NMDARs in HEK-293T cells co-transfected with TRPM2 (NMDARs/TRPM2, or “+M2"), or EGFP plasmid as control (NMDARs/EGFP, or "Con"). (G), Membrane (Mem) and cytosol (Cyto) protein levels assessed with WBs. Pan-cadherin and $\beta$-tubulin were used as loading control for membrane and cytosol proteins extracts respectively. (H-J), Quantification of the expression of GluN1a, GluN2a, and GluN2b in cell membrane and cytosol $\left({ }^{*}, p<0.05,{ }^{* *}, p<0.01\right.$, unpaired $t$-test, mean $\left.\pm \mathrm{SEM}\right)$. All transfections, extraction of membrane/cytosolic proteins and immunoblotting were repeated at least 3 times.

(K-L), Functional changes of NMDARs when co-expressed with TRPM2 in HEK293T cells. (K), Representative NMDAR currents recorded by holding at $-80 \mathrm{mV}$ in HEK293T cells transfected with NMDARs/TRPM2, or NMDARs/EGFP. NMDA at $10 \mu \mathrm{M}$ was applied for $\sim 5$ to $10 \mathrm{~s}$ to activate NMDARs. (L), Average peak current amplitude from NMDARs/EGFP group $(n=23)$ and NMDARs/TRPM2 group $(n=27)\left({ }^{* * *}, p<0.001\right.$, unpaired $t$-test, mean \pm SEM). 
Figure 3 | Identification of the interacting domain $\mathrm{EE}_{3}$ in the $\mathrm{N}$-terminus of TRPM2 that mediates the

\section{physical and functional coupling between TRPM2 and NMDARs}

(A-B), Co-IP of N-terminal (1-727) and C-terminal (1060-1503) fragments of TRPM2 (TRPM2-NT, TRPM2-CT) with NMDARs in the HEK293T cells co-transfected with NMDARs or EGFP plasmids. TRPM2-NT (A) and TRPM2CT (B) were IP'd using anti-GluN1a, anti-GluN2a, or anti-GluN2b antibodies and were probed by WBs using antiflag (A) or anti-GFP (B). TRPM2-NT was flag-tagged and TRPM2-CT was GFP-tagged. (A), TRPM2-N interacted with NMDARs, as an estimated $85 \mathrm{kDa}$ fragment was detected by anti-flag in NMDARs/TRPM2-N co-transfected cells but not in the NMDARs/EGFP-transfected cells. (B), TRPM2-CT did not interact with NMDARs as the estimated $60 \mathrm{kDa}$ fragment of TRPM2-CT was only detected by anti-GFP in the input of NMDARs/TRPM2-CT cotransfected cell. These results were replicated in three independent experiments.

(C), Co-IP of TRPM2 with the C-terminus of GluN2a (GluN2a-CT, 1054-1068), C-terminus deleted GluN2a (GluN2a- $\Delta$ C, 1-1053), C-terminus of GluN2b (GluN2b-CT, 1041-1691), and C-terminus deleted GluN2b (GluN2b$\Delta \mathrm{C}, 1-1047)$ over-expressed in the HEK293T cells. All the constructs were GFP-tagged. GluN2a-CT, GluN2b-CT, GluN2a- $\Delta \mathrm{CT}$, and GluN2b- $\Delta$ CT were IP'd using anti-TRPM2 and were probed using WB by anti-GFP. Note that only the GluN2a-CT and GluN2b-CT interacted with TRPM2. These results were replicated in three independent experiments.

(D), Representative NMDAR currents recorded in the HEK-293T cells transfected with NMDARs/EGFP, NMDARs/TRPM2-full length (FL), NMDARs/TRPM2-NT, and NMDARs/TRPM2-CT (Left). NMDAR currents were elicited by $10 \mu \mathrm{M}$ NMDA in tyrode solution perfused for about 7 to $10 \mathrm{~s}$ at $-80 \mathrm{mV}$. Average current amplitudes of NMDARs/EGFP group $(n=9)$, NMDARs/TRPM2-FL group $(n=8)$, NMDARs/TRPM2-NT group $(n=8)$, and NMDARs/TRPM2-CT group $(n=8)$ are shown in the bar graph at the right side ( $n s, p>0.05 ;{ }^{* * *}, p<0.001$; ANOVA, Bonferroni's test; mean \pm SEM).

(E), NMDARs-mediated $\mathrm{Ca}^{2+}$ influx in HEK-293T cells transfected with NMDARs/EGFP, NMDARs/TRPM2-FL,

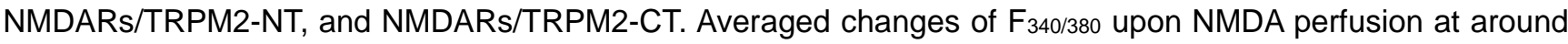
15 to $25 \mathrm{~s}$ (left), and the mean $\Delta \mathrm{F}_{340 / 380}$ measured at $\sim 40 \mathrm{~s}$ is shown in the bar graph (right). (ns, $\mathrm{p}>0.05 ;{ }^{* *}, \mathrm{p}<$ 0.01; ANOVA, Bonferroni's test; mean \pm SEM; $n=17,14,13$, and 16, respectively, from 3 testing dishes/group). (F), Schematic diagram of the membrane topology of TRPM2. The $\mathrm{EE}_{3}$ domain is localized within the MHR4. (G-L), Identification of NMDAR-binding domain at the TRPM2 N-terminus. Co-IP of Flag-TRPM2 N-terminal 
segments with different lengths (1-570, 1-631, 1-678 and 1-727) with NMDARs co-transfected in HEK-293T cells. $(G)$, lysates were IP'd using anti-TRPM2-N (against TRPM2 N-terminus) and were probed by WBs with antiGluN2a or anti-GluN2b. TRPM2-CT and TRPM2-FL were included as negative and positive control, respectively. $(\mathrm{H}, \mathrm{I})$ Different fragments of Flag-TRPM2 were IP'd with anti-GluN2A $(\mathrm{H})$ or GluN2b $(\mathrm{I})$ and were probed by WBs with anti-Flag. Fragments shorter than 631 aa failed to interact with NMDARs. The results were replicated at least three times.

$(\mathrm{J}-\mathrm{L})$, Identification of the $\mathrm{EE}_{3}$ domain as the binding site for TRPM2 and NMDARs. Co-IP of the TRPM2 Nterminal fragments (1-664 and 1-679) with NMDARs co-expressed in the HEK293T cells. (J), GluN2a and Glu2N2b were IP'd with N-terminal anti-TRPM2 and probed using WBs with anti-GluN2a and GluN2b. (K, L), TRPM2 N terminal segments (1-664 and 1-679) were IP'd with anti-GluN2a (K) and anti-GluN2b, and were probed using WBs with anti-Flag. Fragment 1-664 failed to interact with NMDARs. The critical residues 665-679 for physical interaction together with additional "EE" at the position 680-681 was defined as the $\mathrm{EE}_{3}$ binding domain. The results were replicated in three independent experiments.

(M-P), Physical and functional coupling of TRPM2 and NMDARs through $\mathrm{EE}_{3}$ domain. (M, O), $\mathrm{EE}_{3}$ domain deletion mutant of TRPM2 (TRPM2- $\triangle \mathrm{EE}_{3}$ ), and EE $\mathrm{E}_{3}$ mutations of TRPM2, TRPM2-QEE (E666Q and E667Q), TRPM2EQE (E673Q and E674Q), and TRPM2-EEQ (E680Q and E681Q) were co-expressed with NMDARs in HEK293T

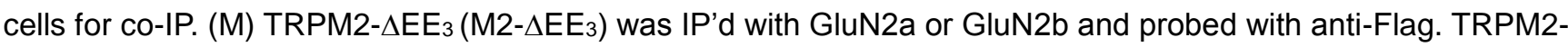
WT (M2-WT) was used as control. (O), TRPM2 mutants QEE, EQE, and EEQ were IP'd with anti-TRPM2 and probed with GluN2a or GluN2b. WT-TRPM2 (EEE) was used as a control. (N), NMDAR currents elicited by NMDA in HEK293T cells co-expressed with empty EGFP vector, WT-TRPM2, TRPM2- $\triangle E^{E} E_{3}$, TRPM2-QEE, TRPM2-EQE, and TRPM2-EEQ. (P), Mean current amplitude (ns, $p<0.05 ;{ }^{* * *}, p<0.001$; ANOVA, Bonferroni's test; mean \pm SEM, $n=10$ /group). 


\section{Figure 4 | N-terminal domain of TRPM2 Interacts with PKC- $\gamma$}

(A-B), Co-IP of PKC $\gamma$ and TRPM2 using proteins extracted from mouse brains after MCAO (A), and in HEK293T cells expressing PKC $\gamma$ with TRPM2-FL, TRPM2-NT, or TRPM2-CT (B). PKC- $\gamma$ was IP'd with anti-TRPM2 and was probed by WBs using anti-PKCY. The results were replicated in 3 independent experiments.

(C,D), Oxidative stress promotes TRPM2 and PKC $\gamma$ interaction in MCAO brains. (C), Co-IP of TRPM2 and PKC $\gamma$ using protein extracts from WT mice brains subjected to MCAO or sham operation. PKC- $y$ was IP'd using antiTRPM2 and was probed with anti-PKC $\gamma$ by WB. TRPM2 was used as control for IP protein, and $\beta$-tubulin was used as loading control. (D) Mean PKC $\gamma / T R P M 2$ ratios in sham and MCAO mice. ( ${ }^{* \star *}, \mathrm{p}<0.001$, unpaired $t$-test, mean \pm SEM; $n=6)$.

(E,F), Effects of $\mathrm{H}_{2} \mathrm{O}_{2}$ on TRPM2 and PKC $\gamma$ interaction in cultured neurons. (E), PKC $\gamma$ was IP'd with anti-TRPM2 in protein extracts from cultured WT cortical neurons exposed to $\mathrm{H}_{2} \mathrm{O}_{2}$ (at $100 \mu \mathrm{M}$ for 1 min, $3 \mathrm{~min}$, and 5 min, at $300 \mu \mathrm{M}$ for $5 \mathrm{~min}$ and at $1 \mathrm{mM}$ for $5 \mathrm{~min}$ ), and probed with anti-PKC- $\gamma$. For IP proteins, TRPM2 was used as loading control. For lysates, $\beta$-tubulin was used as loading control. (F), Average PKC $\gamma$ normalized to TRPM2 from 3 independent experiments. ${ }^{* * *}, p<0.001$; ANOVA, Bonferroni's test; mean $\pm S E M, n=3 /$ group).

(G-J), Surface expression of NMDARs in HEK-293T cells co-transfected with PKC-y/EGFP (Con) and PKCY/TRPM2 (+M2) (G) or PKC-y-DN/EGFP (Con) and PKC-y-DN/TRPM2 (+M2) (I). Membrane and cytosol protein levels of NMDARs were quantified from 3 independent experiments $(H, J) .\left({ }^{*}, p<0.05,{ }^{* *}, p<0.01,{ }^{* * *}, p<0.001\right.$, unpaired $t$-test, mean \pm SEM).

(K-N), Surface expression of NMDARs in HEK-293T cells co-transfected with TRPM2 or EGFP with the treatment of PKC activator PMA (K) or inhibitor Staurosporine (M). Membrane (Mem) and cytosol (Cyto) NMDARs were quantified from 3 independent experiments $(\mathrm{L}, \mathrm{N}) .\left({ }^{*}, \mathrm{p}<0.05,{ }^{* *}, \mathrm{p}<0.01,{ }^{* * *}, \mathrm{p}<0.001\right.$, unpaired $t$-test, mean \pm SEM, $n=3$ /group).

$(\mathrm{O}, \mathrm{P})$, Effects of staurosporine on NMDAR currents recorded from cortical neurons cultured for 14 days. (O), Representative NMDAR currents elicited at $-80 \mathrm{mV}$ by $10 \mu \mathrm{M}$ NMDA in WT and TRPM2-KO (gM2KO) neurons treated with or without Staurosporine at $1 \mu \mathrm{M}$ for overnight. $(P)$, Mean current amplitude (ns, $p>0.05 ;{ }^{* *}, p<0.01$; ANOVA, Bonferroni's test; mean \pm SEM, $n=11,10,10$, and 12 neurons from 2 mice, respectively).

$(\mathrm{Q}-\mathrm{T})$, Effects of Endosidin2 on NMDA currents recorded from cortical neurons cultured for 14 days. (S) Representative NMDAR currents elicited by NMDA in WT and TRPM2-KO neurons treated with or without endosidin2 at $1 \mu \mathrm{M}$ overnight. (T) Average current amplitude (ns, p>0.05; ${ }^{*}, \mathrm{p}<0.05$; ANOVA, Bonferroni's test; mean $\pm S E M, n=10$ neurons/group from 2 mice respectively). 


\section{Figure 5 | TAT-EE 3 disrupts the physical and functional interaction between TRPM2 and NMDAR}

(A-B), Effects of TAT-EE 3 on the interaction of TRPM2 and NMDARs. Co-IP tests of NMDARs with WT or EE ${ }_{3-}$ domain mutants of TRPM2 (Flag-tagged) in co-transfected HEK293T cells. WT-TRPM2 groups were treated with $10 \mu \mathrm{M}$ TAT-SC or TAT-EE 3 overnight. Lysates were IP'd with anti-GluN2a (A) or anti-GluN2b (B), and probed by WBs with anti-Flag. IgG was used as control for IP. Similar to the mutants TRPM2- $\triangle \mathrm{EE}_{3}$ and TRPM2-EQE, TAT$\mathrm{EE}_{3}$ disrupted TRPM2 interactions with GluN2a and GluN2b. These results were replicated in 3 independent experiments.

(C-F), Effects of TAT-EE3 on surface expression of NMDARs. HEK293T cells co-transfected with NMDARs and TRPM2 (+M2) or EGFP vector (Con) were treated with $10 \mu \mathrm{M}$ TAT-SC (C) or TAT-EE 3 (D) overnight. Membrane (Mem) and cytosol (Cyto) proteins of NMDARs in TAT-SC (E) and TAT-EE 3 (F) treated groups were assessed by WBs and analyzed in reference to Pan-cadherin or $\beta$-tubulin ${ }^{* *}, p<0.01,{ }^{* * *}, p<0.001$, unpaired $t$-test, mean \pm SEM). All the results were replicated in at least 3 independent experiments.

$(G-H)$, Effects of TAT-EE 3 on NMDAR currents in HEK-293T cells transfected with NMDARs and TRPM2. Transfected cells were treated with $10 \mu \mathrm{M}$ TAT-EE 3 or TAT-SC for overnight. (G), Representative NMDAR currents elicited at $-80 \mathrm{mV}$ by exposing to $10 \mu \mathrm{M}$ NMDA for about 2 to $4 \mathrm{~s}$. $(\mathrm{H})$, Mean current amplitude of NMDARs/EGFP $(n=13)$, and NMDARs/TRPM2 treated with TAT-SC $(n=17)$ or TAT-EE3 $(n=16)$ groups $\left(n s, p>0.05 ;{ }^{* * *}, p<0.001\right.$; ANOVA, Bonferroni's test; mean \pm SEM).

(I-K), Effects of TAT-EE 3 on PKC-induced changes of NMDAR currents recorded in cortical neurons of WT and TRPM2-KO. Neurons were incubated with $10 \mu \mathrm{M}$ TAT-SC or TAT-EE 3 overnight before current recording. (I) Representative NMDAR currents elicited by $10 \mu \mathrm{M}$ NMDA at $-80 \mathrm{mV}$ before and after PMA $(1 \mu \mathrm{M})$ perfusion for $20 \mathrm{~s}$. TAT-SC or TAT-EE3 $10 \mu \mathrm{M}$ was included in the pipette solution for current recording. (J), Average NMDAR current amplitude before and after $1 \mu \mathrm{M}$ PMA from WT and TRPM2-KO neurons treated with TAT-SC or TAT-EE 3. (K), Average percentage increases of NMDAR currents induced by PMA in WT and TRPM2-KO neurons treated with TAT-SC or TAT-EE 3 ( $n s,{ }^{*} p>0.05 ;{ }^{* * *}, p<0.001$; ANOVA, Bonferroni's test; mean \pm SEM, $n=19,14,14$ and 11 , respectively).

Figure 6 | Uncoupling TRPM2 and NMDARs by TAT-EE $E_{3}$ protects neurons against ischemic injury in vitro and in vivo by preserving pro-survival signaling

(A-B), Effects of TAT-EE 3 on NMDAR currents in WT and gM2KO cortical neurons cultured for 14 days. (A), Representative recording of NMDAR currents in cultured neurons treated with TAT-SC or TAT-EE3 at $10 \mu \mathrm{M}$ 
overnight before current recording. TAT-SC or TAT-EE3 $10 \mu \mathrm{M}$ was included in the pipette solution during current recording. (B), Average current amplitude in WT and gM2KO neurons treated with TAT-SC or TAT-EE 3 (ns, p>0.05; ${ }^{* * *}, p<0.001 ;$ ANOVA, Bonferroni's test; mean \pm SEM, $n=15,13,12$, and 14, respectively).

(C-E), Effects of TAT-EE 3 on $\mathrm{Ca}^{2+}$ overload and neuronal death caused by OGD. Cultured WT and gM2KO cortical neurons were treated with $10 \mu \mathrm{M}$ TAT-SC and TAT-EE3 overnight before experiments. (C), Ratio $\mathrm{Ca}^{2+}$ imaging showing intracellular $\mathrm{Ca}^{2+}$ changes induced by OGD in TAT-EE3- or TAT-SC-treated WT and gM2KO neurons. Fluorescence ratio $\mathrm{F}_{340 / 380}$ was used to represent intracellular $\mathrm{Ca}^{2+}$ changes (see the scale bar at the right shoulder of panel C). Neurons with increasingly elevated $\mathrm{Ca}^{2+}$ levels such as the ones indicated with arrows die with time, as reflected by the disappearance of the fluorescence at the next time point. Ionomycin (lono) was used at the end of the experiments to serve as an internal control for normalizing $F_{340 / 380}$ (not shown). (D), $\mathrm{Ca}^{2+}$ increases represented by normalized $F_{340 / 380}$ induced by $O G D$ in the first 45 min analyzed from 20 neurons randomly chosen from each group. (E), Average neuronal death rate (see panel (C)) evaluated at 30,60, and 90 min after OGD. Neuronal death was monitored as F340/380 fluorescence gradually reduced and eventually disappeared after the fluorescence reached maximal level (see representative dead cells indicated by arrows in (C)) (ns, $p>0.05 ;{ }^{* \star *}, p$ $<0.001$; ANOVA, Bonferroni's test; mean \pm SEM).

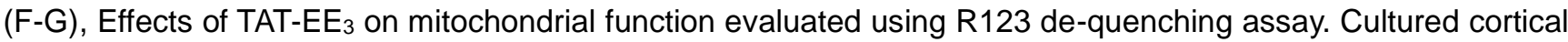
neurons from gM2KO mice, nM2KO mice, and WT control littermates were incubated with $10 \mu \mathrm{M}$ TAT-SC or TATEE3 for overnight before OGD exposure. (F), R123 fluorescence changes in neurons of different groups induced by OGD. External perfusion solution aCSF containing no $\mathrm{Ca}^{2+}\left(\mathrm{No} \mathrm{Ca}^{2+}\right)$ was used as a control. WT neurons without OGD exposure were used as a control (WT control) to illustrate the rapid photo bleaching of Rh123. (G), Mean R123 fluorescence changes induced by 30 min OGD exposure in TAT-SC ( $n=682)$, TAT-EE $3(n=499)$, Global M2KO ( $n=374)$, neuronal M2KO (549) neurons, or no-OGD Control (=258). The accumulated numbers of neurons in each group for data analysis were from 3 5 independent experiments using neurons isolated from 3 mice/group $\left({ }^{* * *}, p<0.001 ;\right.$ ANOVA, Bonferroni's test; mean \pm SEM $)$.

$(H-J)$, TAT-EE $E_{3}$ reduces infarct size and improves neurological deficit (ND) score in WT MCAO mice. $(H)$, TTC staining of brain slices $24 \mathrm{hrs}$ after MCAO from WT mice intraperitoneally (ip) administrated with TAT-SC or TAT$\mathrm{EE}_{3}$ at $100 \mathrm{nmol} / \mathrm{kg} 15 \mathrm{~min}$ before MCAO procedure. (i), Mean infarct volume after MCAO. (J), Average neurological deficit score. ${ }^{* * *}, p<0.001$; ANOVA, Bonferroni's test; mean $\pm S E M ; n=7$ for TAT-SC and $n=8$ for TAT-EEз groups).

(K-L), Effects of TAT-EE $E_{3}$ on surface expression of NMDARs in WT and nM2KO mice 24 hrs after MCAO or sham procedure. TAT-SC and TAT-EE 3 were ip administrated $(100 \mathrm{nmol} / \mathrm{kg}) 15 \mathrm{mins}$ before MCAO or sham procedure. 
(K) WB of surface expression of NMDARs from two representative samples/group. Pan-cadherin was used as loading control. (I), Quantification of surface expression of NMDARs normalized to Pan-cadherin (ns, p>0.05; ***, $p<0.001 ;$ ANOVA, Bonferroni's test; mean \pm SEM; $n=4$ mice/group).

(M-O), Effects of TAT-EE 3 on ERK1/2 and CREB activities in cultured neurons isolated from gM2KO and nM2KO mice, and WT littermates. WT neurons were treated with TAT-EE 3 or TAT-SC overnight before the experiments. Neurons were incubated for $1 \mathrm{~h}$ before experiments with 1) control (PBS), 2) NMDA (10 $\mu \mathrm{M})$ to activate synaptic and extrasynaptic NMDARs, and 3) 4-AP $2.5 \mathrm{mM}$ plus bicuculine (Bic) $50 \mu \mathrm{M}$ (4-AP/Bic) to activate synaptic NMDARs. (M), ERK1/2 and CREB activities were evaluated by detecting phosphorylated ERK1/2 (p4/42ERK1/2) and CREB (pSer ${ }^{133} \mathrm{CREB}$ ). (N-O), Quantification of the active CERB and ERK1/2 in each group under three different treatment conditions $\left(^{* * *}, p<0.001\right.$; ANOVA, Bonferroni's test; mean $\left.\pm S E M, n=3\right)$.

(Q-R), Effects of TAT-EE 3 and Trpm2 deletion on ERK and CREB activities in mice subjected to MCAO. (P), ERK and CREB activities were evaluated by assessing the levels of pERK1/2 and pCREB in nM2KO mice and WT control littermates subjected to MCAO or sham surgery. TAT-EE 3 or TAT-SC was ip administrated to WT mice 15 min before $M C A O$ or sham surgeries. $(Q, R)$, Quantification of $p E R K 1 / 2$ and $p C R E B$ expressed in the brains after MCAO or sham procedure $\left({ }^{* * *}, p<0.001\right.$; ANOVA, Bonferroni's test; mean \pm SEM, $n=4 /$ group $)$.

\section{Supplementary Figure 1 | TRPM2 currents recorded in WT neurons and determination of TRPM2 deletion}

\section{in the global and neuron-specific knockout mice.}

$(A-E)$, TRPM2 currents recorded in the cortical neurons with pipette solution containing ADPR and $\mathrm{Ca}^{2+}$. (A, C), Representative currents elicited by a ramp protocol ranging from -100 to $+100 \mathrm{mV}$ in WT neurons (A) but not in global TRPM2-KO (gM2KO) neurons (C). NMDG was used to ensure no leak contamination, and ACA (30 $\mu \mathrm{M})$ was used to inhibit TRPM2 currents. (B, D), Inward and outward current measured at $-100 \mathrm{mV}$ and $+100 \mathrm{mV}$ were plotted against time (B). No currents were recorded in the gM2KO neurons (D). (E), Average current amplitude (at $+100 \mathrm{mV}$ ) of TRPM2 in WT neurons; TRPM2 current was eliminated in gM2KO neurons. Please note that NMDG eliminated inward TRPM2 currents (A, B), indicating no leak current, but meanwhile slightly reduced outward currents $(\mathrm{A}, \mathrm{B})$ because elimination of extracellular $\mathrm{Ca}^{2+}$ entry will gradually close TRPM2.

(F-G), Conformation of global TRPM2 knockout (gM2KO) by genotyping (G) and WB (F). (F), Representative WB results from 3 brains of WT and gM2KO mice. (G), Representative PCR genotyping results showing a 514 bp and 740 bp products for WT and gM2KO mice.

$(\mathrm{H}-\mathrm{I})$, Representative TRPM2 currents recorded in neurons from WT and neuron-specific TRPM2-KO (nM2KO) 
mice. TRPM2 currents were recorded in cortical neurons from WT neurons but not in nM2KO neurons $(H)$. Average currents measured at $+100 \mathrm{mV}$ (I). Please note that nM2KO eliminated TRPM2 currents.

(J-K), Conformation of neuron-specific knockout of TRPM2 by WB and genotyping. (J), Representative WB results to detect TRPM2 deletion using cultured neurons from 3 WT and nestin-cre+ floxed mice (nM2KO). TRPM2 protein was largely eliminated in cultured neurons of nM2KO. The trace amounts of protein detected in M2KO neuron cultures is likely from non-neuronal cells in the culture dishes. (K), Representative PCR results for genotyping of TRPM2 flox/flox expression. The predicted PCR products are 400 bp in TRPM2-flox/flox expressing mice ( $n-6)$ and $260 \mathrm{bp}$ in a WT mouse as control.

\section{Supplementary Figure 2| Blood flow changes measured using Laser Doppler Flowmetry (LDF)}

(A-B), Representative data of blood flow changes measured using LDF. Blood flow was measured using LDF before and after MCAO, as well as after reperfusion. Successful MCA occlusion was confirmed by $85 \%$ reduction of cerebral blood flow OGD ${ }^{* * *}, \mathrm{p}<0.001$, unpaired $t$-test, mean \pm SEM; $\mathrm{n}=18$ for WT and $\mathrm{n}=16$ for gM2KO groups; and $\mathrm{n}=13$ for WT and $\mathrm{n}=11$ for nM2KO groups).

\section{Supplementary Figure 3 $\mid$ Neuron-specific Trpm2 Knockout (nM2KO) protects neurons from oxygen-}

\section{glucose deprivation (OGD)-induced damage}

(A), Evaluation of $\mathrm{Ca}^{2+}$ overload and neuronal death using Fura-2 real-time ratio $\mathrm{Ca}^{2+}$ imaging. Cortical neurons were isolated from nM2KO mice $\left(\right.$ Trpm2 $\left.2^{\text {floxflox }}, \mathrm{Cre}^{+}\right)$and WT littermate control mice (Trpm2 $\left.2^{\text {floxfllox }}, \mathrm{Cre}^{-}\right)$and cultured for 7 to 14 days. Neurons were exposed to OGD and intracellular $\mathrm{Ca}^{2+}$ change was monitored by Fura-2 ratio $\mathrm{Ca}^{2+}$ imaging for 90 mins. Neurons with increasingly elevated $\mathrm{Ca}^{2+}$ levels, such as the ones indicated by arrows, died and disappeared at different time points. Ionomycin was used to induce the maximum $\mathrm{Ca}^{2+}$ influx for normalization (not shown).

(B), Representative sample traces of Fura-2 real-time $\mathrm{Ca}^{2+}$ imaging normalized to ionomycin-induced responses. Averaged traces from 20 neurons which were randomly chosen from WT and nM2KO groups for analysis. Ionomycin was used to induce the maximum $\mathrm{Ca}^{2+}$ influx for normalization $\left({ }^{* * *}, \mathrm{p}<0.001\right.$, unpaired $t$-test, mean \pm SEM).

(C), Quantification of OGD-induced $\mathrm{Ca}^{2+}$ changes after OGD for $30 \mathrm{~min} .238$ neurons from 3 WT mice in 6 culture dishes and 233 neurons from nM2KO mice in 6 culture dishes were used for analysis ${ }^{\star \star \star}, \mathrm{p}<0.001$, unpaired $t$ - 
test, mean \pm SEM).

(D), Quantification of OGD-induced neuronal death at 30,60, and 90 min after OGD ${ }^{\star * *}, \mathrm{p}<0.001$, unpaired $t$ test, mean \pm SEM). Neuronal death was monitored as $F_{340 / 380}$ fluorescence gradually reduced and eventually disappeared after the fluorescence reached maximal level (see representative dead cells indicated by arrows in $(A))$.

\section{Supplementary Figure 4| TRPM2 potentiates both GluN1a/GluN2a and GluN1a/GluN2b surface}

\section{expression levels}

(A-C), Surface expression of GluN1a and GluN2a in HEK293T cells co-expressing EGFP (Con) or TRPM2 (+M2). (A), WB analysis of membrane and cytosol levels of GLuN1a and Glu2a. Pan-cadherin and $\beta$-tubulin were used as loading controls. (B, C), Quantification of GluN1a, GluN2a membrane (Mem) and cytosol (Cyto) expression (*, $\mathrm{p}<0.05,{ }^{* *}, \mathrm{p}<0.01 ;$ unpaired $t$-test, mean $\pm \mathrm{SEM}, \mathrm{n}=3$ ).

(D-F) Surface expression of GluN1a and GluN2b in HEK293T cells co-expressing EGFP (Con) or TRPM2 (+M2). (D), WB analysis of membrane and cytosol levels of GluN1a and GluN2b. Pan-cadherin and $\beta$-tubulin were used as loading control. (E, F), Quantification of GluN1a, GluN2b membrane (Mem) and cytosol (Cyto) expression (*, $\mathrm{p}<0.05,{ }^{* *}, \mathrm{p}<0.01 ;$ unpaired $t$-test, mean $\pm \mathrm{SEM}, \mathrm{n}=3$ ).

(G, H) Representative GluN1a/GluN2a currents (G) recorded in HEK-293T cells co-expressing GluN1a/GluN2a/EGFP or GluN1a/GluN2a/TRPM2, and mean current amplitude $(\mathrm{H}){ }^{* * *}, \mathrm{p}<0.001$; ANOVA, Bonferroni's test; mean \pm SEM, $n=11 \sim 12$ ).

(I, J) Representative GluN1a/GluN2b currents (I) recorded in HEK-293T cells co-expressing GluN1a/GluN2a/EGFP or GluN1a/GluN2a/TRPM2, and mean current amplitude $(\mathrm{J}){ }^{* * *}, \mathrm{p}<0.001$; ANOVA, Bonferroni's test; mean \pm SEM, $n=11 \sim 12$ ).

\section{Supplementary Figure $5 \mid$ Alignment of triple glutamate-glutamate repeats $\left(\mathrm{EE}_{3}\right)$ and functional} evaluation of TRPM2-EE 3 domain mutants

(A, B) Alignment of triple $E E$ domain $\left(E_{3}\right)$ in TRPM subfamily $(A)$, and $E_{3}$ domain in TRPM2 of different species (B).

(C-G) Representative TRPM2 current recordings from HEK-293T cells transfected with $\mathrm{EE}_{3}$ domain deleted TRPM2 (TRPM2- $\triangle \mathrm{EE}_{3}$ ) and TRPM2 mutants (QEE: E666Q, E667Q; EQE: E673Q, E674Q; EEQ: E680Q, E681Q). 
(H) Average current quantification of sample traces of TRPM2 current recording from HEK-293T cells transfected with different TRPM2 mutants. 10 recordings from each group were used for analysis (ns, $p>0.05$; ANOVA, Bonferroni's test; mean \pm SEM).

\section{Supplementary Figure 6 | Effects of endosidin2 on surface expression levels of NMDARs.}

(A), Surface expression of GluN1a, GluN2a, and GluN2b was detected by WB in HEK-293T cells transfected with NMDARs/EGFP (Con) and NMDARs/TRPM2 (+M2) after incubation with $1 \mu \mathrm{M}$ endosidin2 for overnight. Pancadherin and $\beta$-tubulin were used as loading control for membrane and cytosolic protein extraction.

(B), Quantification of the expression of GluN1a, GluN2a, and GluN2b in cell membrane (Mem) and cytosol (Cyto) (ns, $\mathrm{p}>0.05$; unpaired $t$-test; mean \pm SEM, $\mathrm{n}=3$ ).

\section{Supplementary Figure 7 | Global TRPM2 Knockout preserves CREB and ERK-1/2 signaling after MCAO}

(A), Western blotting analysis of changes of p-ERK 1/2, ERK-1/2, p-CREB, and CREB expression in the brain from WT and TRPM2-KO subjected to sham or MCAO surgery.

(B-C), Quantification of $p E R K-1 / 2$ and pCREB after MCAO or sham surgery. Four mice from each group were used for quantification ( ${ }^{* *}, p<0.01$; ANOVA, Bonferroni's test; mean \pm SEM, $n=4 /$ group). 


\section{REFERENCES}

Abramov, A.Y., and Duchen, M.R. (2008). Mechanisms underlying the loss of mitochondrial membrane potential in glutamate excitotoxicity. Biochimica et biophysica acta 1777, 953-964.

Alim, I., Teves, L., Li, R., Mori, Y., and Tymianski, M. (2013). Modulation of NMDAR subunit expression by TRPM2 channels regulates neuronal vulnerability to ischemic cell death. The Journal of neuroscience : the official journal of the Society for Neuroscience 33, 17264-17277.

Bading, H. (2013). Nuclear calcium signalling in the regulation of brain function. Nature reviews Neuroscience $14,593-608$.

Bayes, A., Collins, M.O., Croning, M.D., van de Lagemaat, L.N., Choudhary, J.S., and Grant, S.G. (2012). Comparative study of human and mouse postsynaptic proteomes finds high compositional conservation and abundance differences for key synaptic proteins. PloS one 7, e46683.

Belrose, J.C., and Jackson, M.F. (2018). TRPM2: a candidate therapeutic target for treating neurological diseases. Acta pharmacologica Sinica 39, 722-732.

Belrose, J.C., Xie, Y.F., Gierszewski, L.J., MacDonald, J.F., and Jackson, M.F. (2012). Loss of glutathione homeostasis associated with neuronal senescence facilitates TRPM2 channel activation in cultured hippocampal pyramidal neurons. Mol Brain 5, 11.

Carroll, R.C., and Zukin, R.S. (2002). NMDA-receptor trafficking and targeting: implications for synaptic transmission and plasticity. Trends in neurosciences 25, 571-577.

Chiang, T., Messing, R.O., and Chou, W.H. (2011). Mouse model of middle cerebral artery occlusion. Journal of visualized experiments : JoVE.

Choi, D.W. (2020). Excitotoxicity: Still Hammering the Ischemic Brain in 2020. Frontiers in neuroscience 14, 579953.

Clapham, D.E. (2003). TRP channels as cellular sensors. Nature 426, 517-524.

Csanady, L., and Torocsik, B. (2009). Four Ca2+ Ions Activate TRPM2 Channels by Binding in Deep Crevices near the Pore but Intracellularly of the Gate. J Gen Physiol 133, 189-203.

Du, J., Xie, J., and Yue, L. (2009a). Intracellular calcium activates TRPM2 and its alternative spliced isoforms.

Proceedings of the National Academy of Sciences 107, 7239-7244.

$\mathrm{Du}, \mathrm{J} ., \mathrm{Xie}, \mathrm{J}$. , and Yue, L. (2009b). Modulation of TRPM2 by acidic $\mathrm{pH}$ and the underlying mechanisms for $\mathrm{pH}$ sensitivity. J Gen Physiol 134, 471-488.

Du, J., Xie, J., Zhang, Z., Tsujikawa, H., Fusco, D., Silverman, D., Liang, B., and Yue, L. (2010). TRPM7mediated $\mathrm{Ca}^{2+}$ signals confer fibrogenesis in human atrial fibrillation. Circulation research 106, 992-1003.

Fonfria, E., Murdock, P.R., Cusdin, F.S., Benham, C.D., Kelsell, R.E., and McNulty, S. (2006). Tissue distribution profiles of the human TRPM cation channel family. Journal of receptor and signal transduction research 26, 159-178.

Ge, Y., Chen, W., Axerio-Cilies, P., and Wang, Y.T. (2020). NMDARs in Cell Survival and Death: Implications in Stroke Pathogenesis and Treatment. Trends in molecular medicine 26, 533-551.

Gelderblom, M., Melzer, N., Schattling, B., Gob, E., Hicking, G., Arunachalam, P., Bittner, S., Ufer, F., Herrmann, A.M., Bernreuther, C., et al. (2014). Transient receptor potential melastatin subfamily member 2 cation channel regulates detrimental immune cell invasion in ischemic stroke. Stroke; a journal of cerebral 
circulation 45, 3395-3402.

Goldberg, M.P., and Choi, D.W. (1993). Combined oxygen and glucose deprivation in cortical cell culture: calcium-dependent and calcium-independent mechanisms of neuronal injury. The Journal of neuroscience : the official journal of the Society for Neuroscience 13, 3510-3524.

Granzotto, A., Canzoniero, L.M.T., and Sensi, S.L. (2020). A Neurotoxic Menage-a-trois: Glutamate, Calcium, and Zinc in the Excitotoxic Cascade. Frontiers in molecular neuroscience 13, 600089.

Hara, Y., Wakamori, M., Ishii, M., Maeno, E., Nishida, M., Yoshida, T., Yamada, H., Shimizu, S., Mori, E., Kudoh, J., et al. (2002). LTRPC2 Ca2+-permeable channel activated by changes in redox status confers susceptibility to cell death. Molecular cell 9, 163-173.

Hardingham, G. (2019). NMDA receptor C-terminal signaling in development, plasticity, and disease. F1000Research 8.

Hardingham, G.E., and Bading, H. (2010). Synaptic versus extrasynaptic NMDA receptor signalling: implications for neurodegenerative disorders. Nature reviews Neuroscience 11, 682-696.

Hardingham, G.E., Fukunaga, Y., and Bading, H. (2002). Extrasynaptic NMDARs oppose synaptic NMDARs by triggering CREB shut-off and cell death pathways. Nature neuroscience 5, 405-414.

Hecquet, C.M., Zhang, M., Mittal, M., Vogel, S.M., Di, A., Gao, X., Bonini, M.G., and Malik, A.B. (2014). Cooperative interaction of trp melastatin channel transient receptor potential (TRPM2) with its splice variant TRPM2 short variant is essential for endothelial cell apoptosis. Circulation research 114, 469-479.

Hirschberg, K., Miller, C.M., Ellenberg, J., Presley, J.F., Siggia, E.D., Phair, R.D., and Lippincott-Schwartz, J. (1998). Kinetic analysis of secretory protein traffic and characterization of golgi to plasma membrane transport intermediates in living cells. The Journal of cell biology 143, 1485-1503.

Horak, M., and Wenthold, R.J. (2009). Different roles of C-terminal cassettes in the trafficking of full-length NR1 subunits to the cell surface. The Journal of biological chemistry $284,9683-9691$.

Huang, Y., Roth, B., Lu, W., and Du, J. (2019). Ligand recognition and gating mechanism through three ligandbinding sites of human TRPM2 channel. eLife 8.

Huang, Y., Winkler, P.A., Sun, W., Lu, W., and Du, J. (2018). Architecture of the TRPM2 channel and its activation mechanism by ADP-ribose and calcium. Nature 562, 145-149.

Jia, J., Verma, S., Nakayama, S., Quillinan, N., Grafe, M.R., Hurn, P.D., and Herson, P.S. (2011). Sex differences in neuroprotection provided by inhibition of TRPM2 channels following experimental stroke. Journal of cerebral blood flow and metabolism : official journal of the International Society of Cerebral Blood Flow and Metabolism 31, 2160-2168.

Jiang, J., Li, M., and Yue, L. (2005). Potentiation of TRPM7 inward currents by protons. J Gen Physiol 126, 137150.

Kashio, M., Sokabe, T., Shintaku, K., Uematsu, T., Fukuta, N., Kobayashi, N., Mori, Y., and Tominaga, M. (2012). Redox signal-mediated sensitization of transient receptor potential melastatin 2 (TRPM2) to temperature affects macrophage functions. Proceedings of the National Academy of Sciences of the United States of America 109, 6745-6750.

Kashio, M., and Tominaga, M. (2017). The TRPM2 channel: A thermo-sensitive metabolic sensor. Channels 11, 426-433.

Keelan, J., Vergun, O., and Duchen, M.R. (1999). Excitotoxic mitochondrial depolarisation requires both calcium and nitric oxide in rat hippocampal neurons. The Journal of physiology 520 Pt 3, 797-813. 
Kuhn, F.J., Kuhn, C., Winking, M., Hoffmann, D.C., and Luckhoff, A. (2016). ADP-Ribose Activates the TRPM2 Channel from the Sea Anemone Nematostella vectensis Independently of the NUDT9H Domain. PloS one 11, e0158060.

Kuhn, F.J., and Luckhoff, A. (2004). Sites of the NUDT9-H domain critical for ADP-ribose activation of the cation channel TRPM2. The Journal of biological chemistry 279, 46431-46437.

Lan, J.Y., Skeberdis, V.A., Jover, T., Grooms, S.Y., Lin, Y., Araneda, R.C., Zheng, X., Bennett, M.V., and Zukin, R.S. (2001). Protein kinase $C$ modulates NMDA receptor trafficking and gating. Nature neuroscience 4, $382-$ 390.

Lemasters, J.J., Theruvath, T.P., Zhong, Z., and Nieminen, A.L. (2009). Mitochondrial calcium and the permeability transition in cell death. Biochimica et biophysica acta 1787, 1395-1401.

Lipton, P. (1999). Ischemic cell death in brain neurons. Physiol Rev 79, 1431-1568.

Liu, F., and McCullough, L.D. (2014). The middle cerebral artery occlusion model of transient focal cerebral ischemia. Methods in molecular biology 1135, 81-93.

Longa, E.Z., Weinstein, P.R., Carlson, S., and Cummins, R. (1989). Reversible middle cerebral artery occlusion without craniectomy in rats. Stroke; a journal of cerebral circulation 20, 84-91.

Mai, C., Mankoo, H., Wei, L., An, X., Li, C., Li, D., and Jiang, L.H. (2020). TRPM2 channel: A novel target for alleviating ischaemia-reperfusion, chronic cerebral hypo-perfusion and neonatal hypoxic-ischaemic brain damage. Journal of cellular and molecular medicine $24,4-12$.

Miller, B.A., Wang, J., Hirschler-Laszkiewicz, I., Gao, E., Song, J., Zhang, X.Q., Koch, W.J., Madesh, M., Mallilankaraman, K., Gu, T., et al. (2013). The second member of transient receptor potential-melastatin channel family protects hearts from ischemia-reperfusion injury. American journal of physiology Heart and circulatory physiology 304, H1010-1022.

Montell, C., Birnbaumer, L., and Flockerzi, V. (2002). The TRP channels, a remarkably functional family. Cell 108, 595-598.

Mortadza, S.S., Sim, J.A., Stacey, M., and Jiang, L.H. (2017). Signalling mechanisms mediating Zn(2+)-induced TRPM2 channel activation and cell death in microglial cells. Scientific reports 7, 45032.

Nguyen, P.V., Marin, L., and Atwood, H.L. (1997). Synaptic physiology and mitochondrial function in crayfish tonic and phasic motor neurons. Journal of neurophysiology 78, 281-294.

Nicolai, J., Burbassi, S., Rubin, J., and Meucci, O. (2010). CXCL12 inhibits expression of the NMDA receptor's NR2B subunit through a histone deacetylase-dependent pathway contributing to neuronal survival. Cell death \& disease 1 , e33.

Olah, M.E., Jackson, M.F., Li, H., Perez, Y., Sun, H.S., Kiyonaka, S., Mori, Y., Tymianski, M., and MacDonald, J.F. (2009). Ca2+-dependent induction of TRPM2 currents in hippocampal neurons. The Journal of physiology 587, 965-979.

Olney, J.W. (1969). Brain lesions, obesity, and other disturbances in mice treated with monosodium glutamate. Science 164, 719-721.

Perraud, A.L., Fleig, A., Dunn, C.A., Bagley, L.A., Launay, P., Schmitz, C., Stokes, A.J., Zhu, Q., Bessman, M.J., Penner, R., et al. (2001). ADP-ribose gating of the calcium-permeable LTRPC2 channel revealed by Nudix motif homology. Nature 411, 595-599.

Perraud, A.L., Schmitz, C., and Scharenberg, A.M. (2003). TRPM2 Ca2+ permeable cation channels: from gene to biological function. Cell calcium 33, 519-531. 
Petit-Pedrol, M., and Groc, L. (2021). Regulation of membrane NMDA receptors by dynamics and protein interactions. The Journal of cell biology 220.

Qiu, J., Tan, Y.W., Hagenston, A.M., Martel, M.A., Kneisel, N., Skehel, P.A., Wyllie, D.J., Bading, H., and Hardingham, G.E. (2013). Mitochondrial calcium uniporter Mcu controls excitotoxicity and is transcriptionally repressed by neuroprotective nuclear calcium signals. Nature communications 4, 2034.

Ren, M., Senatorov, V.V., Chen, R.W., and Chuang, D.M. (2003). Postinsult treatment with lithium reduces brain damage and facilitates neurological recovery in a rat ischemia/reperfusion model. Proceedings of the National Academy of Sciences of the United States of America 100, 6210-6215.

Sano, Y., Inamura, K., Miyake, A., Mochizuki, S., Yokoi, H., Matsushime, H., and Furuichi, K. (2001). Immunocyte Ca2+ influx system mediated by LTRPC2. Science 293, 1327-1330.

Sans, N., Prybylowski, K., Petralia, R.S., Chang, K., Wang, Y.X., Racca, C., Vicini, S., and Wenthold, R.J. (2003). NMDA receptor trafficking through an interaction between PDZ proteins and the exocyst complex. Nature cell biology 5, 520-530.

Schinder, A.F., Olson, E.C., Spitzer, N.C., and Montal, M. (1996). Mitochondrial dysfunction is a primary event in glutamate neurotoxicity. The Journal of neuroscience : the official journal of the Society for Neuroscience 16 , 6125-6133.

Scott, D.B., Blanpied, T.A., Swanson, G.T., Zhang, C., and Ehlers, M.D. (2001). An NMDA receptor ER retention signal regulated by phosphorylation and alternative splicing. The Journal of neuroscience : the official journal of the Society for Neuroscience 21, 3063-3072.

Sena, E., van der Worp, H.B., Howells, D., and Macleod, M. (2007). How can we improve the pre-clinical development of drugs for stroke? Trends in neurosciences 30, 433-439.

Shimizu, T., Macey, T.A., Quillinan, N., Klawitter, J., Perraud, A.L., Traystman, R.J., and Herson, P.S. (2013). Androgen and PARP-1 regulation of TRPM2 channels after ischemic injury. Journal of cerebral blood flow and metabolism : official journal of the International Society of Cerebral Blood Flow and Metabolism 33, 1549-1555.

Song, K., Wang, H., Kamm, G.B., Pohle, J., Reis, F.C., Heppenstall, P., Wende, H., and Siemens, J. (2016). The TRPM2 channel is a hypothalamic heat sensor that limits fever and can drive hypothermia. Science 353, 1393-1398.

Standley, S., Roche, K.W., McCallum, J., Sans, N., and Wenthold, R.J. (2000). PDZ domain suppression of an ER retention signal in NMDA receptor NR1 splice variants. Neuron 28, 887-898.

Starkus, J.G., Fleig, A., and Penner, R. (2010). The calcium-permeable non-selective cation channel TRPM2 is modulated by cellular acidification. The Journal of physiology 588, 1227-1240.

Takahashi, N., Kozai, D., Kobayashi, R., Ebert, M., and Mori, Y. (2011). Roles of TRPM2 in oxidative stress. Cell calcium 50, 279-287.

Tan, C.H., and McNaughton, P.A. (2016). The TRPM2 ion channel is required for sensitivity to warmth. Nature $536,460-463$.

Thompson, R.J., Zhou, N., and MacVicar, B.A. (2006). Ischemia opens neuronal gap junction hemichannels. Science 312, 924-927.

Tymianski, M. (2011). Emerging mechanisms of disrupted cellular signaling in brain ischemia. Nature neuroscience 14, 1369-1373.

Vergun, O., Keelan, J., Khodorov, B.I., and Duchen, M.R. (1999). Glutamate-induced mitochondrial depolarisation and perturbation of calcium homeostasis in cultured rat hippocampal neurones. The Journal of 
physiology 519 Pt 2, 451-466.

Vilar, B., Tan, C.H., and McNaughton, P.A. (2020). Heat detection by the TRPM2 ion channel. Nature 584, E5E12.

Virani, S.S., Alonso, A., Benjamin, E.J., Bittencourt, M.S., Callaway, C.W., Carson, A.P., Chamberlain, A.M., Chang, A.R., Cheng, S., Delling, F.N., et al. (2020). Heart Disease and Stroke Statistics-2020 Update: A Report From the American Heart Association. Circulation 141, e139-e596.

Wang, L., Fu, T.M., Zhou, Y., Xia, S., Greka, A., and Wu, H. (2018). Structures and gating mechanism of human TRPM2. Science 362.

Weilinger, N.L., Lohman, A.W., Rakai, B.D., Ma, E.M., Bialecki, J., Maslieieva, V., Rilea, T., Bandet, M.V., Ikuta, N.T., Scott, L., et al. (2016). Metabotropic NMDA receptor signaling couples Src family kinases to pannexin-1 during excitotoxicity. Nature neuroscience 19, 432-442.

White, R.J., and Reynolds, I.J. (1996). Mitochondrial depolarization in glutamate-stimulated neurons: an early signal specific to excitotoxin exposure. The Journal of neuroscience : the official journal of the Society for Neuroscience 16, 5688-5697.

Wu, L.J., Wu, G., Akhavan Sharif, M.R., Baker, A., Jia, Y., Fahey, F.H., Luo, H.R., Feener, E.P., and Clapham, D.E. (2012). The voltage-gated proton channel $\mathrm{H} v 1$ enhances brain damage from ischemic stroke. Nature neuroscience $15,565-573$.

Wu, Q.J., and Tymianski, M. (2018). Targeting NMDA receptors in stroke: new hope in neuroprotection. Mol Brain 11, 15.

Xie, J., Bi, Y., Zhang, H., Dong, S., Teng, L., Lee, R.J., and Yang, Z. (2020). Cell-Penetrating Peptides in Diagnosis and Treatment of Human Diseases: From Preclinical Research to Clinical Application. Frontiers in pharmacology 11, 697 .

Xie, Y.-F., Belrose, J.C., Lei, G., Tymianski, M., Mori, Y., MacDonald, J.F., and Jackson, M.F. (2011). Dependence of NMDA/GSK-3 $\beta$ Mediated Metaplasticity on TRPM2 Channels at Hippocampal CA3-CA1 Synapses. Molecular Brain 4, 44.

Xiong, Z.G., Raouf, R., Lu, W.Y., Wang, L.Y., Orser, B.A., Dudek, E.M., Browning, M.D., and MacDonald, J.F. (1998). Regulation of N-methyl-D-aspartate receptor function by constitutively active protein kinase C. Molecular pharmacology 54, 1055-1063.

Yamamoto, S., Shimizu, S., Kiyonaka, S., Takahashi, N., Wajima, T., Hara, Y., Negoro, T., Hiroi, T., Kiuchi, Y., Okada, T., et al. (2008). TRPM2-mediated Ca2+influx induces chemokine production in monocytes that aggravates inflammatory neutrophil infiltration. Nature medicine 14, 738-747.

Yan, J., Bengtson, C.P., Buchthal, B., Hagenston, A.M., and Bading, H. (2020). Coupling of NMDA receptors and TRPM4 guides discovery of unconventional neuroprotectants. Science 370.

Yan, J.Z., Xu, Z., Ren, S.Q., Hu, B., Yao, W., Wang, S.H., Liu, S.Y., and Lu, W. (2011). Protein kinase C promotes $\mathrm{N}$-methyl-D-aspartate (NMDA) receptor trafficking by indirectly triggering calcium/calmodulindependent protein kinase II (CaMKII) autophosphorylation. The Journal of biological chemistry 286, 2518725200.

Yang, W., Zou, J., Xia, R., Vaal, M.L., Seymour, V.A., Luo, J., Beech, D.J., and Jiang, L.H. (2010). Statedependent inhibition of TRPM2 channel by acidic pH. The Journal of biological chemistry 285, 30411-30418.

Yu, P., Xue, X., Zhang, J., Hu, X., Wu, Y., Jiang, L.H., Jin, H., Luo, J., Zhang, L., Liu, Z., et al. (2017). Identification of the ADPR binding pocket in the NUDT9 homology domain of TRPM2. The Journal of general 
physiology.

Zeng, H., Guo, M., Martins-Taylor, K., Wang, X., Zhang, Z., Park, J.W., Zhan, S., Kronenberg, M.S., Lichtler, A., Liu, H.X., et al. (2010). Specification of region-specific neurons including forebrain glutamatergic neurons from human induced pluripotent stem cells. PloS one 5, e11853.

Zhang, C., Brown, M.Q., van de Ven, W., Zhang, Z.M., Wu, B., Young, M.C., Synek, L., Borchardt, D., Harrison, R., Pan, S., et al. (2016). Endosidin2 targets conserved exocyst complex subunit EXO70 to inhibit exocytosis. Proceedings of the National Academy of Sciences of the United States of America 113, E41-50.

Zhang, D., Jin, W., Liu, H., Liang, T., Peng, Y., Zhang, J., and Zhang, Y. (2020). ENT1 inhibition attenuates apoptosis by activation of $\mathrm{CAMP} / \mathrm{pCREB} / \mathrm{Bcl} 2$ pathway after MCAO in rats. Experimental neurology 331 , 113362.

Zheng, X., Zhang, L., Wang, A.P., Bennett, M.V., and Zukin, R.S. (1999). Protein kinase C potentiation of Nmethyl-D-aspartate receptor activity is not mediated by phosphorylation of $\mathrm{N}$-methyl-D-aspartate receptor subunits. Proceedings of the National Academy of Sciences of the United States of America 96, 15262-15267. 
bioRxiv preprint doi: https://doi.org/10.1101/2021.07.29.454247; this version posted July 30, 2021. The copyright holder for this preprint (which was not certified by peer review) is the author/funder. All rights reserved. No reuse allowed without permission.

\section{STAR * METHODS}

Table 1

KEY RESOURCES TABLE

\begin{tabular}{|c|c|c|}
\hline REAGENT or RESOURCE & SOURCE & IDENTIFIER \\
\hline \multicolumn{3}{|l|}{ Antibodies } \\
\hline Rabbit polyclonal antibodies to TRPM2 & Novus & NB110-81601 \\
\hline Rabbit polyclonal antibodies to TRPM2 $\mathrm{N}$ terminal part & Abmart & $634-1-1-R 1$ \\
\hline Rabbit polyclonal antibodies to TRPM2 C terminal part & Abmart & 634-2-1-R1 \\
\hline Rabbit polyclonal antibodies to GluN1 & Cell Signaling Technology & $5704 S$ \\
\hline Rabbit polyclonal antibodies to GluN2A & Cell Signaling Technology & $4205 S$ \\
\hline Rabbit polyclonal antibodies to GluN2B & Cell Signaling Technology & 4207S \\
\hline Mouse monoclonal antibodies to flag & Sigma-Aldrich & F3165 \\
\hline Rabbit polyclonal antibodies to phosphor-CREB(Ser133) & Cell Signaling Technology & $9198 S$ \\
\hline Rabbit polyclonal antibodies to p44/42 MAPK (ERK1/2) & Cell Signaling Technology & $9102 S$ \\
\hline Rabbit polyclonal antibodies to phospho-p44/42 MAPK (ERK1/2) (Tyr202/204) & Cell Signaling Technology & $4377 \mathrm{~T}$ \\
\hline Rabbit polyclonal antibodies to PKC-Y & Cell Signaling Technology & $59090 \mathrm{~S}$ \\
\hline Rabbit polyclonal antibodies to Pan-cadherin & Cell Signaling Technology & 4068S \\
\hline Rabbit polyclonal antibodies to GAPDH & Cell Signaling Technology & $2118 S$ \\
\hline Rabbit polyclonal antibodies to $\beta$-tubulin & Cell Signaling Technology & $4820 S$ \\
\hline Rabbit polyclonal antibodies to NeuN & Abcam & ab187477 \\
\hline Goat anti-rabbit IgG-FITC & Santa Cruz Biotechnology & sc-2012 \\
\hline Goat anti-mouse IgG-rhodamine & Thermal Fisher Scientific & 31160 \\
\hline \multicolumn{3}{|l|}{ Chemicals and peptides } \\
\hline 2,3,5-Triphenyltetrazolium chloride & Sigma-Aldrich & T-8877 \\
\hline N-Methyl-D-aspartic acid & Tocris & 0114 \\
\hline Glutamate & Sigma-Aldrich & 49621 \\
\hline Glycine & Sigma-Aldrich & 50046 \\
\hline Bicuculine & $\mathrm{TCl}$ & $\mathrm{B} 1890$ \\
\hline 4-Aminopyridine & Sigma-Aldrich & A-0152 \\
\hline MK-801 & Sigma-Aldrich & M107 \\
\hline Phorbol-12-myristate-13-acetate & Sigma-Aldrich & 524400 \\
\hline $30 \%$ Hydrogen Peroxide & Thermal Fisher Scientific & 200745 \\
\hline NP40 & Thermal Fisher Scientific & 28324 \\
\hline Triton $^{\mathrm{TM}} \mathrm{X}-100$ & Sigma-Aldrich & T-9284 \\
\hline Bovine Serum Albumin & Sigma-Aldrich & $9048-46-8$ \\
\hline Goat Serum & Thermal Fisher Scientific & $16210-064$ \\
\hline Rhodamine-123 & Thermal Fisher Scientific & $\mathrm{R} 302$ \\
\hline Fura-2 AM & Thermal Fisher Scientific & $\mathrm{F} 1221$ \\
\hline lonomycin & Sigma-Aldrich & 10634 \\
\hline Pluronic $^{\mathrm{TM}} \mathrm{F}-127$ & Thermal Fisher Scientific & P3000MP \\
\hline Proteinase inhibitors & Sigma-Aldrich & 539131-10VL \\
\hline Phosphatase inhibitors & Thermal Fisher Scientific & 78428 \\
\hline Laemmli Sample Buffer & BIO-RAD & 1610737 \\
\hline Protein A/G PLUS-Agarose & Santa Cruz Biotechnology & sc-2003 \\
\hline TAT-SC & Genescript & $\mathrm{N} / \mathrm{A}$ \\
\hline TAT-EE ${ }_{3}$ & Genescript & $\mathrm{N} / \mathrm{A}$ \\
\hline
\end{tabular}


bioRxiv preprint doi: https://doi.org/10.1101/2021.07.29.454247; this version posted July 30, 2021. The copyright holder for this preprint (which was not certified by peer review) is the author/funder. All rights reserved. No reuse allowed without permission.

\begin{tabular}{|c|c|c|}
\hline \multicolumn{3}{|l|}{ Continued } \\
\hline REAGENT or RESOURCE & SOURCE & IDENTIFIER \\
\hline \multicolumn{3}{|l|}{ Chemicals and peptides } \\
\hline \multicolumn{3}{|c|}{ All chemicals for making artificial cerebrospinal fluid (aCSF; see below) and recording solution (see below) were purchased from Sigma-Aldrich. } \\
\hline \multicolumn{3}{|l|}{ Plasmids and enzymes } \\
\hline GluN1a & Addgene & 17928 \\
\hline GluN2A & Addgene & 17924 \\
\hline GluN2B & Addgene & 17925 \\
\hline PKC-Y & Addgene & 112266 \\
\hline PKC-Y-DN & Addgene & 21239 \\
\hline pcDNA4/TO-FLAG-hTRPM2 & $\begin{array}{l}\text { Dr. Sharenberg AM (Unive } \\
\text { et al., 2003). }\end{array}$ & ggton, Seattle)(Perraud \\
\hline Xbal & BioLabs & R0145S \\
\hline Xhol & BioLabs & R0146S \\
\hline BamHI & BioLabs & R3136S \\
\hline Dpnl & BioLabs & R0176S \\
\hline EcoRI & BioLabs & R3101S \\
\hline T4 DNA ligase & Thermal Fisher Scientific & 2148085 \\
\hline PfuUltra HF & Agilent & $600380-51$ \\
\hline Q5® High-Fidelity DNA Polymerase & BioLabs & M0491S \\
\hline \multicolumn{3}{|l|}{ Cell culture } \\
\hline Dulbecco's Modified Eagle's medium & Thermal Fisher Scientific & $12100-038$ \\
\hline Bovine Calf Serum & HyClone & $\mathrm{SH} 30541.03$ \\
\hline Penicillin/streptomycin & Thermal Fisher Scientific & 15140-122 \\
\hline $2.5 \%$ trypsin & Thermal Fisher Scientific & 15090-046 \\
\hline Neurobasal® Medium & Thermal Fisher Scientific & 21103-049 \\
\hline B27® supplement & Thermal Fisher Scientific & $17504-044$ \\
\hline Horse serum & Thermal Fisher Scientific & 16050114 \\
\hline L-glutamine & Thermal Fisher Scientific & $25030-081$ \\
\hline Cytosine arabinoside & Thermal Fisher Scientific & C1768 \\
\hline Poly-L-lysine & Sigma-Aldrich & P4707 \\
\hline \multicolumn{3}{|l|}{ Critical commercial assays } \\
\hline Lipofectamine® 3000 Transfection Kit & Thermal Fisher Scientific & 2232162 \\
\hline Pierce $^{\mathrm{TM}}$ Rapid Gold BCA Protein Assay Kit & Thermal Fisher Scientific & A53225 \\
\hline Pierce $\circledast$ Cell Surface Protein Isolation Kit & Thermal Fisher Scientific & 89881 \\
\hline ProteoExtract ${ }^{\mathrm{TM}}$ Native Membrane Protein Extraction Kit & Calbiochem & 444810 \\
\hline Fisher Healthcare $^{\mathrm{TM}}$ Tissue-Plus $^{\mathrm{TM}}$ O.C.T. Compound & Thermal Fisher Scientific & $23-730-571$ \\
\hline ProLong ${ }^{\mathrm{TM}}$ Gold Antifade Mountant & Thermal Fisher Scientific & P10144 \\
\hline QIAprep® Spin Miniprep Kit & QIAGEN & 27106 \\
\hline QIAGEN® Plasmid Maxi Kit & QIAGEN & 12163 \\
\hline Qiaquick $\circledast$ PCR Purification Kit & QIAGEN & 28104 \\
\hline In Situ Cell Death Detection Kit & Millipore Sigma & 11684795910 \\
\hline
\end{tabular}




\begin{tabular}{|c|c|c|}
\hline \multicolumn{3}{|l|}{ Continued } \\
\hline REAGENT or RESOURCE & SOURCE & IDENTIFIER \\
\hline \multicolumn{3}{|l|}{ Oligonucleotides } \\
\hline $\begin{array}{l}\text { Primers for TRPM2 subcloning and mutagenesis, see Table } \\
\text { S1 }\end{array}$ & This study & N/A \\
\hline $\begin{array}{l}\text { Primers for GluN2a and GluN2b subcloning and } \\
\text { mutagenesis, see Table } \mathrm{S} 1\end{array}$ & This study & N/A \\
\hline \multicolumn{3}{|l|}{ Software } \\
\hline GraphPad Prism 6.0 & GraphPad Software & N/A \\
\hline Biorender & Biorender & N/A \\
\hline Adobe Illustrator & Adobe & N/A \\
\hline NIS Elements AR4 & Nikon & N/A \\
\hline pClamp 9.2 & Molecular Devices & N/A \\
\hline
\end{tabular}

\section{CONTACT FOR REAGENT AND RESOURCE SHARING}

Further information and resources and reagents should be directed to and will be fulfilled by the lead contact, Dr. Lixia Yue (lyue@uchc.edu)

\section{EXPERIMENTAL MODEL AND SUBJECT DETAILS}

\section{Animal Models}

\section{Animal Care}

All the experimental mice bred and hosted in the animal facility building of University of Connecticut School of Medicine (UCONN Health) were fed with standard chow diet and water ad libitum. Standard housing conditions were maintained at a controlled temperature with a 12-h light/dark cycle. All experimental procedures and protocols were approved by the Institutional Animal Care and Use Committee (IACUC) of University of Connecticut School of Medicine (animal protocol: AP-200135-0723), and were conducted in accordance with the U.S. National Institutes of Health Guidelines for the Care and Use of Laboratory Animals.

\section{Knockout of TRPM2 (TRPM2-KO)}

The global TRPM2 knockout (TRPM2-KO, or gM2KO) mice were generated by Dr. Yasuo Mori's lab at Kyoto 
University Japan. The deletion of Trpm2 was developed in C57B6J mouse by replacing the third exon (S5-S6 linker in the pore domain) with a neomycin coding region. The knockout mice exhibited no differences in behavior or impairment in breeding, compared to wild type (WT) C57BJ6 mice (Yamamoto et al., 2008). TRPM2-KO mice were back-crossed to C57BL/6 mice for $\geq 10$ generations before being used for experiments.

The neuron specific knockout of TRPM2 (nM2KO) was generated by breeding TRPM2 ${ }^{\mathrm{fl} / \mathrm{fl}}$ mice with Nestin-Cre ((B6.Cg-Tg)Nes-cre)1kln/J: 003771; JAX laboratory). TRPM2 $2^{\mathrm{fl} / \mathrm{fl}}$ mice were generated by Dr. Barbara Miller (Miller et al., 2013) (Penn State University, Pennsylvania). The exons 21 and 22 encoding transmembrane domain 5 and 6 and pore loop were flanked by loxp recombination sites and will be deleted by Cre recombinase (Miller et al., 2013). The mice were backcrossed with C57BL/6 mice for $\geq 10$ generations before being used for experiments. The TRPM2 ${ }^{\text {floxfllox }}\left(\right.$ TRPM2 $\left.^{f|f| f l}\right)$ with $\mathrm{Cre}^{+}$mice and TRPM2 ${ }^{\text {fl/fl }}$ with $\mathrm{Cre}^{-}$mice from the same litters were paired for experiments throughout the manuscript.

The inducible global knockout was also generated by using TRPM2 $2^{f / f l}$ mice breeding with global Cre, Rosa26CreERT2 (B6.129-Gt(ROSA)26Sortm1(cre/ERT2)Tyj/J: 008463; JAX laboratory). Knockout was induced by Tamoxifen treatment and confirmed by genotyping. The mice were backcrossed with C57BL/6 mice for $\geq 10$ generations before being used for experiments.

\section{Middle cerebral artery occlusion (MCAO)}

Eight- to nine-week-old male mice $(\sim 25 \mathrm{~g})$ were subjected to right middle cerebral artery occlusion (MCAO) for 120 min followed by 24 hours of reperfusion. The genotype information was blinded to the surgeon who conduct the surgeries. MCAO surgery was performed as previously described (Liu and McCullough, 2014; Wu et al., 2012). In brief, mice were anesthetized with $2 \%$ isoflurane (vol/vol) in $100 \%$ oxygen and the anesthesia was maintained with $1.5 \%$ isoflurane during surgery through nose cone (Harvard Apparatus). The unilateral right middle cerebral artery (MCA) occlusion was carried out by advancing a silicone-coated 6-0 monofilament (Doccol Corporation, 
Sharon, MA) 10 to $11 \mathrm{~mm}$ from internal carotid artery bifurcation via an external carotid artery incision (Chiang et al., 2011). Mouse body temperature was monitored by a rectal temperature probe and maintained at $\sim 37^{\circ} \mathrm{C}$ with an automatic temperature-regulating heating pad connected to animal temperature controller (TCA T-2DF, Physitemp). Cerebral blood flow was monitored after occlusion as well as after reperfusion. The bregma was exposed and the skull bone countersunk at two $3 \times 3-\mathrm{mm}$ areas over both MCA supply territories for bilateral monitoring of local cortical blood flow. Successful occlusion was confirmed by $85 \%$ reduction of cerebral blood flow monitored by Laser Doppler Flowmetry (LDF) with laser Doppler blood FlowMeter (Moor-VMS-LDF1, Moor Instrument, Dever, UK). Sham control mice underwent the same procedure but without insertion of filament to occlude the MCA.

\section{Neurological deficit score evaluation}

After 24 hours of MCAO or sham procedures, neurological deficit was scored based on previously reported criteria (Longa et al., 1989). In brief, score 0 is the minimum (best) score representing no neurological deficit; score 1 represents failure to extend left paw; Score 2 represents circling to the left; score 3 represents falling to the left; score 4 represents inability of spontaneously walking and decreased level of consciousness; and score 5 represents death due to brain ischemia. The observer to score the neurological deficit was an experienced observer and blinded by the group assignment and genotype information. If the animal score was 0 or 5 , it was removed from the study.

\section{Infarct volume assessment by Triphenyl Tetrazolium chloride (TTC) staining}

Tetrazolium chloride (Sigma-Aldrich, T-8877) was dissolved in PBS at a concentration of $20 \mathrm{mg} / \mathrm{ml} 30 \mathrm{~min}$ prior to use. Post-stroke mice were euthanized and brains were frozen at $-80^{\circ} \mathrm{C}$ for $5 \mathrm{~min}$, cut into coronary slices at a 
thickness of $1 \mathrm{~mm}$. Brain slices were stained with 2\% TTC (vol/vol) for $20 \mathrm{~min}$, and then washed using PBS for 3 times, and fixed in 10\% Neutral buffered formalin for later scanning. TTC labels non-injured tissue, leaving the infarct area white. The stained slices were scanned for data analysis using ImageJ software. The infarct volume was calculated and presented as a percentage of total brain volume (Ren et al., 2003).

\section{Antibodies, chemicals and reagents}

Rabbit polyclonal antibodies to TRPM2 (Novus, NB110-81601, 1:500 in 5\% BSA for WB, 1:50 in protein extraction for IP); Rabbit polyclonal antibodies to GluN1 (Cell Signaling Technology, 5704S, 1:1000 in 5\% BSA for WB, 1:50 in protein extraction for IP); Rabbit polyclonal antibodies to GluN2A (Cell Signaling Technology, 4205S, 1:1000 in 5\% BSA for WB, 1:50 in protein extraction for IP). Rabbit polyclonal antibodies to GluN2B (Cell Signaling Technology, 4207S, 1:1000 in 5\% BSA for WB, 1:50 in protein extraction for IP); Mouse polyclonal antibodies to flag (Sigma-Aldrich, F3165, 1:5000 in 5\% BSA for WB): Rabbit polyclonal antibodies to GFP (Cell Signaling Technology, 2956S, 1:2000 in 5\% BSA for WB); Rabbit polyclonal antibodies to CREB (Cell Signaling Technology, 4820S, 1:2000 in 5\% BSA for WB); Rabbit polyclonal antibodies to phosphor-CREB(Ser133) (Cell Signaling Technology, 9198S, 1:2000 in 5\% BSA for WB); Rabbit polyclonal antibodies to p44/42 MAPK (ERK1/2) (Cell Signaling Technology, 9102S, 1:2000 in 5\% BSA for WB); Rabbit polyclonal antibodies to phospho-p44/42 MAPK (ERK1/2) (Tyr202/204) (Cell Signaling Technology, 4377T, 1:2000 in 5\% BSA for WB); Rabbit polyclonal antibodies to PKC-y (Cell Signaling Technology, 59090S, 1:5000 in 5\% BSA for WB); Rabbit polyclonal antibodies to Pan-cadherin (Cell Signaling Technology, 4068S, 1:5000 in 5\% BSA for WB); Rabbit polyclonal antibodies to GAPDH (Cell Signaling Technology, 2118S, 1:5000 in 5\% BSA for WB); Rabbit polyclonal antibodies to $\beta$-tubulin

(Cell Signaling Technology, 4820S, 1:5000 in 5\% BSA for WB); Mouse monoclonal antibodies to Caspase-3 (Santa Cruz Biotechnology, sc-7272, 1:1000 in 5\% BSA for WB, 1:50 in 5\% BSA and 15\% goat serum for immunofluorescence staining); Rabbit polyclonal antibodies to NeuN (Abcam, ab187477, 1:50 in 5\% BSA and 15\% 
goat serum for immunofluorescence staining); Goat anti-rabbit IgG-FITC (Santa Cruz Biotechnology, sc-2012, 1:1000 in 5\% BSA and 15\% goat serum for immunofluorescence staining); Goat anti-mouse IgG-rhodamine (Thermal Fisher Scientific, 31660, 1:1000 in 5\% BSA and 15\% goat serum for immunofluorescence staining); Prolong® Gold antifade reagent with DAPI (Life technologies, P36935), HRP-linked anti-rabbit lgG (Cell Signaling Technology, 7074S, 1:10000 in 5\% BSA for WB); HRP-linked anti-mouse-lgG (Cell Signaling Technology, 7076S, 1:10000 in 5\% BSA for WB); Tetrazolium chloride (Sigma-Aldrich, T-8877); NMDA (Tocris, 0114); Glutamate (Sigma-Aldrich, 49621); Glycine(Sigma-Aldrich, 50046); Bicuculine (TCI, B1890); 4-AP (Sigma-Aldrich, A-0152); MK-801 (Sigma-Aldrich, M107); PMA (Sigma-Aldrich, 524400); 4- $\alpha-P M A$ (Sigma-Aldrich, P128); $\mathrm{H}_{2} \mathrm{O}_{2}$ (Thermal Fisher Scientific, 200745); NP40 (Thermal Fisher Scientific, 28324), Triton ${ }^{\mathrm{TM}}$ X-100 (T-9284), Bovine Serum Albumin (Sigma-Aldrich, 9048-46-8), Goat Serum (Thermal Fisher Scientific, 16210-064). All chemicals for making artificial cerebrospinal fluid (aCSF; see below) and recording solution (see below) were purchased from Sigma-Aldrich.

Membrane permeable peptide TAT-EE for $_{3}$ disrupting TRPM2 and NMDARs coupling and scramble control

\section{TAT-SC peptides}

TAT-SC (sequence: YGRKKRRQRRR VILLKDHTLEYPVF) and TAT-EE 3 (sequence: YGRKKRRQRRR EEDTDSSEEMLALAEE) were ordered from GenScript Biotech, and dissolved in PBS to make a stock concentration at $10 \mathrm{mM}$. HEK-293T cells or isolated neurons were treated with TAT-SC or TAT-EE 3 at a concentration of $10 \mu \mathrm{M}$ for at least $8 \mathrm{~h}$ prior to use. Mice were injected with TAT-SC or TAT-EE 3 intraperitoneally (ip) at a dose of $100 \mathrm{nmol} / \mathrm{kg}$.

\section{Plasmids and enzymes}

GluN1a (Addgene, 17928), GluN2A (Addgene, 17924), GluN2B (Addgene, 17925), PKC-y (Addgene, 112266), 
PKC-Y-DN (Addgene, 21239). The pcDNA4/TO-FLAG-hTRPM2 construct was a kind gift from Dr. Sharenberg AM (University of Washington, Seattle)(Perraud et al., 2003).

Xbal (BioLabs, R0145S), BamHI (BioLabs, R3136S), Xhol (BioLabs, R0146S), Dpnl (BioLabs, R0176S), EcoRI (BioLabs, R3101S) and T4 DNA ligase (Thermal Fisher Scientific, 2148085), PfuUltra HF (Agilent, 600380-51), and Q5® High-Fidelity DNA Polymerase (Biolabs, M0491S) were used to generating different deletion or mutation constructs.

\section{Subcloning}

For TRPM2, subcloning of $\mathrm{N}$ terminus (1-727) was achieved by introducing a stop codon (A2282T) by PCR using PfuUltra HF. C terminal of TRPM2 was amplified by PCR using Q5® High-Fidelity DNA Polymerase, cut by EcoRI and Xbal, and inserted into EGFP-C3 vector. To look for the binding part in N terminus of TRPM2, a series of stop codons were introduced by PCR using PfuUltra HF (C1831A, C1994T, G2138T, A2090T). EE3 motif was deleted by PCR using Q5® High-Fidelity DNA Polymerase. EE was mutated to QQ by PCR using PfuUltra HF (G2093C, G2096C; G2117C, G2120C; G2138C, G2141C). For GluN2A and GluN2B, C terminal was amplified by PCR using Q5® High-Fidelity DNA Polymerase, cut by EcoRI and Xbal, and inserted into EGFP-C3 vector. Deletion of C terminus of GluN2A and GluN2B was achieved by introducing a stop codon by PCR using PfuUltra HF (G4371T for GluN2B and G4518T for GluN2B). The information of all the primers is listed in the Table S1.

\section{Cell culture and transfection}

HEK293T cells were cultured in Dulbecco's Modified Eagle's medium (DMEM) (Thermal Fisher Scientific, 12100038) supplemented with 10\% BGS (HyClone, SH30541.03) and 0.5\% penicillin/streptomycin (Thermal Fisher Scientific, $15140-122)$ at $37^{\circ} \mathrm{C}$ and $5 \% \mathrm{CO} 2$. $8 \mathrm{~h}$ prior to transfection, culture medium was replaced with DMEM 
supplemented only with $2.5 \%$ BGS. Cells were transfected when at a confluence about $80-90 \%$ using Lipofectamine® 3000 Transfection Kit (Thermal Fisher Scientific, 2232162) based on instruction.

\section{Neuron isolation and culture}

Mice pups at P0 were euthanized based on animal protocol. Whole brain was dissected out immediately and immersed in ice-cold Hank's Balanced Salt Solution (HBSS). Meninges were removed thoroughly, and tissue of different brain areas was taken based on purposes. Brain tissue was cut into small pieces and digested with $0.25 \%$ trypsin (Thermal Fisher Scientific, 15090-046) in HBSS at $37^{\circ} \mathrm{C}$ for 20 min. Digestion solution was quickly removed, and tissue pellets are washed with Neurobasal® Medium (Thermal Fisher Scientific, 21103-049) for 3 times. Cells were resuspended with appropriate amount of Neurobasal® Medium supplemented with $2 \%$ B27® supplement (Thermal Fisher Scientific, 17504-044), 3\% horse serum (Thermal Fisher Scientific, 16050114), 0.25\% L-glutamine (Thermal Fisher Scientific, 25030-081) and 1\% penicillin/streptomycin (Thermal Fisher Scientific, 15140-122). Isolated cells were counted and plated on coverslips pre-coated with poly-L-lysine (Sigma-Aldrich, P4707) at a density about $500 \times 10^{3}$ cells $/ \mathrm{cm}^{2}$ for $\mathrm{OGD}$ and $\mathrm{H}_{2} \mathrm{O}_{2}$ treatment, and $100 \times 10^{3} \mathrm{cells} / \mathrm{cm}^{2}$ for current recording. Cytosine arabinoside (Sigma-Aldrich, C1768) was added to maintain a concentration at $1 \mu \mathrm{M}$ to inhibit the proliferation of non-neuronal cells. $24 \mathrm{~h}$ after plating, culture medium was changed to Neurobasal® Medium supplemented with $2 \%$ B27® supplement, $0.25 \%$ L-glutamine and 1\% penicillin/streptomycin. The concentration of Cytosine arabinoside (araC) was increased to $2 \mu \mathrm{M}$. Medium was changed every 3 days. OGD and $\mathrm{H}_{2} \mathrm{O}_{2}$ treatment was conducted at $7^{\text {th }}$ day of culture, and current recording was conducted at $7^{\text {th }}, 10^{\text {th }}$ and $14^{\text {th }}$ day of culture.

\section{Oxygen-glucose deprivation}

Oxygen-glucose deprivation (OGD) was achieved by replace the glucose in aCSF with sucrose, and $95 \% \mathrm{~N}_{2}$ and 
$5 \% \mathrm{CO}_{2}$ was used to equilibrate sucrose-aCSF to displace oxygen. This condition typically yielded a pO2 of $<5$ $\mathrm{mm} \mathrm{Hg}$ in the imaging chamber (Thompson et al., 2006). At least $10 \mathrm{~min}$ was allowed for neurons to adapt to the change from culture medium to aCSF before OGD was applied.

\section{Real-time monitoring of mitochondrial function}

Mitochondria function was evaluated using Rhodamine-123 dequenching as previously reported. Rhodamine123 (Rh123,Thermal Fisher Scientific, R302) was dissolved in DMSO to make a stock concentration at $10 \mathrm{mg} / \mathrm{ml}$. Pre-warmed Neurobasal ${ }^{\circledR}$ Medium was used to dilute Rhodamine-123 to $5 \mu \mathrm{g} / \mathrm{ml}$ as working concentration. Culture medium was removed and cultured neurons on the $25 \mathrm{~mm}$ coverslip were washed using prewarmed PBS for 3 times, then $2 \mathrm{ml}$ of Rh123 working solution was added. Cells were incubated with Rh123 at $37^{\circ} \mathrm{C}$ for 5 min. Then Rh123 working solution was replaced with culture medium. At least 10 min were allowed to achieve Rh123 equilibration after the transition of culture medium to aCSF before experiments.

Fluorescence intensities at $509 \mathrm{~nm}$ with excitation at $488 \mathrm{~nm}$ was collected every $15 \mathrm{~s}$ for 30 min using CoolSNAP HQ2 (Photometrics) and data were analyzed using NIS-Elements (Nikon).

\section{Ratio calcium imaging experiments}

Changes of intracellular $\mathrm{Ca}^{2+}$ was measured using ratio $\mathrm{Ca}^{2+}$ imaging as we described previously (Du et al., 2010). In brief, Fura-2 AM (Thermal Fisher Scientific, F1221) was dissolved in DMSO to make a stock concentration at $1 \mathrm{mM}$. Pre-warmed Neurobasal® Medium (Thermal Fisher Scientific, 21103-049) was used to dilute Fura-2 AM to a working concentration at $2.5 \mu \mathrm{M}$, and $0.02 \%$ Pluronic $^{\mathrm{TM}} \mathrm{F}-127$ (Thermal Fisher Scientific, P3000MP) was added to facilitate loading of Fura-2 AM. Cells plated on $25 \mathrm{~mm}$ glass coverslips were washed using pre-warmed PBS for 3 times, and then incubated with $2 \mathrm{ml}$ of Fura-2 AM working solution for $30 \sim 45 \mathrm{~min}$ at $37^{\circ} \mathrm{C}$. Nonincorporated dye was washed away using HEPES-buffered Saline Solution (HBSS) containing (in mM): 20 
HEPES, 10 glucose, $1.2 \mathrm{MgCl}_{2}, 1.2 \mathrm{KH}_{2} \mathrm{PO}_{4}, 4.7 \mathrm{KCl}, 140 \mathrm{NaCl}, 1.3 \mathrm{Ca}^{2+}(\mathrm{pH} 7.4)$.

$\mathrm{Ca}^{2+}$ influx was measured by perfusing the cells with Tyrode's solution for transfected HEK293T cells or aCSF for neurons under different conditions. Ionomycin (Iono) at $1 \mu \mathrm{M}$ was applied at the end of the experiment as an internal control. Fluorescence intensities at $510 \mathrm{~nm}$ with $340 \mathrm{~nm}$ and $380 \mathrm{~nm}$ excitation were collected at a rate of $1 \mathrm{~Hz}$ using CoolSNAP HQ2 (Photometrics) and data were analyzed using NIS-Elements (Nikon). The 340:380 $\mathrm{nm}$ ratio in the presence of different treatments was normalized to the maximal $\mathrm{Ca}^{2+}$ signal elicited by $1 \mu \mathrm{M}$ Ionomycin (lono) as we previously reported (Du et al., 2010).

\section{Co-immunoprecipitation}

NP-40 lysis buffer (10\% NP40, $150 \mathrm{mM} \mathrm{NaCl}, 1 \mathrm{mM}$ EDTA, $50 \mathrm{mM}$ Tris, $\mathrm{pH}=8.0$ ) containing proteinase inhibitors (Sigma-Aldrich, 539131-10VL) and phosphatase inhibitors (Thermal Fisher Scientific, 78428) was used to lyse both cultured cells and frozen brain tissue. For transfected cells, proteins were extracted 36 hours after transfection. Cell and tissue lysate were lysed by ultrasound using an ultrasonic cleaner (Thermal Fisher Scientific) filled with ice-cold water for $30 \mathrm{~min}$. After incubated on ice for $1 \mathrm{~h}$, lysate was centrifuged at $13000 \mathrm{~g}$ for $30 \mathrm{~min}$ and supernatant was collected. Protein concentration was measured using Pierce ${ }^{\mathrm{TM}}$ Rapid Gold BCA Protein Assay Kit (Thermal Fisher Scientific, A53225). $300 \mu \mathrm{g}$ of protein was taken and diluted using NP-40 lysis buffer to make a total volume of $500 \mu \mathrm{l}$. Unused protein was allocated and frozen at $-80^{\circ} \mathrm{C}$ for future use. Appropriate amount of antibody was added based on instruction. After protein-antibody mixture was incubated on ice for $2 \mathrm{~h}$, $25 \mu \mathrm{l}$ of pre-washed Protein A/G PLUS-Agarose (Santa Cruz Biotechnology, sc-2003) was added, and the whole mixture was incubated at $4{ }^{\circ} \mathrm{C}$ for overnight. Then the mixture was centrifuged at $2500 \mathrm{~g}$ for $1 \mathrm{~min}$ to get agarose beads. Agarose beads was washed using NP-40 lysis buffer for 7 times, mixed with same amount of $2 x$ Laemmli Sample Buffer (BIO-RAD, 1610737), and boiled at $95^{\circ} \mathrm{C}$ for $5 \mathrm{~min}$. Then samples were ready for western blotting analysis. 


\section{Western blotting}

NP-40/Triton lysis buffer (10\% NP40, 1\% Triton $^{\text {TM }}$ X-100, $150 \mathrm{mM} \mathrm{NaCl}, 1 \mathrm{mM}$ EDTA, $50 \mathrm{mM}$ Tris, pH=8.0) containing proteinase inhibitors and phosphatase inhibitors was used to lyse both cultured cells and frozen brain tissue. Surface protein was extracted using Pierce ${ }^{\circledR}$ Cell Surface Protein Isolation Kit (Thermal Fisher Scientific, 89881) in transfected HEK-293T cells, and using ProteoExtract ${ }^{\mathrm{TM}}$ Native Membrane Protein Extraction Kit (Calbiochem, 444810) in brain tissue based on instructions. For transfected cells, proteins were extracted 36 hours after transfection. Cell and tissue lysate were lysed by ultrasound using an ultrasonic cleaner filled with icecold water for $30 \mathrm{~min}$. After incubated on ice for $1 \mathrm{~h}$, lysate was centrifuged at $13000 \mathrm{~g}$ for $30 \mathrm{~min}$ and supernatant was collected. Protein concentration was measured using Pierce ${ }^{\mathrm{TM}}$ Rapid Gold BCA Protein Assay Kit. 30-50 $\mu \mathrm{g}$ of total protein was loaded and separated proteins were transferred to Nitrocellulose membranes. Membranes were blocked with $5 \%$ BSA and $2.5 \%$ goat serum in Tris buffered saline (TBS, pH=7.4) at room temperature for $2 \mathrm{~h}$, and incubated with primary antibodies in TBS with $0.05 \%$ Tween (TBS-T) at room temperature for $2 \mathrm{~h}$. Then membranes were incubated with secondary antibodies in TBS-T for $1 \mathrm{~h}$ at room temperature for $1 \mathrm{~h}$ for detection. Blots were developed with ImageQuant LAS 4000 imaging system. Band intensity was quantified using ImageJ software and normalized with appropriate loading controls.

\section{Electrophysiology}

Whole cell currents were recorded using an Axopatch 200B amplifier. Data were digitized at 10 or $20 \mathrm{kHz}$ and digitally filtered offline at $1 \mathrm{kHz}$. Patch electrodes were pulled from borosilicate glass and fire-polished to a resistance of $\sim 3 \mathrm{M} \Omega$ when filled with internal solutions. Series resistance $\left(R_{s}\right)$ was compensated up to $90 \%$ to reduce series resistance errors to $<5 \mathrm{mV}$. Cells in which $\mathrm{R}_{\mathrm{s}}$ was $>10 \mathrm{M} \Omega$ were discarded (Du et al., 2009b). For heterologous expression, transfected HEK-293 cells were identified by GFP fluorescence. TRPM2 current 
recording in transfected HEK-293T cells was performed as we previously reported (Du et al., 2009a, b). TRPM2 and NMDAR currents recordings from cultured neurons were performed using SCSF as extracellular solution as we previously reported (Zeng et al., 2010). In brief, for TRPM2 current recordings, voltage stimuli lasting $250 \mathrm{~ms}$ were delivered at 1-s intervals, with voltage ramps ranging from -100 to $+100 \mathrm{mV}$ at holding potential of $0 \mathrm{mV}$ to elicited currents. For NMDA current recordings, a gap-free protocol at holding potential of $-80 \mathrm{mV}$ was applied to elicit NMDA currents upon agonist stimulation. A fast perfusion system was used to exchange extracellular solutions and to deliver agonists and antagonists to the cells, with a complete solution exchange achieved in about 1-3 s (Jiang et al., 2005).

Normal Tyrode solution contained (mM): $145 \mathrm{NaCl}, 5 \mathrm{KCl}, 2 \mathrm{CaCl}_{2}, 10$ HEPES, 10 glucose, osmolarity=290-320 $\mathrm{mOsm} / \mathrm{Kg}$, and $\mathrm{pH}=7.4$ was adjusted with $\mathrm{NaOH}$. Extracellular solution for current recording in neuron, the aCSF, solution contained (mM): $124 \mathrm{NaCl}, 2.5 \mathrm{KCl}, 2 \mathrm{MgSO}_{4}, 2 \mathrm{CaCl}_{2}, 1.2 \mathrm{NaH}_{2} \mathrm{PO}_{4}, 24 \mathrm{NaHCO}_{3}, 5 \mathrm{HEPES}, 12.5$ glucose, osmolarity $=300-310 \mathrm{mOsm} / \mathrm{Kg}$, with $\mathrm{pH}=7.4$ adjusted with $\mathrm{NaOH}$. For oxygen-glucose-deprivation (OGD) solution, glucose was eliminate from extracellular solution, and the solution was saturated with nitrogen $\left(\mathrm{N}_{2}\right)$ bubbling for 30 min before the experiments.

The internal pipette solution for whole cell current recordings of TRPM2 contained (in mM): 135 Csmethanesulfonate $\left(\mathrm{CsSO}_{3} \mathrm{CH}_{3}\right), 8 \mathrm{NaCl}, 0.5 \mathrm{CaCl}_{2}, 1 \mathrm{EGTA}$, and $10 \mathrm{HEPES}$, with $\mathrm{pH}$ adjusted to 7.2 with $\mathrm{CsOH}$. Free $\left[\mathrm{Ca}^{2+}\right]$ i buffered by EGTA was $100 \mathrm{nM}$ calculated using Max chelator (Du et al., 2009b). ADPR $200 \mu \mathrm{M}$ was included in the pipette solution for most experiments. The intracellular pipette solution to test the effects of OGD on TRPM2 currents in neuron was adjusted to sub-optimal condition, containing (in mM) $135 \mathrm{CsCH}_{3} \mathrm{SO}_{4}, 8 \mathrm{NaCl}$, $0.01 \mathrm{CaCl}_{2}, 1 \mathrm{MgCl}_{2}, 10$ HEPES (pH 7.2) and $10 \mu \mathrm{M}$ ADPR.

The intracellular solution for NMDAR current recording contained (mM): $110 \mathrm{~K}-\mathrm{ASP}, 20 \mathrm{KCl}, 1 \mathrm{MgSO}$, $0.05 \mathrm{mM}$ EGTA-K+, 0.1 GTP, 5 ATP-Mg2, 10 HEPES, osmolarity=275-285 mOsm/Kg, pH=7.2 adjusted with $\mathrm{KOH}$. For the experiments using cells pretreated with the disrupting peptides TAT-SC and TAT-EE $E_{3}, 10 \mu \mathrm{M}$ TAT-SC or TAT-EE 3 
was included in the pipette solution, and at least 10 min was allowed for achieving intracellular equilibration of TAT-SC or TAT-EE 3 before current recording.

For current recordings in neurons, tetrodotoxin $(0.5 \mu \mathrm{M})$ was included in the external solution to block voltagegated $\mathrm{Na}^{+}$current, and $10 \mu \mathrm{M}$ nifedipine was used to block voltage-gated $\mathrm{Ca}^{2+}$ currents for recording TRPM2 currents.

\section{Immunofluorescence staining}

Brains harvested from mice were frozen at $-80^{\circ} \mathrm{C}$ prior to use, and was mounted in Fisher Healthcare ${ }^{\mathrm{TM}}$ TissuePlus $^{\mathrm{TM}}$ O.C.T. Compound (Thermal Fisher Scientific, 23-730-571) prior to cutting. Brains were cut into sagittal slices at a thickness of 6 to $8 \mu \mathrm{m}$, mounted to Superfrost $\AA^{\circledR}$ Plus Microscope Slides (Thermal Fisher Scientific, 12550-15), and frozen at $-80^{\circ} \mathrm{C}$ for future use. Prior to staining, slides were taken to room temperature for at least 30 min allowing for dehydration. Slices were fixed in 10\% formaldehyde for 15 min following washing using PBS for 3 times, and incubated in blocking solution containing 5\% BSA, 15\% goat serum and 1\% Triton X-100 at room temperature for $2 \mathrm{~h}$. Primary antibodies were diluted as described previously in TBS-T containing $15 \%$ goat serum. Slices were incubated with primary antibodies for at least $12 \mathrm{~h}$ at $4{ }^{\circ} \mathrm{C}$ following washing using PBS for 3 times, and incubated with secondary antibodies at room temperature for $2 \mathrm{~h}$. Then slices were washed using PBS for 3 times, and mounted using Prolong® Gold anti-fade reagent with DAPI. Slices were kept at $4{ }^{\circ} \mathrm{C}$ before taking pictures. TUNEL staining was performed based on the instruction of kit.

\section{Data analysis}

All data are expressed as mean \pm SEM. For two groups' comparison, statistical significance was determined using Student's t-test. For multiple groups' comparison, statistical significance was determined using one-way or twoway analysis of variance (ANOVA) followed by Bonferroni posttest. $\mathrm{P}<0.05$ was regarded as significant. 
Table S1. Primers for subcloning, mutagenesis and genotyping

\begin{tabular}{|c|c|c|c|}
\hline Application & Genes & Primers & Primer sequences $\left(5^{\prime}-3^{\prime}\right)$ \\
\hline \multirow[t]{9}{*}{ Subcloning } & \multirow{3}{*}{$\begin{array}{l}\text { Trpm2 C } \\
\text { terminal part }\end{array}$} & $\mathrm{F}$ & CTCGAATTCTGAAGGAGAACTACCTCCAGAAC \\
\hline & & & \\
\hline & & $\mathrm{R}$ & GATCTAGATTAGGTCTTGTGGTTCGCATAGAGTG \\
\hline & \multirow{3}{*}{$\begin{array}{l}\text { GluN2a C } \\
\text { terminal part }\end{array}$} & $\mathrm{F}$ & CGGAATTCCGACACTCTTCTACTGGAAG \\
\hline & & & \\
\hline & & $\mathrm{R}$ & TGCTCTAGAGCTTAAACATCAGATTCGATACTAGG \\
\hline & \multirow{3}{*}{$\begin{array}{l}\text { GluN2b C } \\
\text { terminal part }\end{array}$} & $\mathrm{F}$ & CGGAATTCCGTCATCACCTTCATCTGTGAG \\
\hline & & & \\
\hline & & $\mathrm{R}$ & TGCTCTAGAGCACCTTAACCTCTCTCTCTTC \\
\hline \multirow[t]{23}{*}{ Mutagenesis } & \multirow[t]{2}{*}{ Trpm2 1-727 } & $\mathrm{F}$ & CAAGGACATGTAGTTTGTGTC \\
\hline & & $\mathrm{R}$ & GACACAAACTACATCATGTCCTTG \\
\hline & \multirow[t]{2}{*}{ Trpm2 1-679 } & $\mathrm{F}$ & TGGCGCTGGCGTAGTAGTATG \\
\hline & & $\mathrm{R}$ & TCATACTACTACGCCAGCGCC \\
\hline & \multirow[t]{2}{*}{ Trpm2 1-631 } & $\mathrm{F}$ & ATTTGGGCCATTGTCTAGAACCGT \\
\hline & & $\mathrm{R}$ & ACGGTTCTAGACAATGGCCCAAATG \\
\hline & \multirow[t]{2}{*}{ Trpm $21-570$} & $\mathrm{~F}$ & TGCTGGGGGAATTCACGCAG \\
\hline & & $\mathrm{R}$ & TGCGTGAATTCCCCCAGCAG \\
\hline & \multirow[t]{2}{*}{ Trpm2 1-664 } & $\mathrm{F}$ & TGAAGGAACTGTCCTAGGAGGAGGAG \\
\hline & & $\mathrm{R}$ & TCCTCCTCCTAGGACAGTTCCTTCAG \\
\hline & \multirow{3}{*}{$\begin{array}{l}\text { Trpm2 } \\
\text { deletion }\end{array}$} & $\mathrm{F}$ & AAGATCCTGAAGGAACTGTCCAAGTATGAGCACAGAGCCATC \\
\hline & & & \\
\hline & & $\mathrm{R}$ & GATGGCTCTGTGCTCATACTTGGACAGTTCCTTCAGGATCTT \\
\hline & \multirow[t]{2}{*}{$\operatorname{Trpm} 2 \mathrm{QEE}$} & $\mathrm{F}$ & AAGGAACTGTCCAAGCAGCAGGAGGACACGGAC \\
\hline & & $\mathrm{R}$ & TCCGTGTCCTCCTGCTGCTTGGACAGTTCCTTC \\
\hline & \multirow[t]{2}{*}{$\operatorname{Trpm} 2 \mathrm{EQE}$} & $\mathrm{F}$ & ACACGGACAGCTCGCAGCAGATGCTGGCG \\
\hline & & $\mathrm{R}$ & CGCCAGCATCTGCTGCGAGCTGTCCGTGTC \\
\hline & \multirow[t]{2}{*}{$\operatorname{Trpm} 2 \mathrm{EEQ}$} & $\mathrm{F}$ & TGGCGCTGGCGCAGCAGTATGAGCACAGAG \\
\hline & & $\mathrm{R}$ & TCTGTGCTCATACTGCTGCGCCAGCGCCAG \\
\hline & \multirow[t]{2}{*}{ GluN2a 1-1053 } & $\mathrm{F}$ & ACCTTCATCTGGTAGCACCTCTTCTAC \\
\hline & & $\mathrm{R}$ & TAGAAGAGGTGCTACCAGATGAAGGTG \\
\hline & \multirow[t]{2}{*}{ GluN2a 1-1047 } & $\mathrm{F}$ & ACCTTCATCTGTTAGCATCTGTTCTATTG \\
\hline & & $\mathrm{R}$ & AATAGAACAGATGCTAACAGATGAAGGTG \\
\hline \multirow[t]{8}{*}{ Genotyping } & \multirow[t]{2}{*}{ Cre } & $\mathrm{F}$ & GATATCTCACGTACTGACGG \\
\hline & & $\mathrm{R}$ & TGACCAGAGTCATGGTTAGC \\
\hline & \multirow[t]{3}{*}{ Trpm2 loxp } & $\mathrm{F}$ & GGCTCTGCCTCATCCCCAGAATC \\
\hline & & $\mathrm{R}$ & CCGGATACAGATGCAGGATGCTG \\
\hline & & $\mathrm{R}$ & CTGAAGGTCCTGAGTTTGAATCCCA \\
\hline & \multirow[t]{3}{*}{ TRPM2-KO } & $\mathrm{F}$ & CTTGGGTTGCAGTCATATGCAGGC \\
\hline & & $\mathrm{R}$ & GCCCTCACCATCCGCTTCACGATG \\
\hline & & $\mathrm{R}$ & GCCACACGCGTCACCTTAATATGC \\
\hline
\end{tabular}




\section{REFERENCE LIST (For START * METHOD)}

Chiang, T., Messing, R.O., and Chou, W.H. (2011). Mouse model of middle cerebral artery occlusion. Journal of visualized experiments : JoVE.

Du, J., Xie, J., and Yue, L. (2009a). Intracellular calcium activates TRPM2 and its alternative spliced isoforms. Proceedings of the National Academy of Sciences 107, 7239-7244.

$\mathrm{Du}, \mathrm{J} ., \mathrm{Xie}, \mathrm{J}$., and Yue, L. (2009b). Modulation of TRPM2 by acidic $\mathrm{pH}$ and the underlying mechanisms for $\mathrm{pH}$ sensitivity. J Gen Physiol 134, 471-488.

Du, J., Xie, J., Zhang, Z., Tsujikawa, H., Fusco, D., Silverman, D., Liang, B., and Yue, L. (2010). TRPM7-mediated $\mathrm{Ca}^{2+}$ signals confer fibrogenesis in human atrial fibrillation. Circulation research 106, 992-1003.

Jiang, J., Li, M., and Yue, L. (2005). Potentiation of TRPM7 inward currents by protons. J Gen Physiol 126, 137150.

Liu, F., and McCullough, L.D. (2014). The middle cerebral artery occlusion model of transient focal cerebral ischemia. Methods in molecular biology 1135, 81-93.

Longa, E.Z., Weinstein, P.R., Carlson, S., and Cummins, R. (1989). Reversible middle cerebral artery occlusion without craniectomy in rats. Stroke; a journal of cerebral circulation 20, 84-91.

Miller, B.A., Wang, J., Hirschler-Laszkiewicz, I., Gao, E., Song, J., Zhang, X.Q., Koch, W.J., Madesh, M., Mallilankaraman, K., Gu, T., et al. (2013). The second member of transient receptor potential-melastatin channel family protects hearts from ischemia-reperfusion injury. American journal of physiology Heart and circulatory physiology 304, H1010-1022.

Perraud, A.L., Schmitz, C., and Scharenberg, A.M. (2003). TRPM2 Ca2+ permeable cation channels: from gene to biological function. Cell calcium 33, 519-531.

Ren, M., Senatorov, V.V., Chen, R.W., and Chuang, D.M. (2003). Postinsult treatment with lithium reduces brain damage and facilitates neurological recovery in a rat ischemia/reperfusion model. Proceedings of the National Academy of Sciences of the United States of America 100, 6210-6215.

Thompson, R.J., Zhou, N., and MacVicar, B.A. (2006). Ischemia opens neuronal gap junction hemichannels. Science 312, 924-927.

Wu, L.J., Wu, G., Akhavan Sharif, M.R., Baker, A., Jia, Y., Fahey, F.H., Luo, H.R., Feener, E.P., and Clapham, D.E. (2012). The voltage-gated proton channel Hv1 enhances brain damage from ischemic stroke. Nature neuroscience 15, 565-573.

Yamamoto, S., Shimizu, S., Kiyonaka, S., Takahashi, N., Wajima, T., Hara, Y., Negoro, T., Hiroi, T., Kiuchi, Y., Okada, T., et al. (2008). TRPM2-mediated Ca2+influx induces chemokine production in monocytes that aggravates inflammatory neutrophil infiltration. Nature medicine 14, 738-747.

Zeng, H., Guo, M., Martins-Taylor, K., Wang, X., Zhang, Z., Park, J.W., Zhan, S., Kronenberg, M.S., Lichtler, A., Liu, H.X., et al. (2010). Specification of region-specific neurons including forebrain glutamatergic neurons from human induced pluripotent stem cells. PloS one 5, e11853. 
bioRxiv preprint doi: https://doi.org/10.1101/2021.07.29.454247; this version posted July 30, 2021. The copyright holder for this preprint (which was not certified by peer review) is the author/funder. All rights reserved. No reuse allowed without permission. 
Figure 1
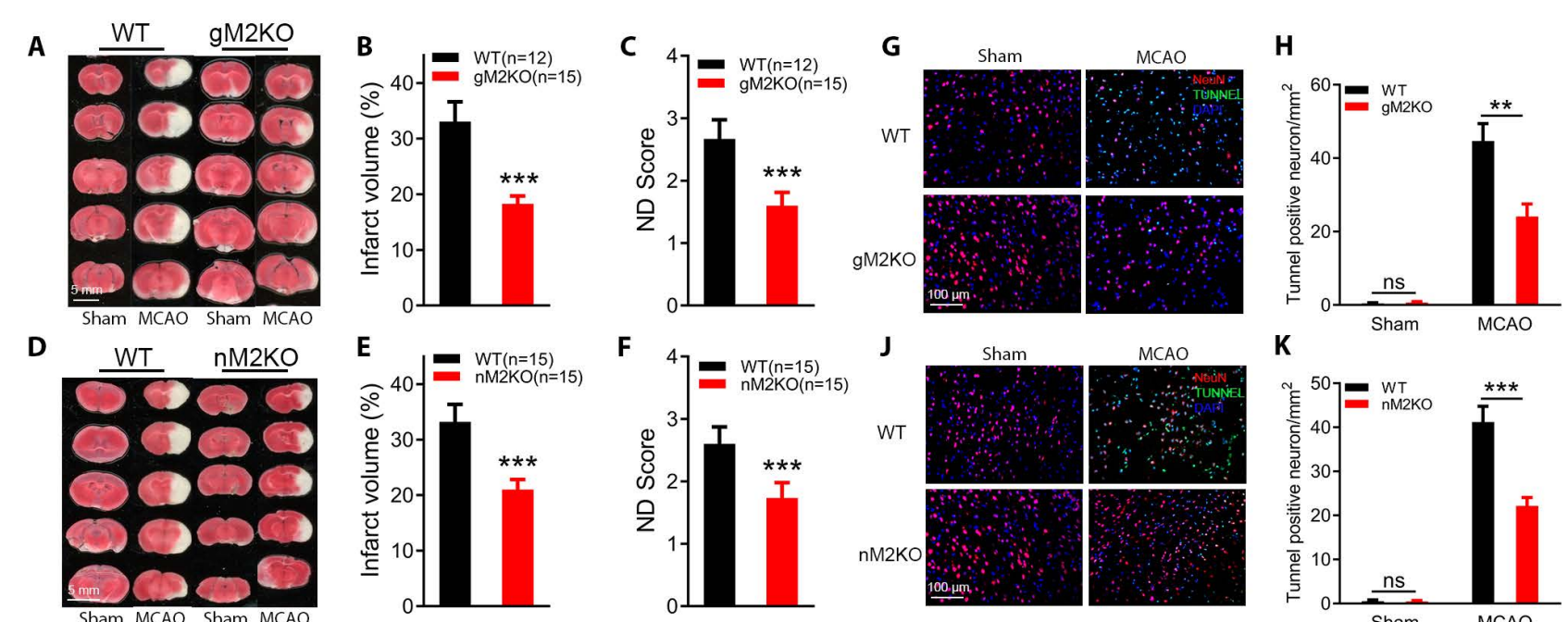

K
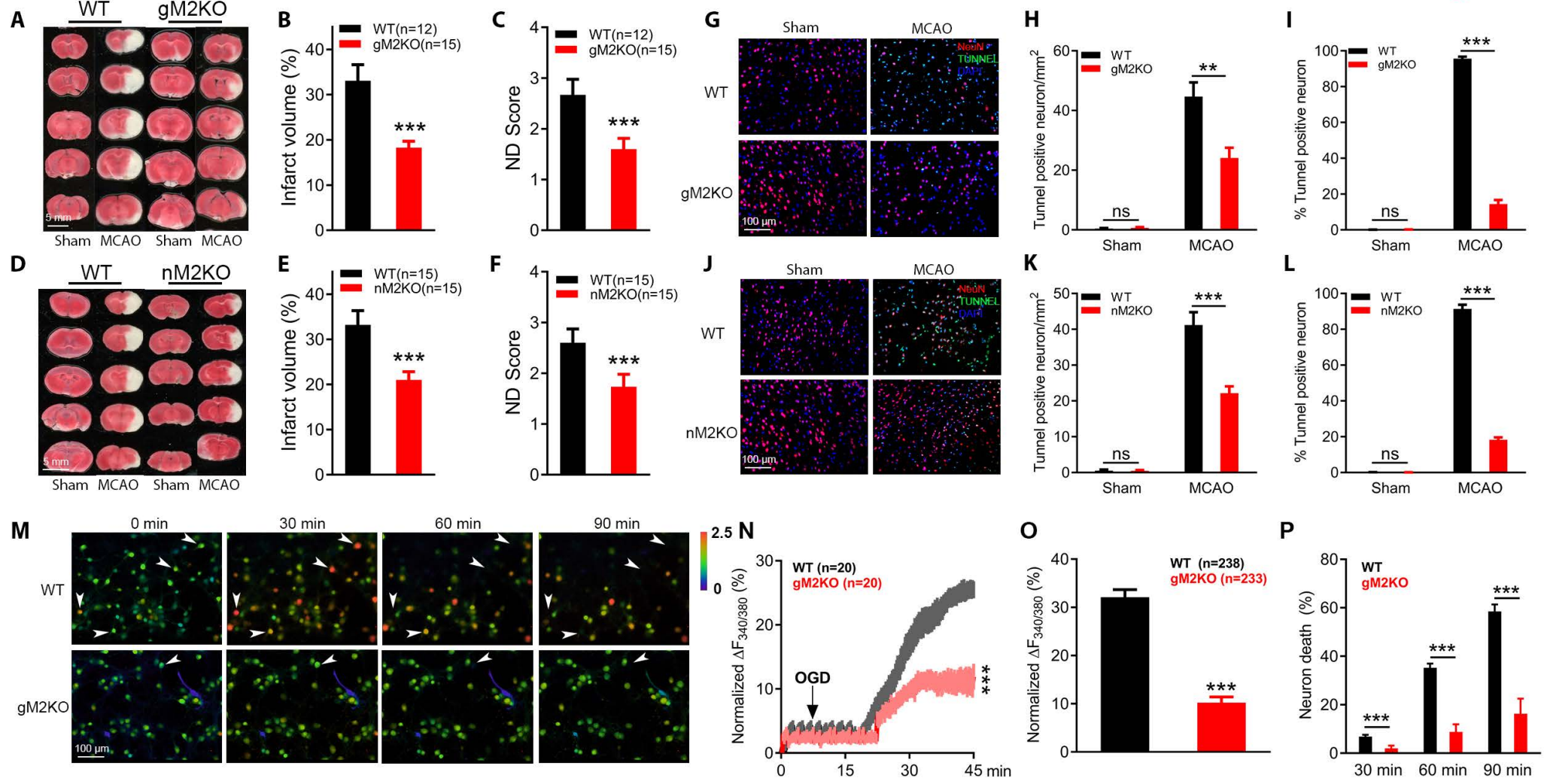

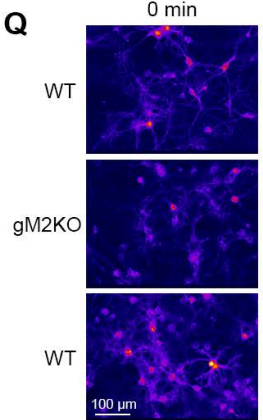

U

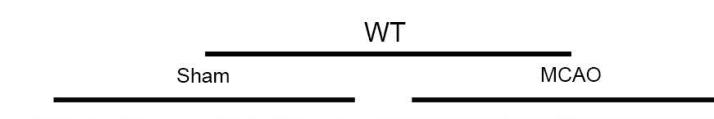

$170 \mathrm{kDa}$

$55 \mathrm{kDa}$
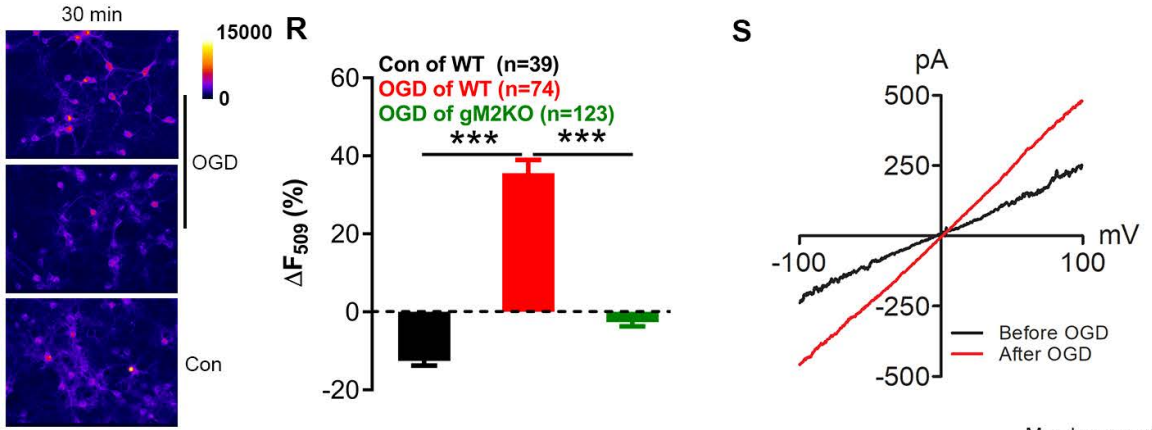

T
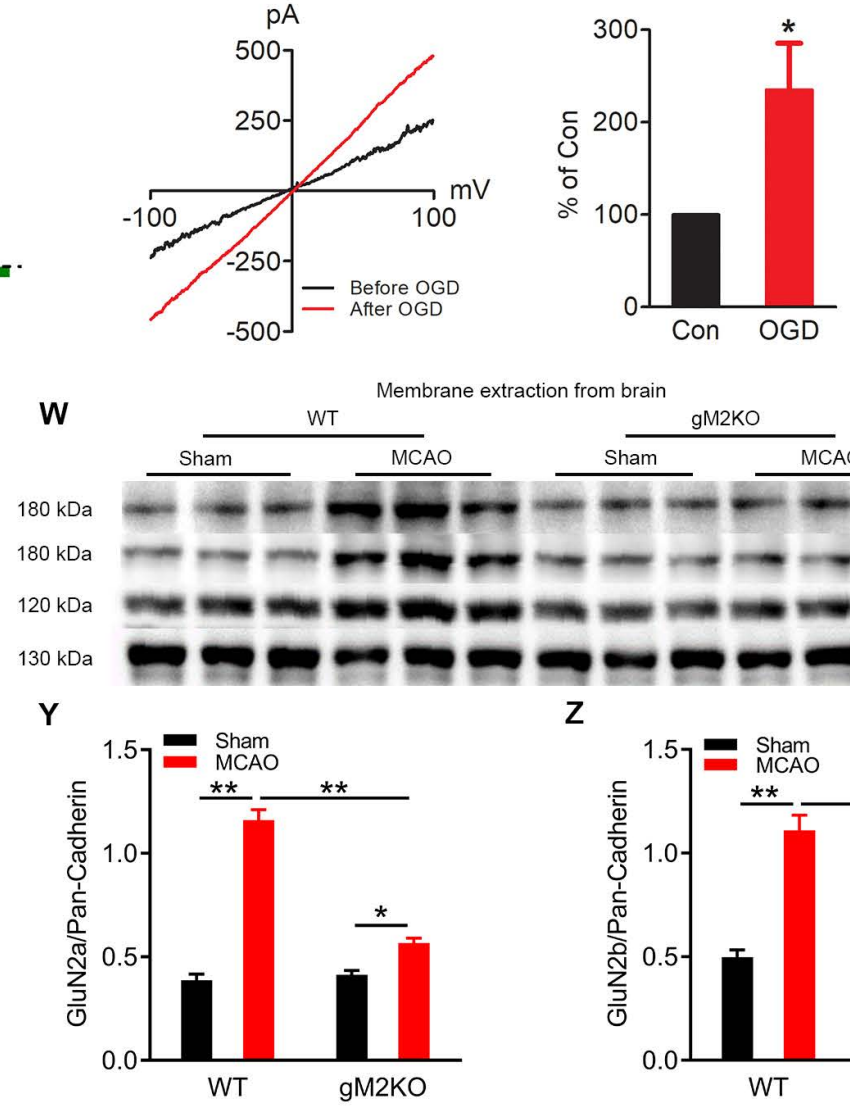

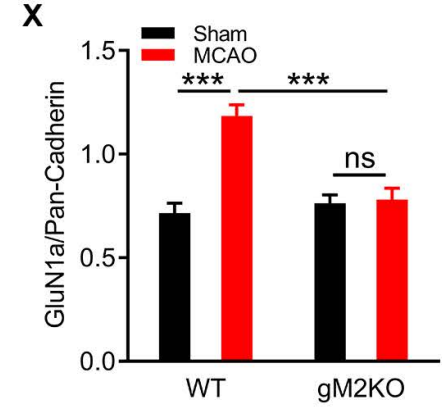

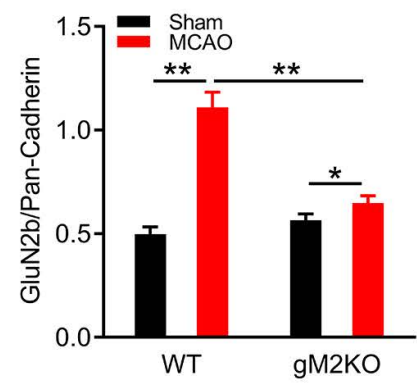


Figure 2

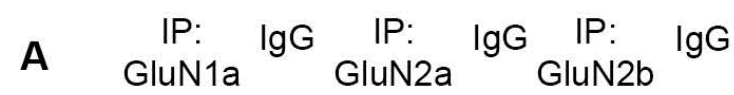
$180 \mathrm{kDa}$

B GluN1a,GluN2a and GluN2b

C GluN1a,GluN2a or GluN2b

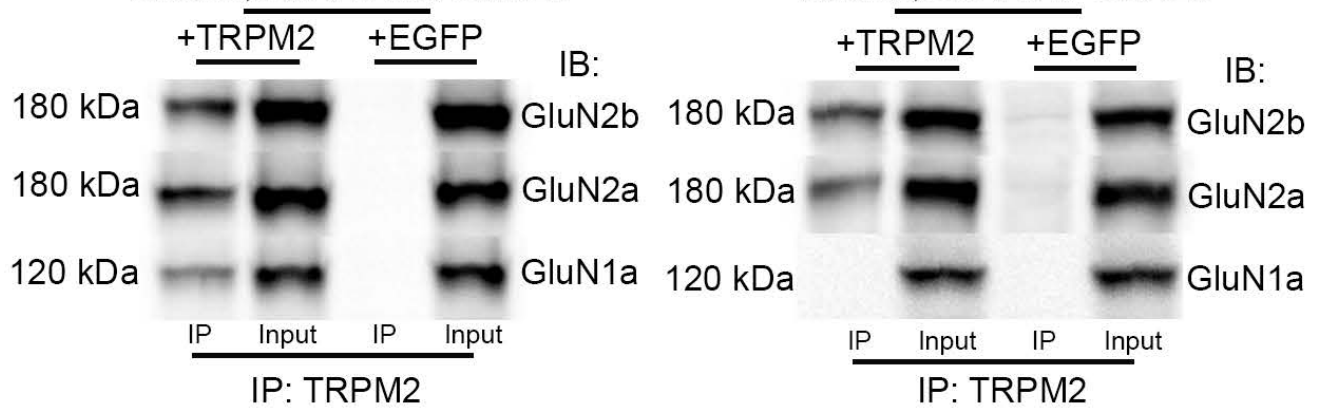

D

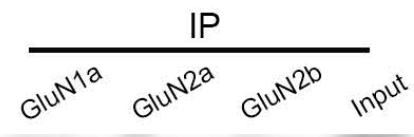

$180 \mathrm{kDa}$

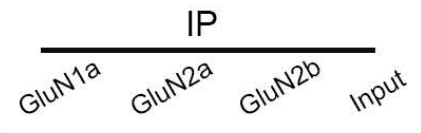

IB: TRPM2

WT

$\mathrm{gM} 2 \mathrm{KO}$

E

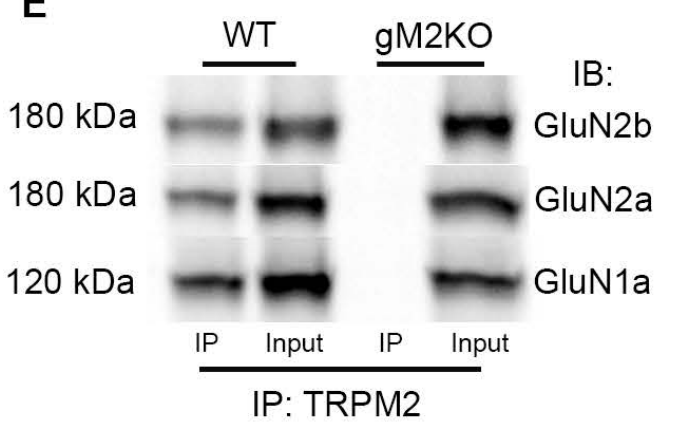

G
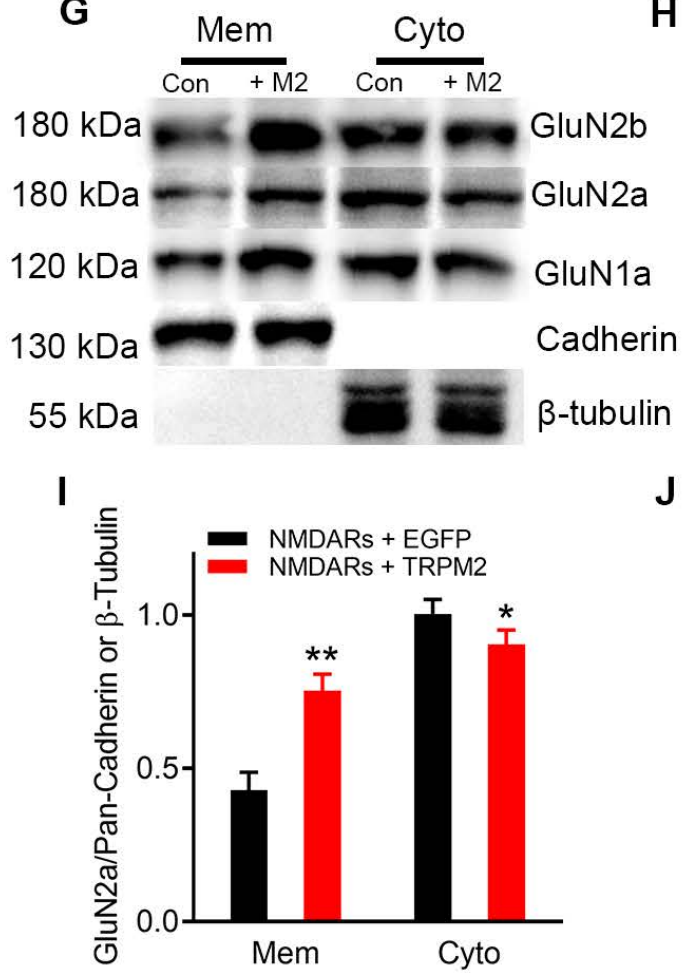

J

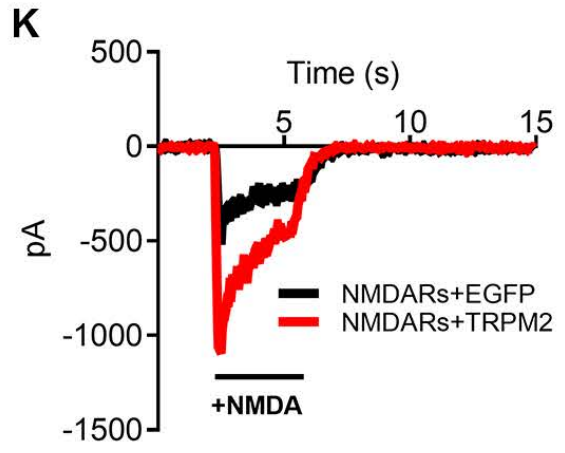

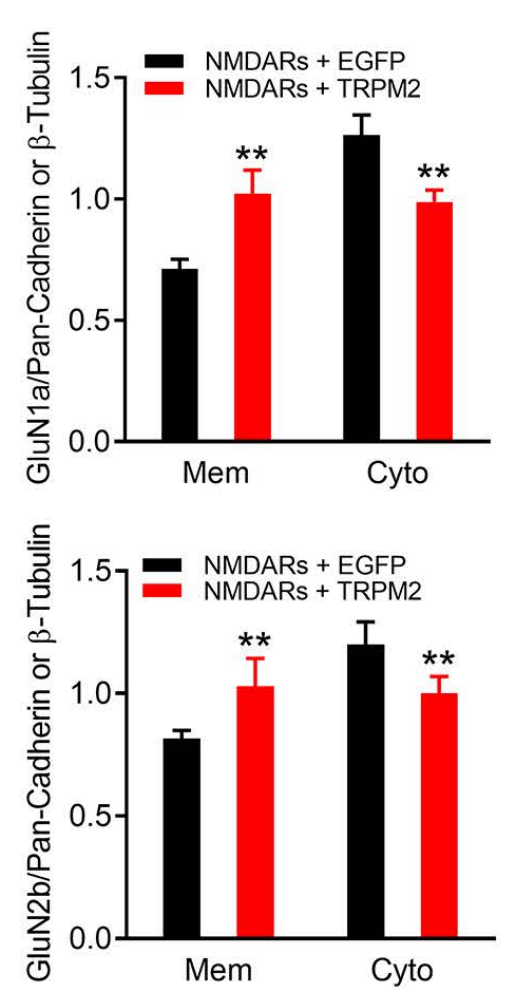

L

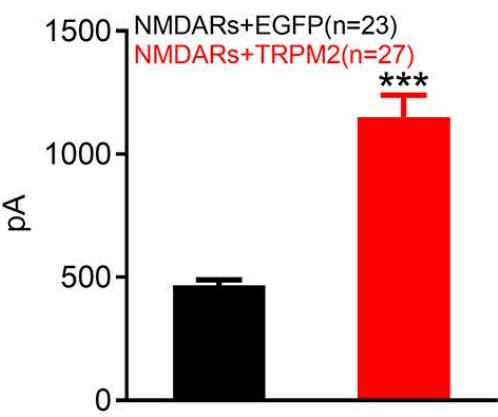


A

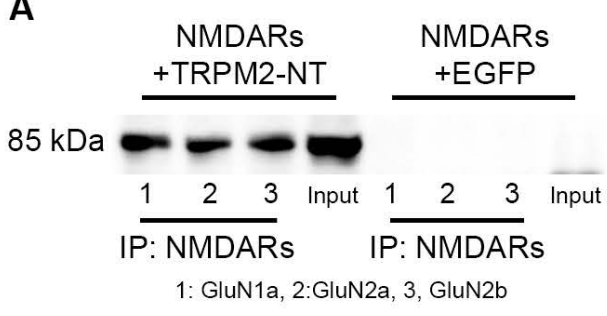

B $\begin{array}{cc}\text { NMDARs } & \text { NMDARs } \\ \text { +TRPM2-CT } & \text { +EGFP }\end{array}$

$55 \mathrm{kDa}$

$\frac{+ \text { TRPM2-CT }}{\text { +EGFP }}$

$\begin{array}{lllllll}1 \quad 2 & 3 & \text { Input } & 1 & 2 & 3 & \text { Input }\end{array}$

IP: NMDARs IP: NMDARs

1: GluN1a, 2:GluN2a, 3, GluN2b

C $\begin{array}{lllllllll}1 & 2 & 3 & 4 & \lg G & 1 & 2 & 3 & 4\end{array}$

$150 \mathrm{kDa}>$

$75 \mathrm{kDa}>\frac{\mathrm{IP}}{\mathrm{IP}: \text { TRPM2 }} \frac{--\mathrm{IBFP}}{\text { Input }}$

1: GluN2a- $\Delta C T$, 2: GluN2b- $\Delta C T$; 3: GluN2a-CT; 4: GluN2b-CT
D

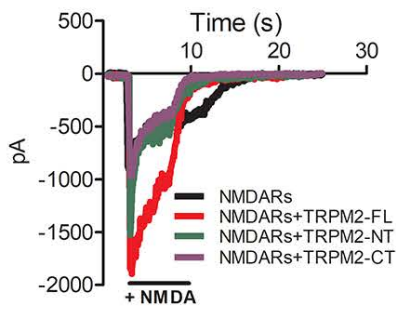

E

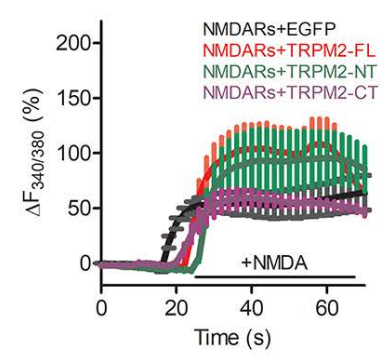

F TRPM2

IB: Flag

IB: GFP

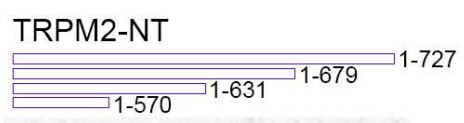

$75 \mathrm{kDa}>$

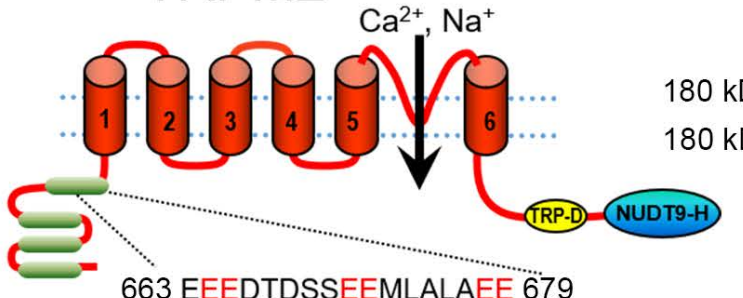

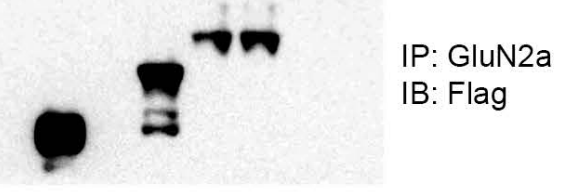

TRPM2-NT

Figure 3

$180 \mathrm{kDa}-\infty \mathrm{IB}:$ GluN2a $180 \mathrm{kDa}$ P: TRPM2
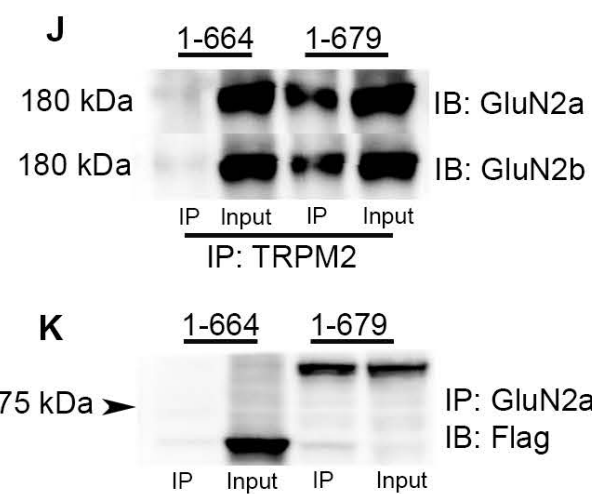

I

$75 \mathrm{kDa}>$

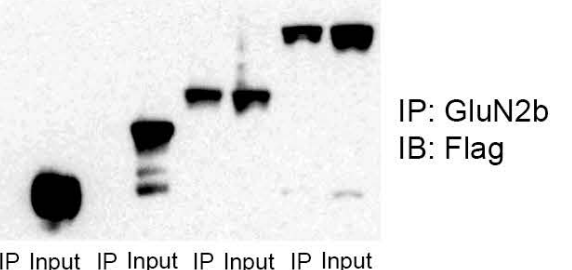

IP: GluN2b

IP Input IP Input
IP Input IP Input IP Input IP Input
N NMDARs+EGFP
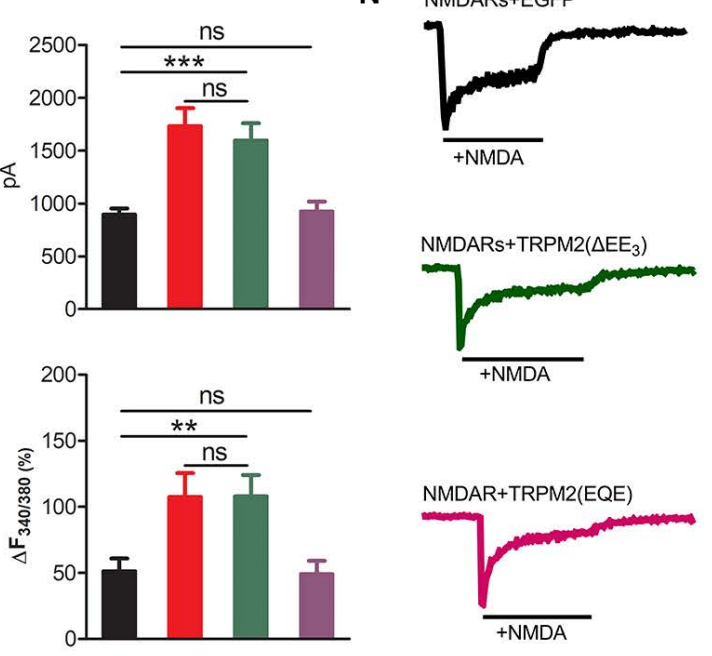

NMDARs+TRPM2(WT)

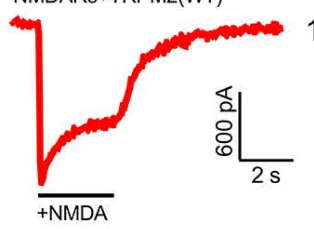

NMDAR+TRPM2(QEE)

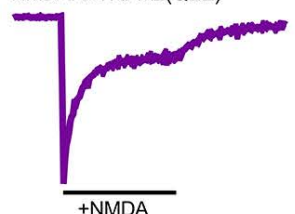

NMDAR+TRPM2(EQE)

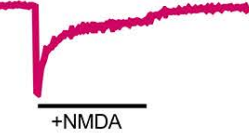

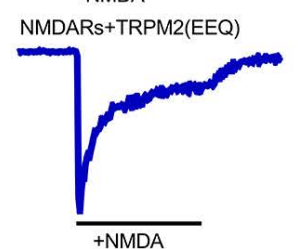

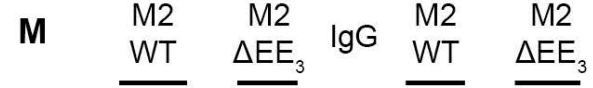

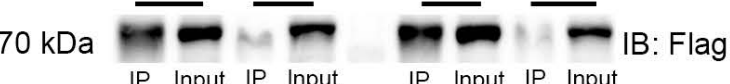

IP: Input IP Input IP Input IP Input

O QEE EQE EEQ (WT)

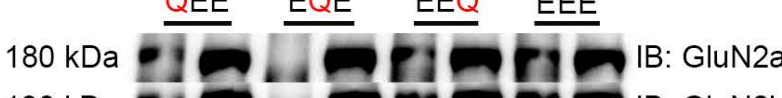
$180 \mathrm{kDa}$ e IP Input IP Input IP Input IP Input

$\mathbf{P}$

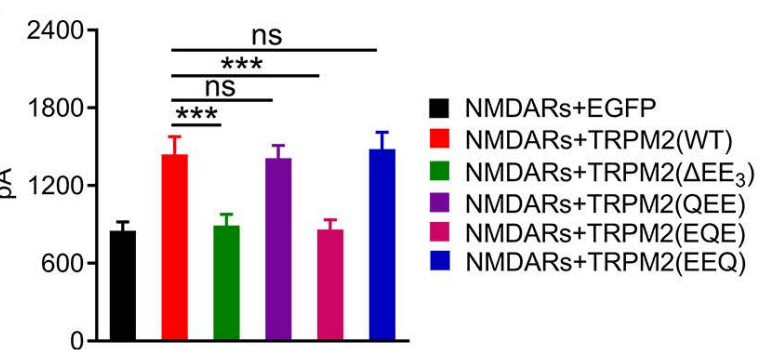




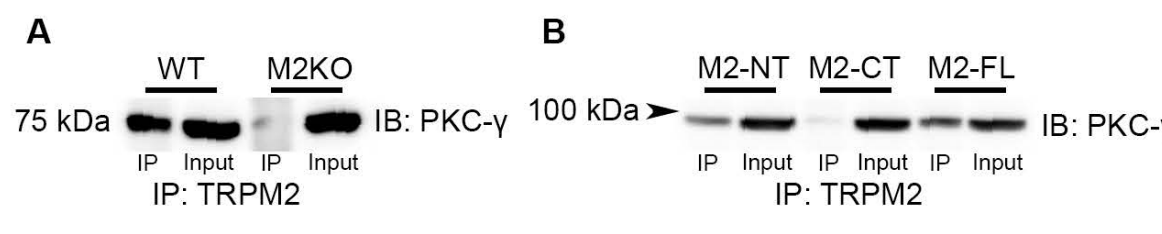

C

$75 \mathrm{kDa}$ Sham MCAO

$75 \mathrm{kDa}=\mathrm{PKC}-\mathrm{M}$ IIP: $170 \mathrm{kDa} \backsim \sim$ TRPM2 ITRPM2 $75 \mathrm{kDa}=-\mathrm{PKC}-\mathrm{Y}$ $55 \mathrm{kDa}$ WT gM2KO

E

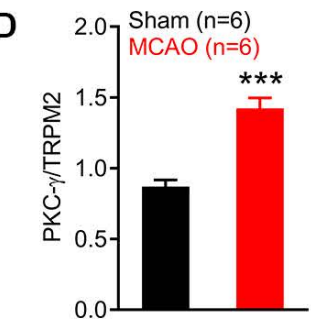

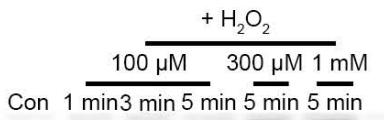

$75 \mathrm{kDa}=1$ min 3 min 5 min 5 min 5 min $170 \mathrm{kDa}$ $55 \mathrm{kDa}$

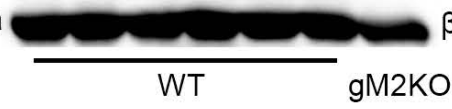

PKC-Y IP: TRPM2 ITRPM2 PK-tubulin Input WT $\quad$ gM2KO
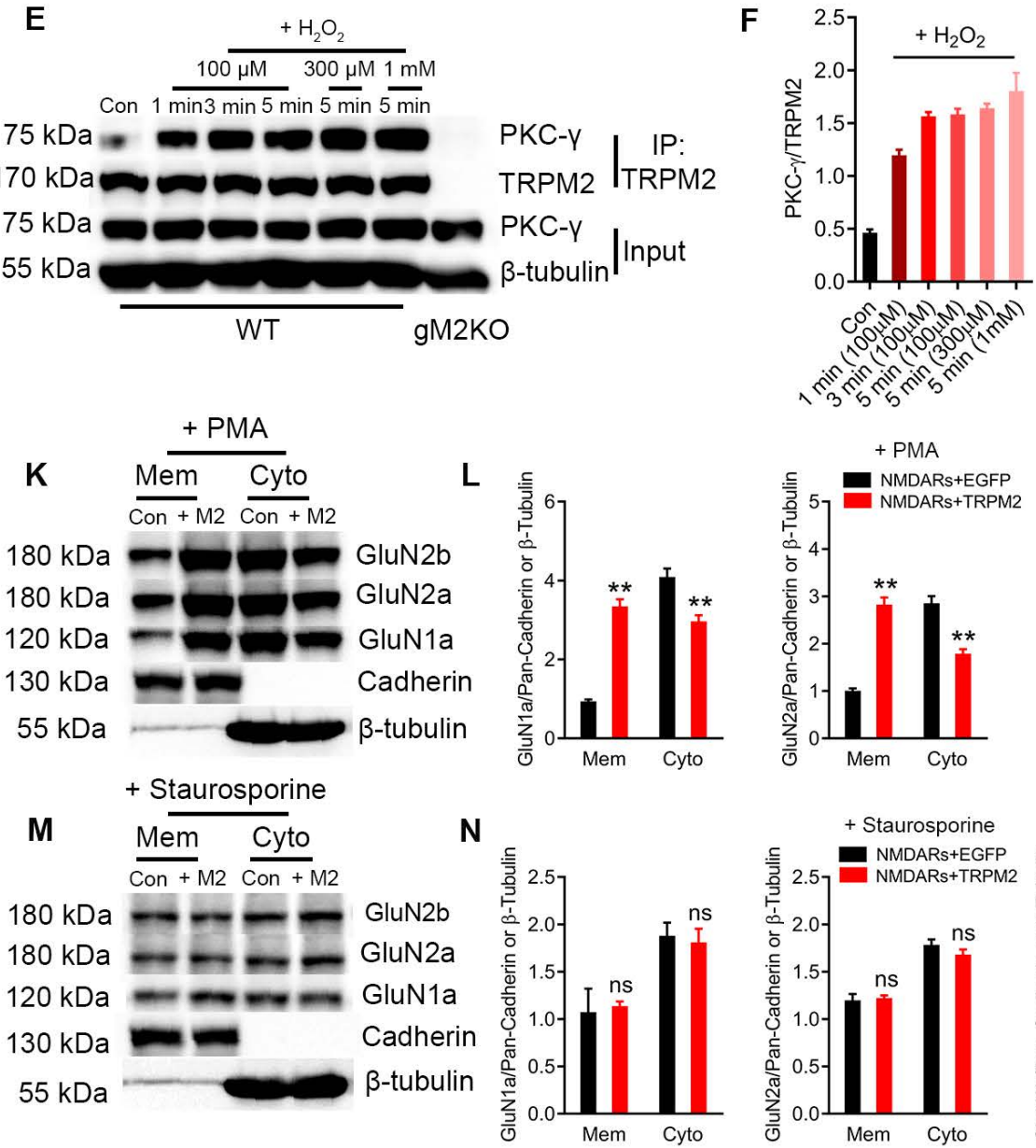

G $\frac{+ \text { PKC-y }}{\text { Mem }} \frac{\text { Cyto }}{\text { Con }+ \text { M2 } 2 \text { Con }+ \text { M2 }}$

$180 \mathrm{kDa}=-$ GluN2b $180 \mathrm{kDa}-\mathrm{m}=-$ GluN2a $120 \mathrm{kDa}=0$ GluN1a $130 \mathrm{kDa}=0 \quad$ Cadherin $55 \mathrm{kDa}$ Cadherin I $\frac{+ \text { PKC- } y \text {-DN }}{\text { Men }+ \text { M2 }} \frac{\text { Cyto }}{\text { Con }+ \text { M2 }}$

$180 \mathrm{kDa}=\sigma 0$ GluN2b $180 \mathrm{kDa}=\boldsymbol{m}=$ GluN2a $120 \mathrm{kDa}$ GluN1a $130 \mathrm{kDa}=0$ Cadherin $55 \mathrm{kDa}$ - $\beta$-tubulin
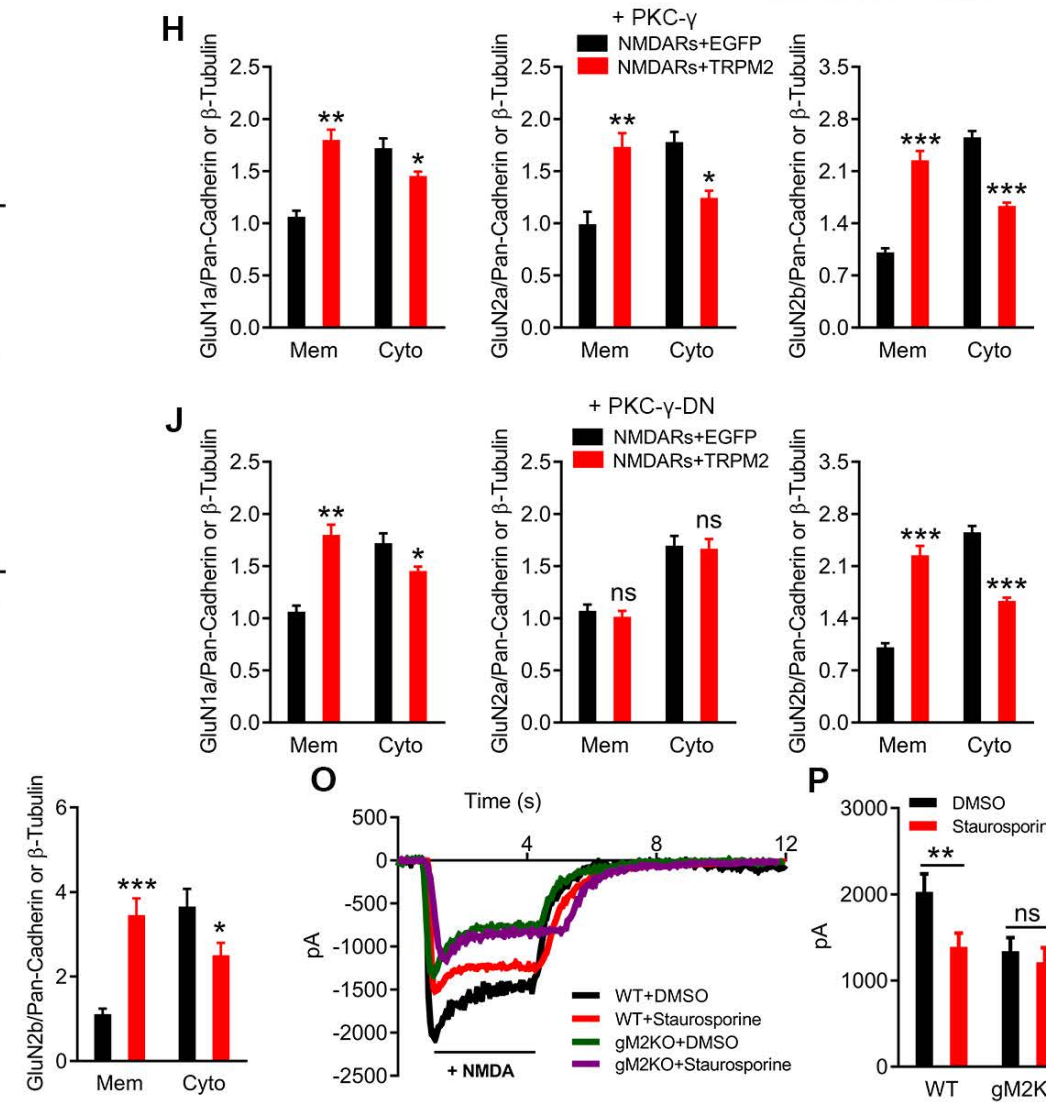

$\mathbf{P}$
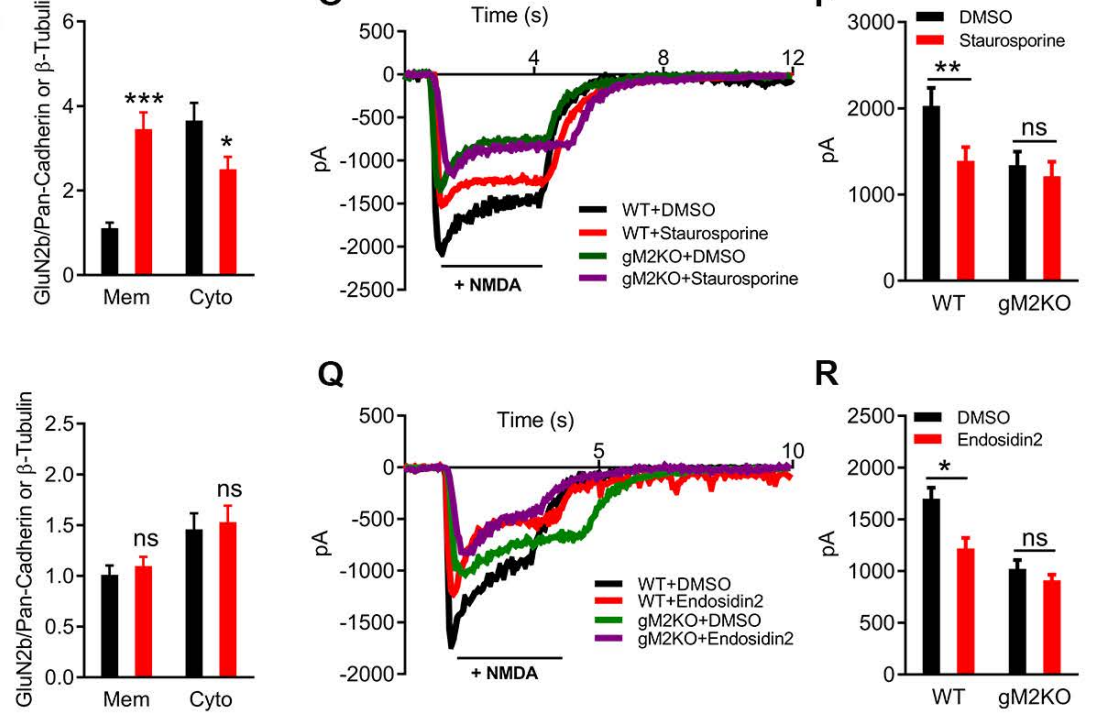

Q
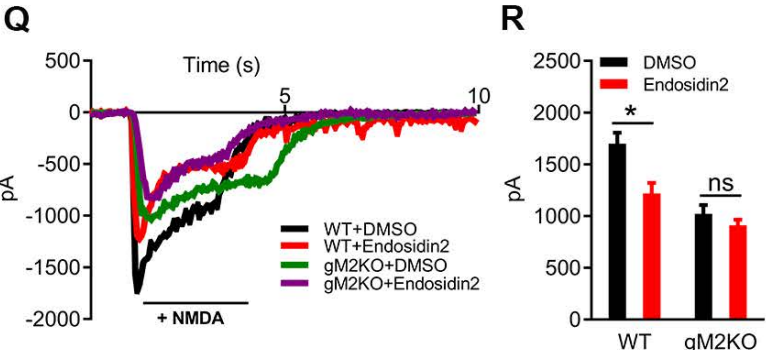
Figure 5

A

$$
\frac{W T}{\text { TAT TAT }} \frac{\text { WT }}{\text { TAT TAT }}
$$

-SC $-\mathrm{EE}_{3} \Delta \mathrm{EE}_{3}$ QEE EQE EEQ IgG $\quad-S C \quad-\mathrm{EE}_{3} \Delta \mathrm{EE}_{3}$ QEE EQE EEQ $170 \mathrm{kDa}=$ $180 \mathrm{kDa}$

B

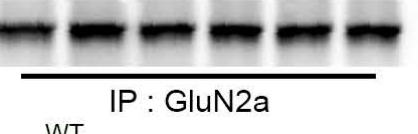

IB: Flag $\frac{\text { WT }}{\text { TAT TAT }} \frac{\text { WT }}{\text { TAT TAT }}$

$170 \mathrm{kDa}$

$180 \mathrm{kDa}=-\mathrm{m}=$

$-m-m$ IB: GluN2a

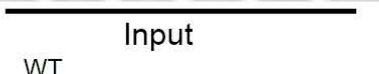

$-S C-E_{3} \triangle E_{3}$ QEE EQE EEQ

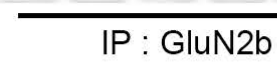 \\ IP: GluN2 \\ C

$$
\frac{\text { Mem }}{\text { Con }+ \text { M2 } 2 \text { Con }+ \text { M2 }}
$$

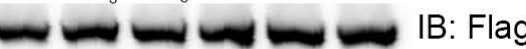

$180 \mathrm{kDa}=\mathrm{GluN2b}$

$m-\infty \rightarrow$ IB: GluN2b

$\mathrm{kDa} m-$ GluN2a $180 \mathrm{kDa} m$ GluN2a

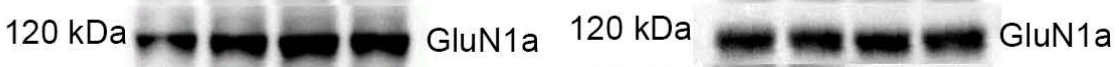
$130 \mathrm{kDa}$ Cadherin $130 \mathrm{kDa}$

Input

$55 \mathrm{kDa}$

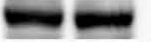

Cadherin $130 \mathrm{kDa}$

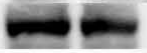

Cadherin

E
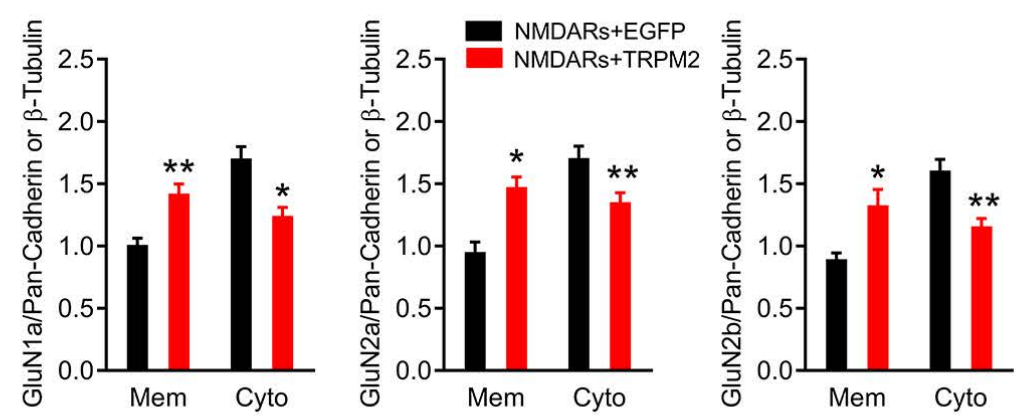

F
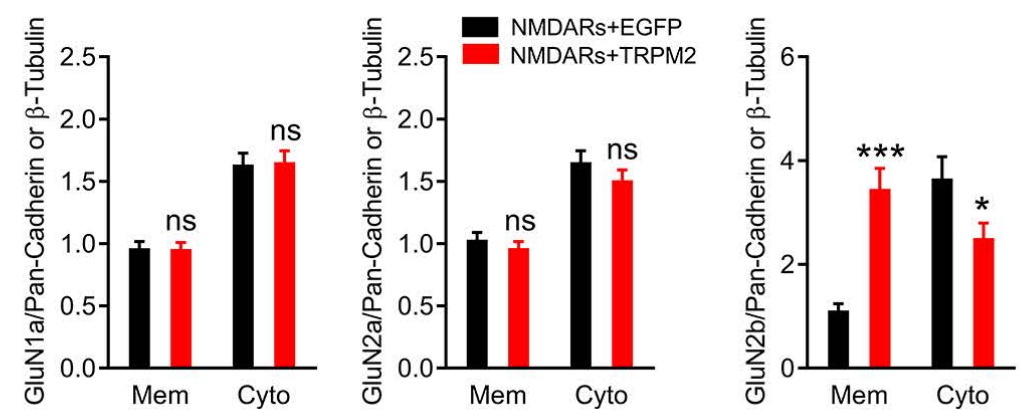

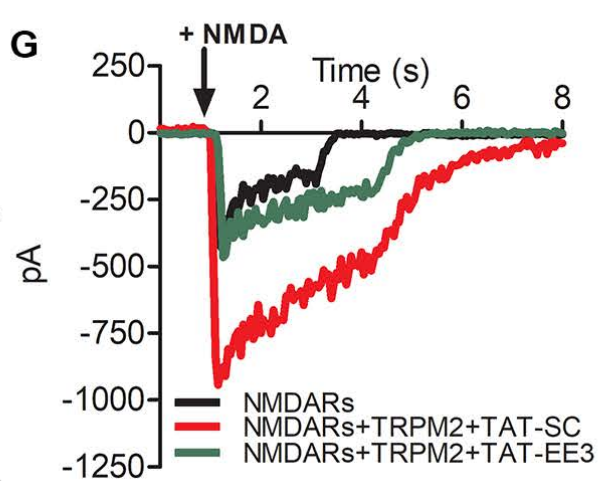

$\mathrm{H}$

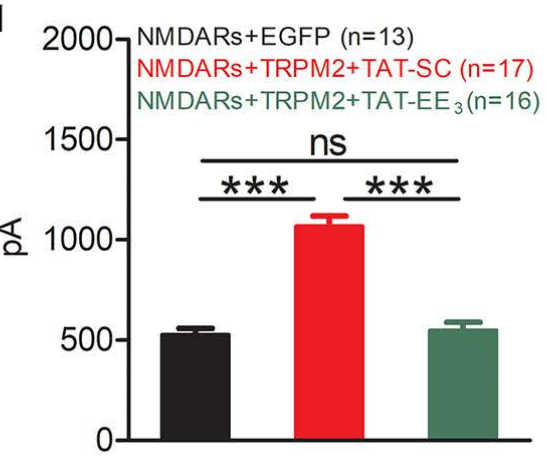

I

WT+TAT-SC

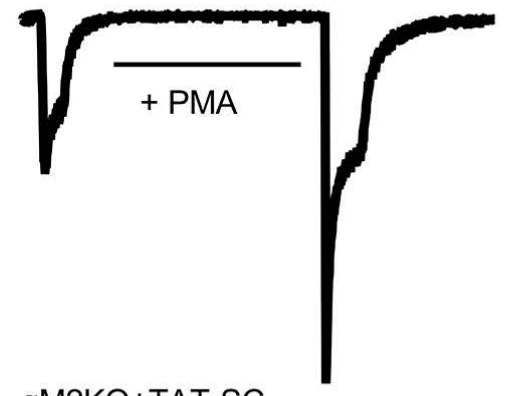

gM2KO+TAT-SC

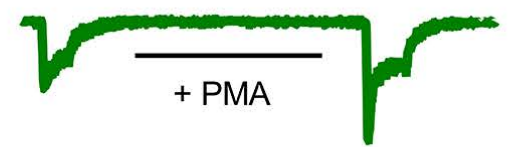

J

WT+TAT-EE 3

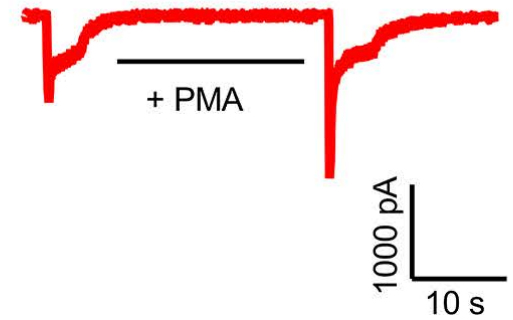

gM2KO+TAT-EE

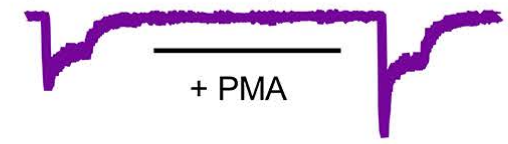

K
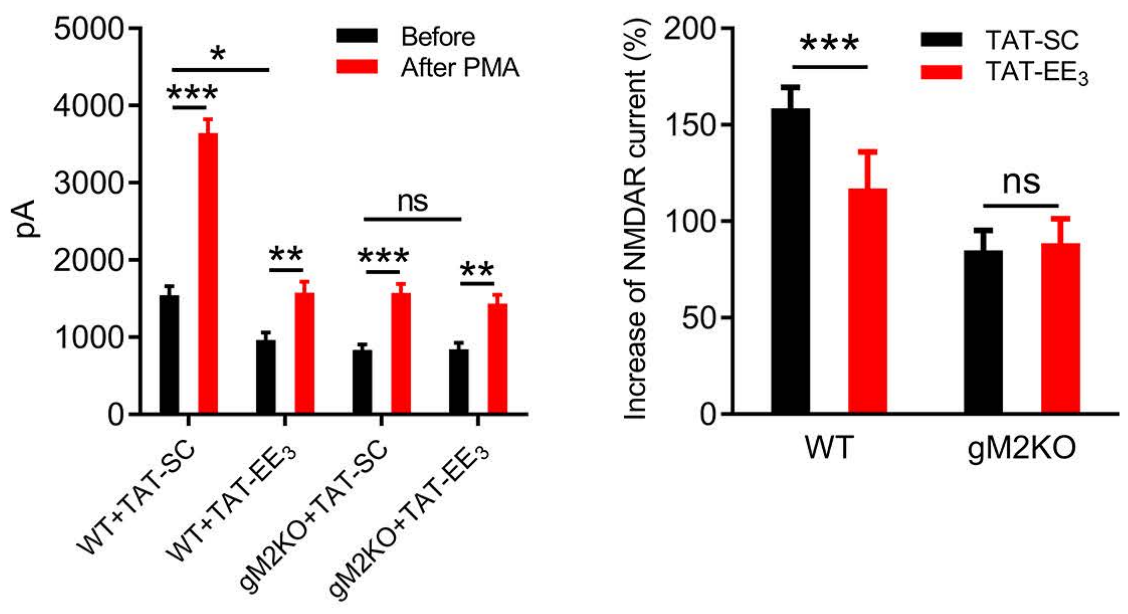
Figure 6

A
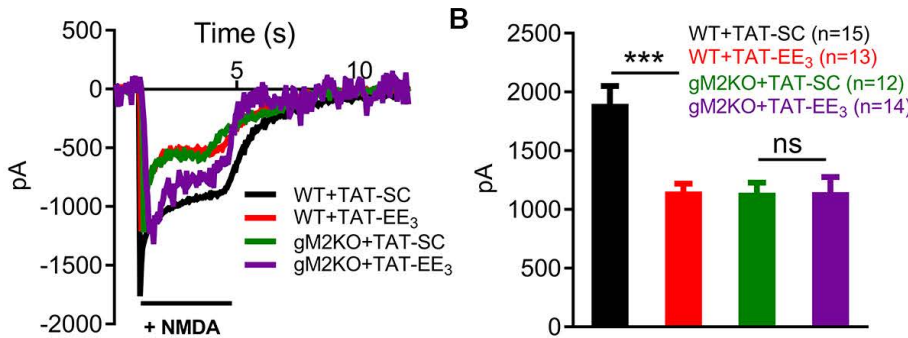

D
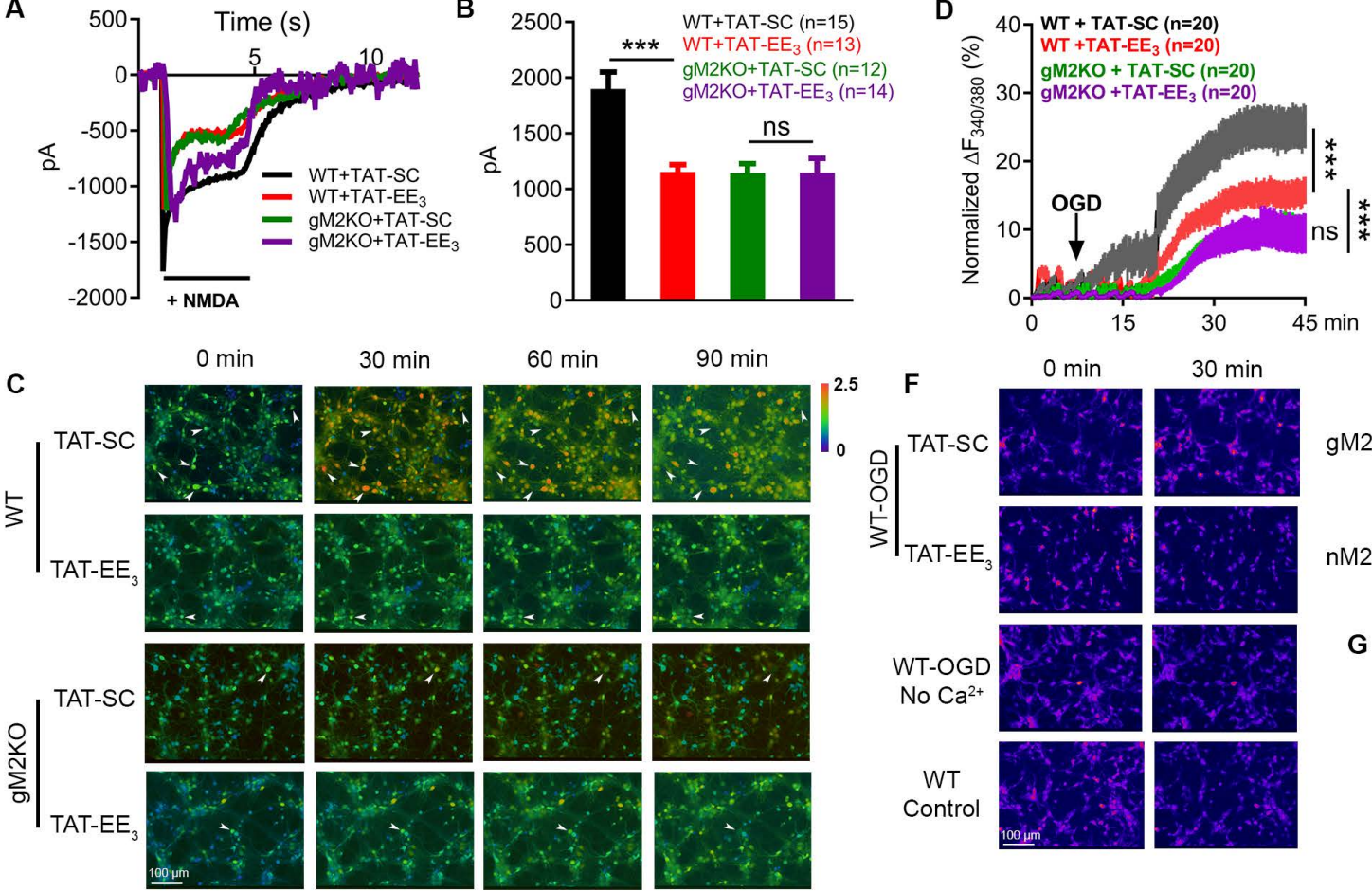

$30 \min$
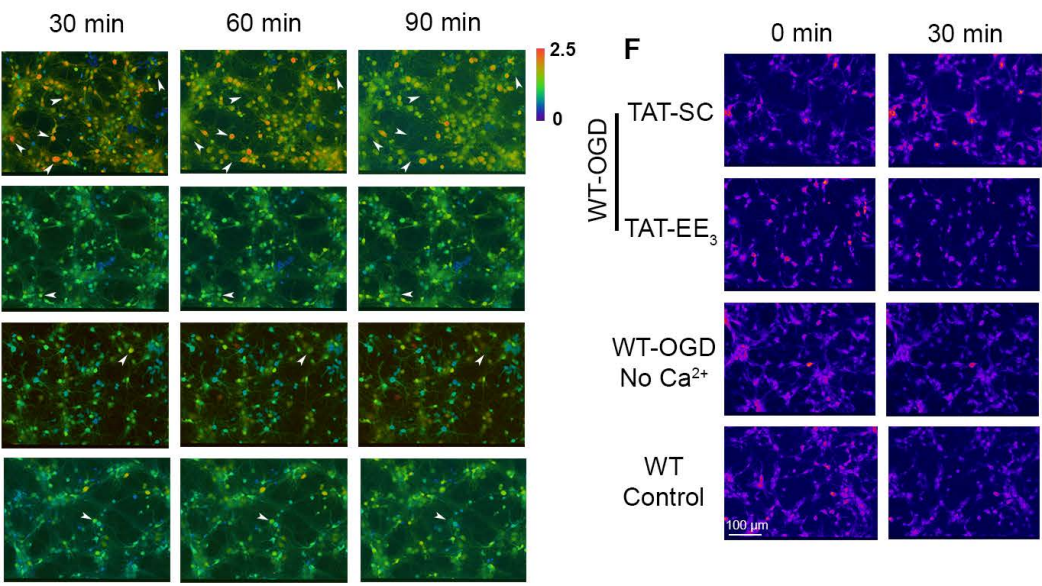

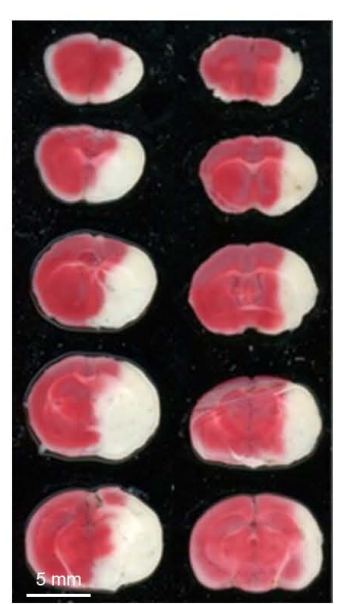

TAT-SC TAT-EE
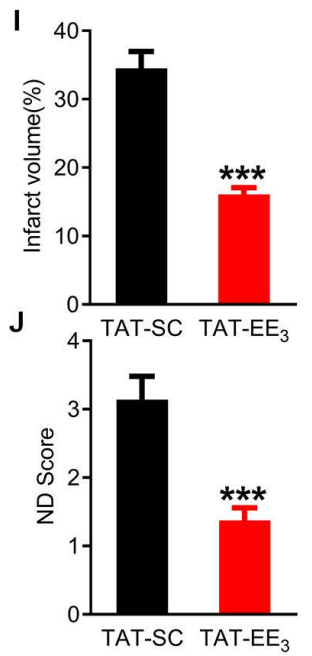

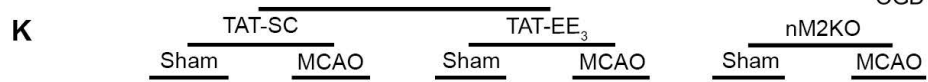

WT
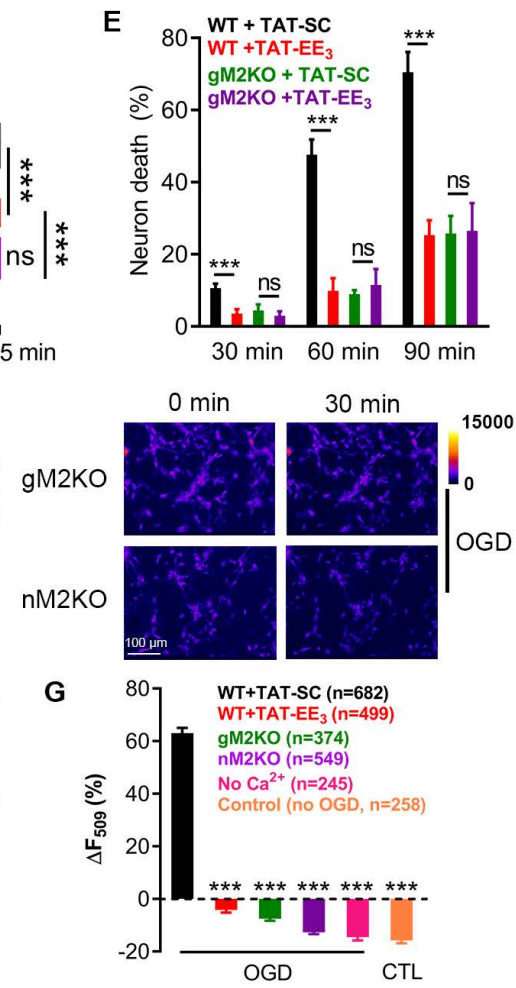

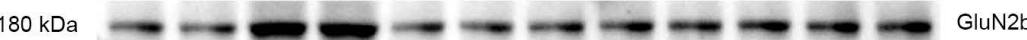

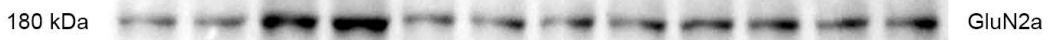

$120 \mathrm{kDa}=--m-m-m-m-m$ GluN1a

$130 \mathrm{kDa} C \mathrm{C}$
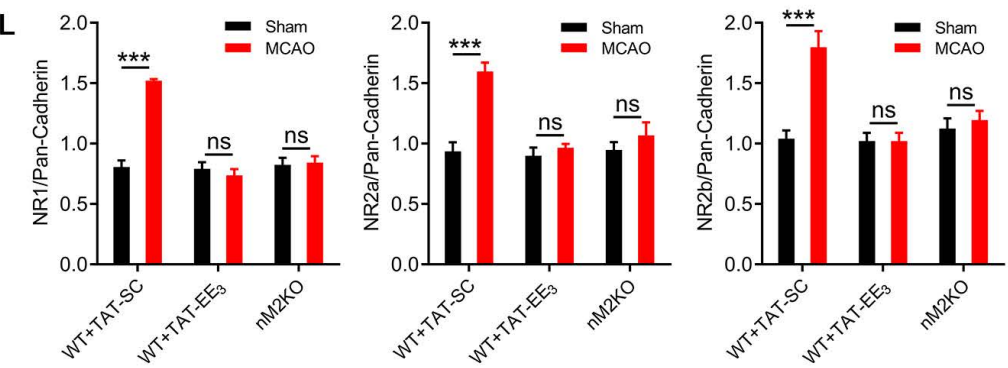

WT

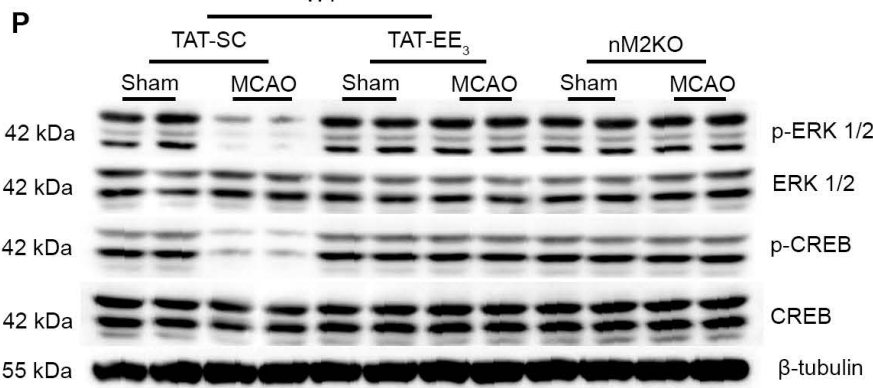

N

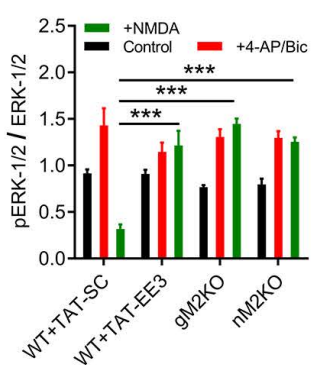

O

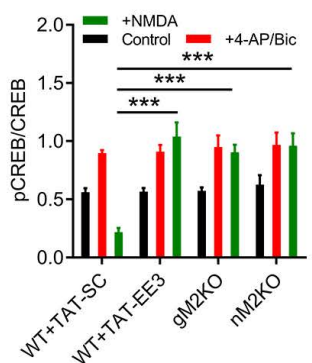

Q

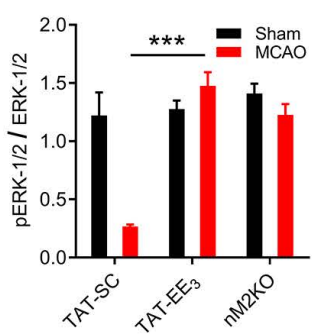

$\mathbf{R}$

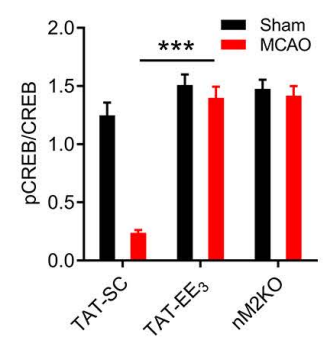


A
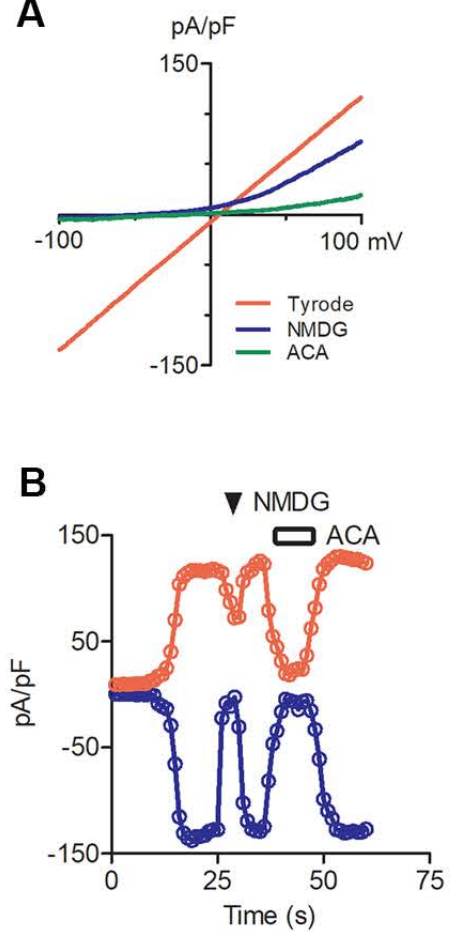

C

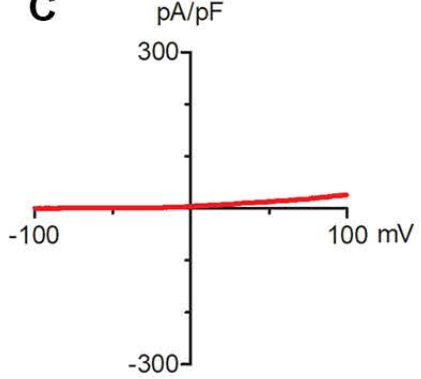

D

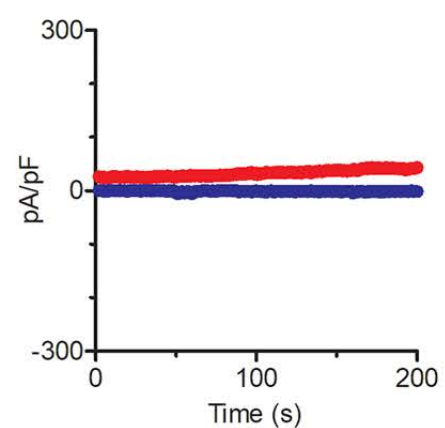

E

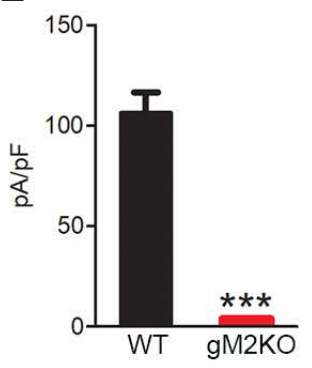

H

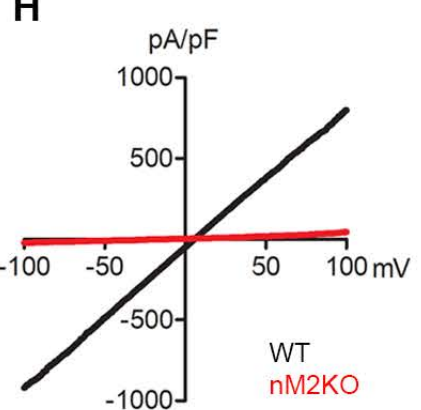

Supplementary figure 1

I

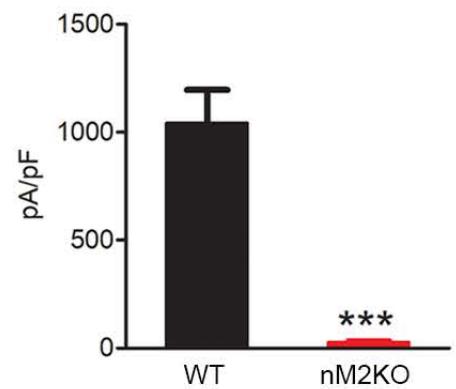

F

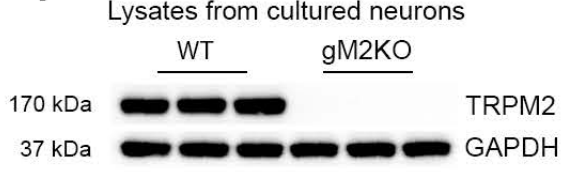

G

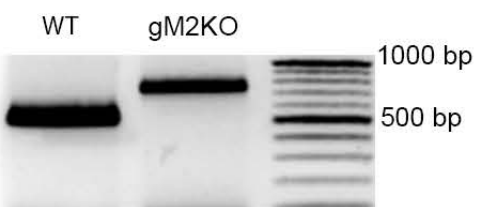

J Lysates from cultured neurons

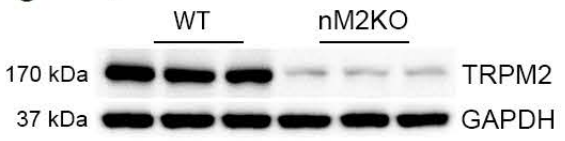

K

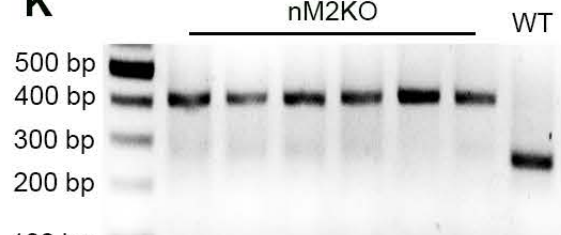

$100 \mathrm{bp}$ 


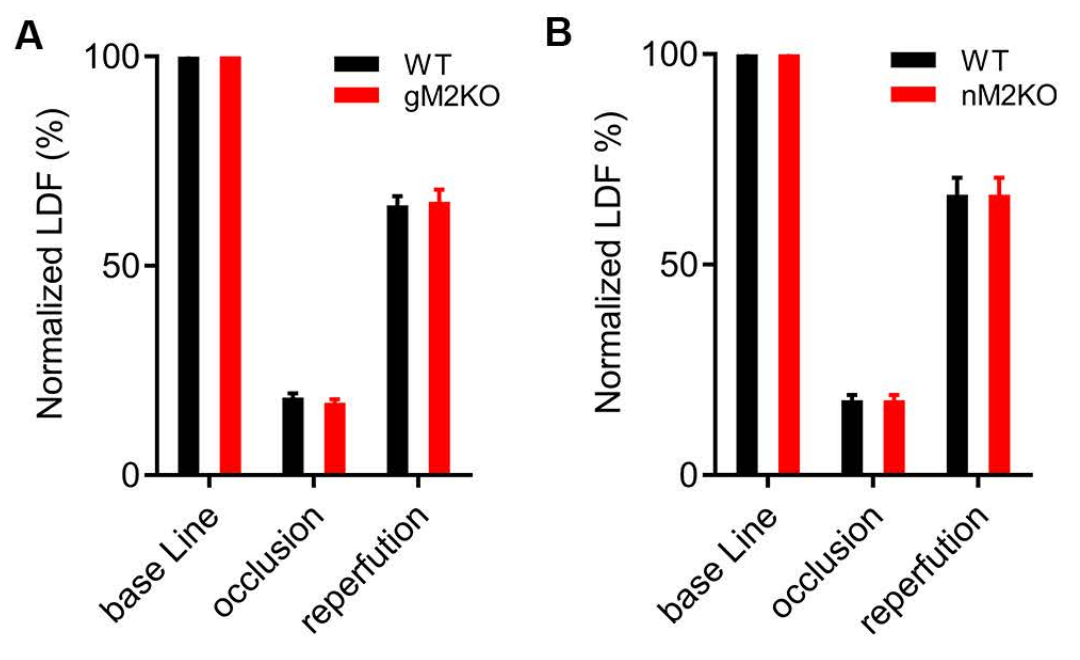

Supplementary figure 2 

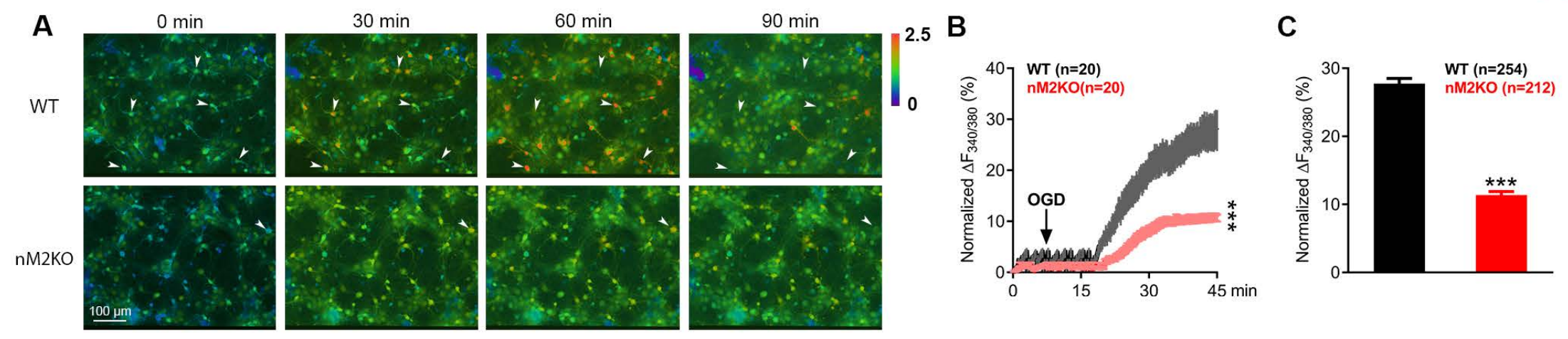

Supplementary figure 3

D

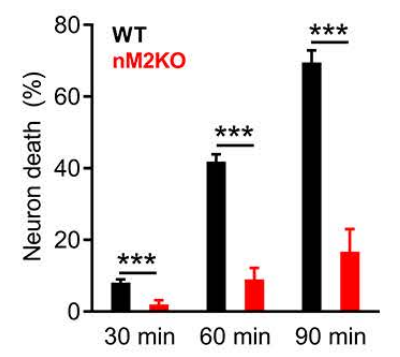



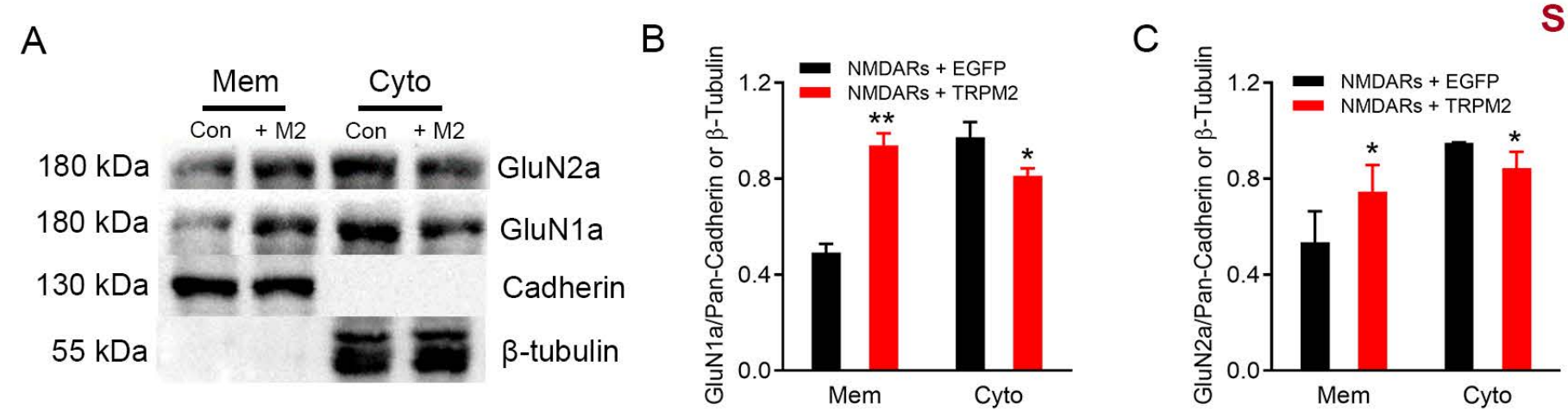

Supplementary figure 4

D

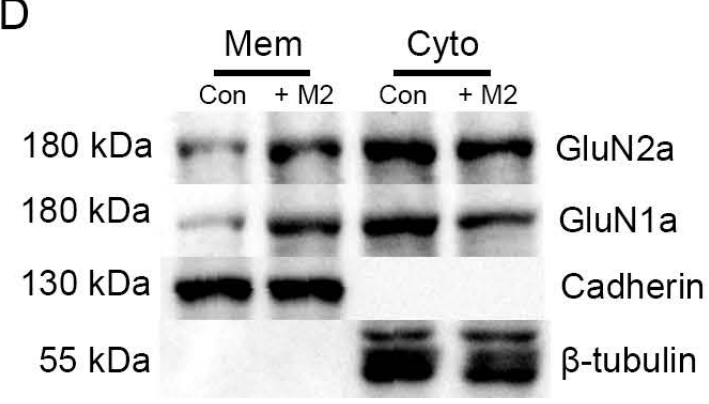

E

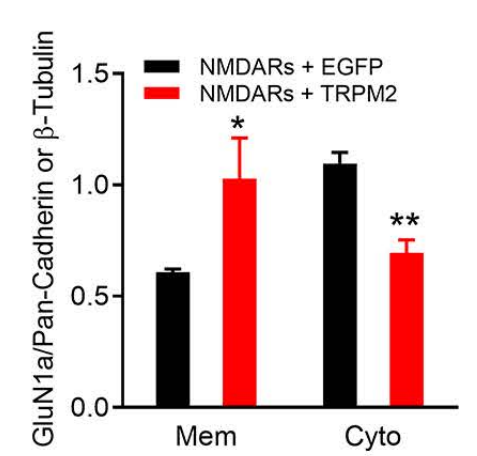

F

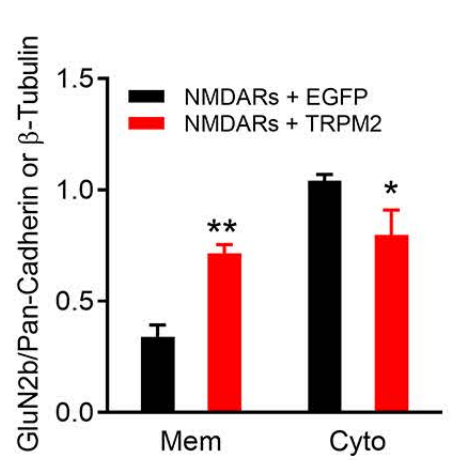

G

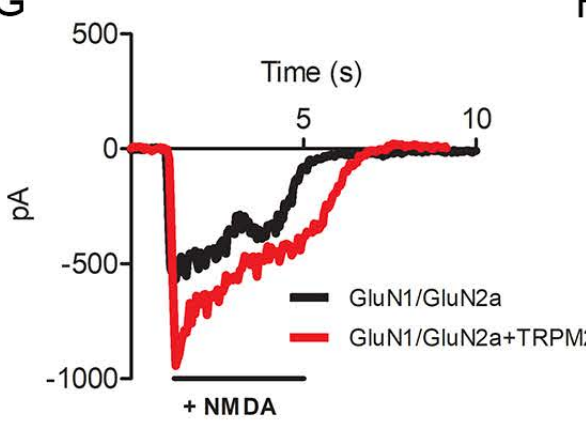

I

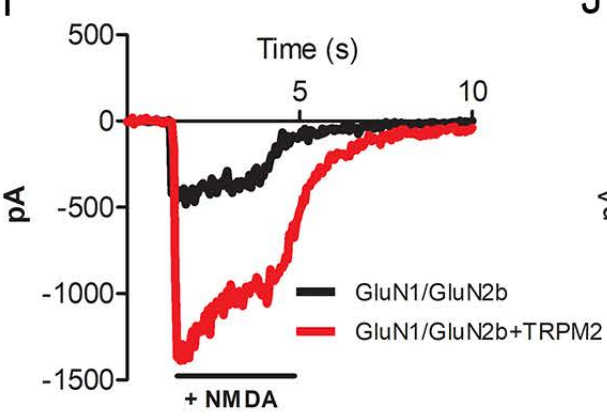

$\mathrm{H}$

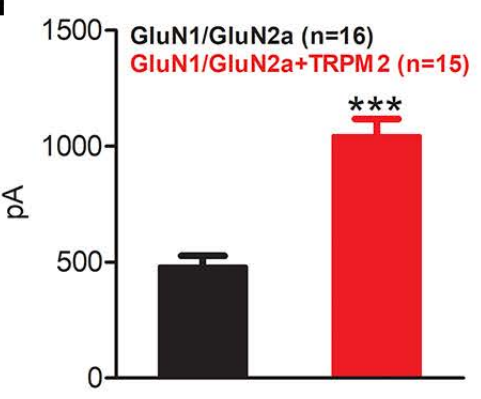

J

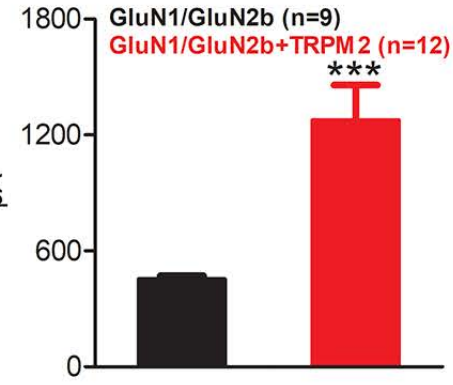


A

(1) 653

695

hTRPM1 (1) LVACKLYKAMAHESSESDLVDDISQDLDNNSRDEGLLAELLD

hTRPM2 (1) -ACSKILKELSKEEE---DTDSSEEMLALA EEYEHRAIGVET

hTRPM3 (1) -VACKLCKAMAHEASENDMVDDISQEINHNSRDEGQLAVELID

hTRPM4 (1) -GACLLLRVMARLEE---DAEEAARRKDLAFKEEGMGVDLFG

hTRPM5 (1) -AACKILKEMSHLET-----EAEAARATREAKYERLALDLES

hTRPM6 (1) -IACILYRAMAHEAKES HMVDDAS EELKNYSKKOFGQLALDLLE

hTRPM7 (1) -VACKIYRSMAYEAK QSDLVDDTSEEIKQYSNDE EQLAVELLE

hTRPM8 (1) - GASKLLKTLAKVKN--- DINAAGESEELANEYETRAVELET

Consensus (1) LVACKLYKAMAHESSES DLVDDISQDIDNNSKDEGQLALELLD

B
H. sapiens
(1) 653
695
M. musculus
(1) -ACSKILKELSKE--EEDTDSSEEMLALAEEYEHRAIGVET
A. carolinensis
(1) -ACSKILKELSKE--EEDTDSSEEMLALADEFEHRAIGVET
G. gallus
(1) -ACSKILKELSKE--ETDTDSTEEMLALAEEYEHRAIGVET
$X$. laevis
(1) -ACSKILKELAKE--EEDTDTTDEMLALAEQYEHKAIGVET
D. rerio (1) --ASKILRKLAOESGEDDSEEATEMLELANHYEKQAIGVES
(1) -ACSKLLKELSKE--EEDTDTSEEMLALAEEFEQRAIGIFT
D. melanogaster (1) NTCAHIAAMQGSVKV IEELMKEDRSGVISARNKLTDA---Consensus (1) ACSKILKELSKE EEDTDSSEEMLALAEEYEHRAIGVET
C

D

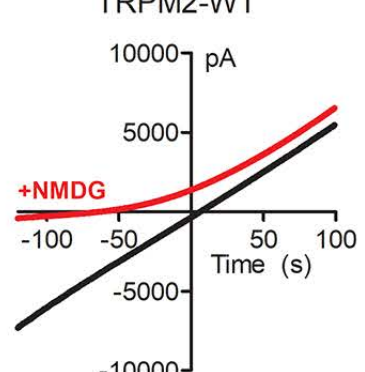

$\mathbf{F}$

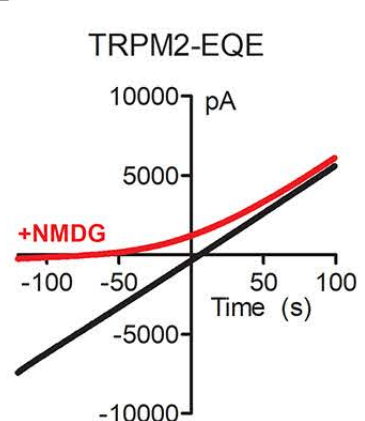

G
Supplementary figure 5

E

TRPM2- $\triangle \mathrm{EE}_{3}$

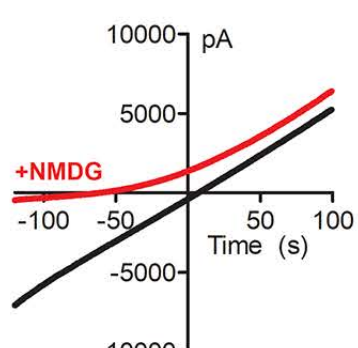

$-10000$

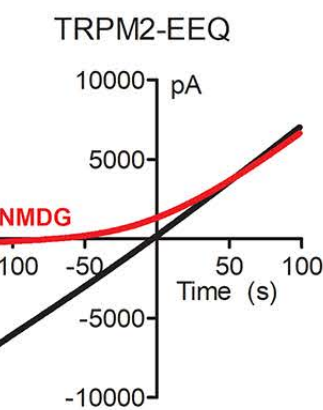

TRPM2-QEE

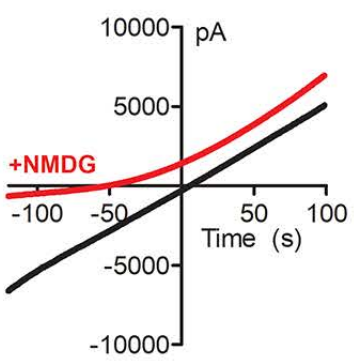

H

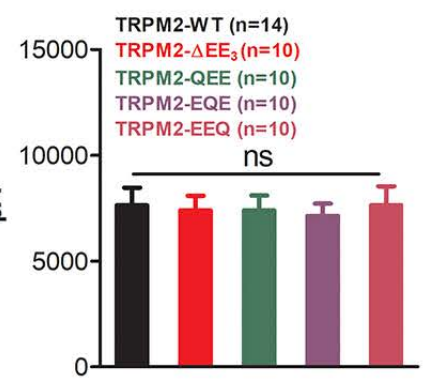



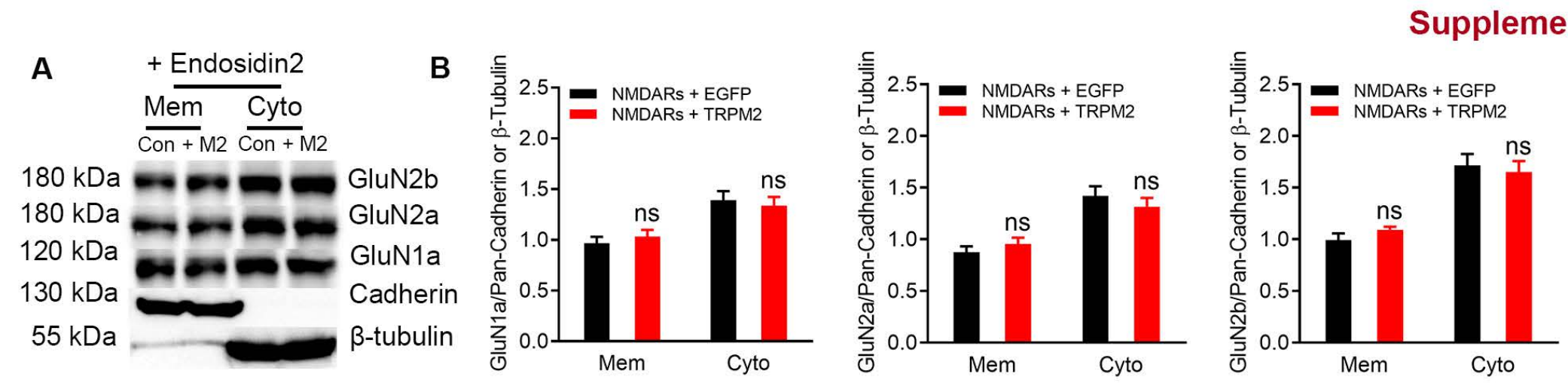


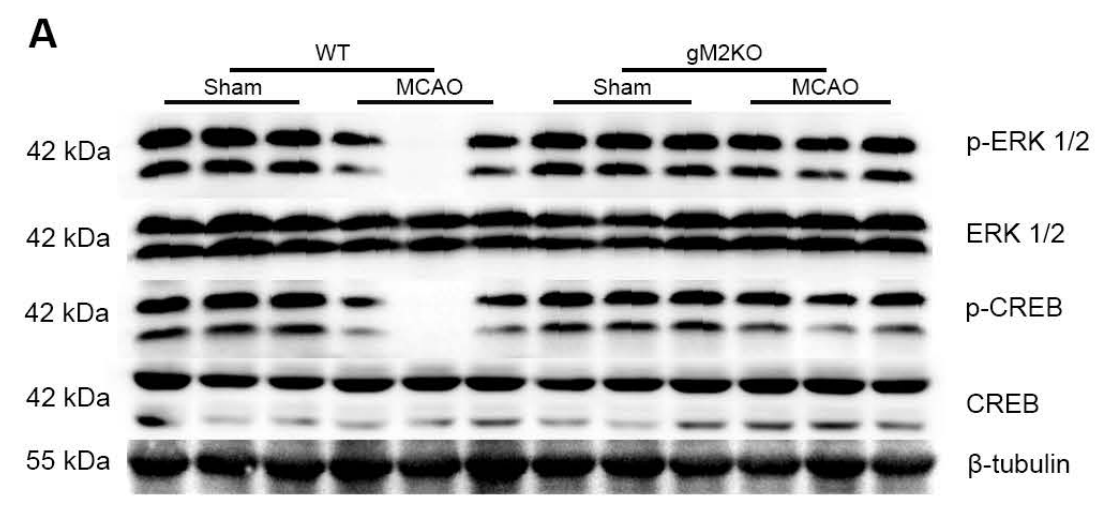

B

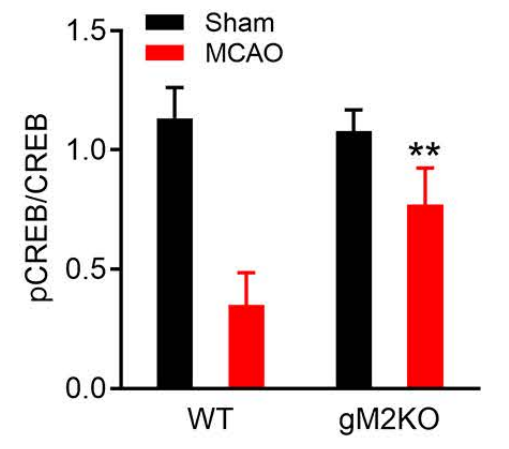

C

Supplementary figure 7

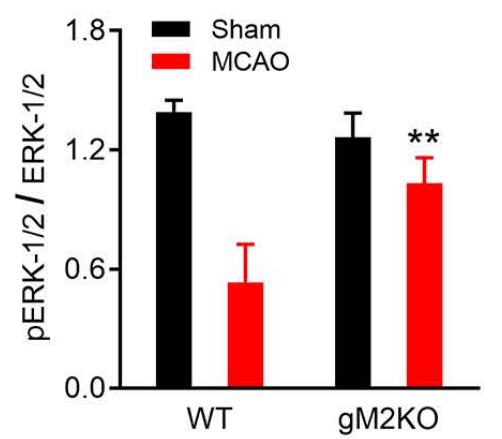

